Igor Mancilla Lourenço

Estudo numérico da dinâmica de um conjunto de tubos ejetando fluido sob instabilidade paramétrica

São Paulo 
Igor Mancilla Lourenço

\section{Estudo numérico da dinâmica de um conjunto de tubos ejetando fluido sob instabilidade paramétrica}

Versão Corrigida

Dissertação apresentada à Escola Politécnica da Universidade de São Paulo para obtenção do título de Mestre em Ciências.

Área de concentração: Engenharia de estruturas

Orientador: Prof. Dr. Guilherme Rosa Franzini 
Autorizo a reprodução e divulgação total ou parcial deste trabalho, por qualquer meio convencional ou eletrônico, para fins de estudo e pesquisa, desde que citada a fonte.

Este exemplar foi revisado e corrigido em relação à versão original, sob responsabilidade única do autor e com a anuência de seu orientador.

São Paulo, de de

Assinatura do autor:

Assinatura do orientador:

\section{Catalogação-na-publicação}

Lourenço, Igor

Estudo numérico da dinâmica de um conjunto de tubos ejetando fluido sob instabilidade paramétrica / I. Lourenço -- versão corr. -- São Paulo, 2020. $94 \mathrm{p}$.

Dissertação (Mestrado) - Escola Politécnica da Universidade de São Paulo. Departamento de Engenharia de Estruturas e Geotécnica.

1.Dinâmica não linear 2.Instabilidade paramétrica 3.Vibração induzida pelo escoamento interno 4.Teoria de Floquet I.Universidade de São Paulo. Escola Politécnica. Departamento de Engenharia de Estruturas e Geotécnica II.t. 


\section{Agradecimentos}

Ao longo desta jornada tive a honra de conhecer e trabalhar com grandes profissionais. Alguns contribuíram de forma direta na criação e desenvolvimento de cada assunto estudado no presente trabalho. Todo processo criativo e intelectual está diretamente conectado com o ambiente ao qual estamos inseridos.

Agradeço a Escola Politécnica da Universidade de São Paulo, corpo técnico, professores, funcionários dos setores administrativos e de limpeza por oferecerem todas as necessidades básicas, intelectuais e de infraestrutura para o bom desenvolvimento de estudos e pesquisas.

Levo junto comigo, por todo este caminho que devo percorrer, um carinho especial pelas pessoas que fizeram parte da rotina de pesquisa desenvolvida junto ao laboratório de mecânica offshore LMO. Agradeço a todos os colegas de trabalho com quem aprendi muito mais que teorias: Renato Finotelli, Giovanni Amaral, João Cerqueira, Michel Miúdo, Fernando Toni e Guilherme Vernizzi. Apesar de não fazer parte do grupo do LMO não poderia deixar de mencionar meu grande amigo Breno Ayres Pereira Mendes.

Deixo registrada a minha admiração por todos os professores com quem convivi durante o desenvolvimento desta pesquisa. Dentre todos, manifesto meu mais profundo respeito citando aqueles que realizaram alguma contribuição importante neste trabalho: Prof. Celso P. Pesce, Prof. Renato M. M. Orsino e Prof. Carlos E. N. Mazzilli.

Agradeço ao meu orientador Prof. Guilherme Rosa Franzini por todo apoio, tempo e dedicação oferecidos a mim e ao meu trabalho ao longo destes anos. Um grande exemplo de caráter, honestidade e ética. Me ensinou que o processo de aprendizado não se limita aos capítulos dos livros, sempre deixando bem claro em nos mostrar toda a bagagem que já havíamos adquirido ao longo do tempo. Muito Obrigado por tudo.

Tudo isto não teria sido possível sem o apoio daqueles que acreditaram e torceram pelo meu sucesso ao longo destes anos. Agradeço a minha família por terem tido paciência e por terem servido como suporte nos momentos mais difíceis. Agradeço principalmente aos meus pais: Valdemar Lourenço Filho e Sandra R. M. Lourenço, sem a ajuda deles nenhuma destas palavras teriam sido marcadas nas folhas de papel. 
"A inovação não é só a criação do inédito, mas também a revitalização do antigo."

(Mário Sergio Cortella, 2019) 


\section{Resumo}

Os risers são estruturas comumente empregadas na engenharia oceânica e que têm como uma de suas funções o transporte de fluidos. Essas estruturas estão sujeitas, dentre outros esforços a solicitações decorrentes de excitação paramétrica e do escoamento interno. No caso em estudo, a excitação paramétrica ocorre quando o termo de rigidez da equação de movimento varia de maneira explícita com o tempo. No caso dos risers, essa variação está associada à modulação da força normal decorrente do movimento da plataforma no plano vertical. O foco da pesquisa aqui descrita é analisar um modelo simplificado de um riser formado por um conjunto de dois tubos rígidos conectados por molas rotacionais e que ejeta fluido a partir da extremidade livre. Por sua vez, a excitação paramétrica é obtida pela aplicação de movimentos verticais harmônicos ao suporte. O estudo do problema combinado foi conduzido por meio da Teoria de Floquet para o problema linearizado, possibilitando a determinação das regiões de estabilidade e instabilidade da solução trivial do sistema não autônomo. O problema combinado estudado a partir das equações não lineares foi analisado por meio de mapas de amplitude de respostas pós-crítica construídos numericamente. Dentre outros resultados, mostra-se que a variação da velocidade do escoamento interno, frequência e amplitude do movimento imposto interferem de forma significativa nos diagramas de instabilidade e nos mapas de amplitude pós-crítica, os quais apresentam significativa erosão no plano de parâmetros estudado.

Palavras-chave: Vibração induzida pelo escoamento interno, instabilidade paramétrica, Teoria de Floquet, dinâmica não linear 


\section{Abstract}

Risers are structures commonly found in the offshore engineering scenario. These structures are responsible, among other functions, for conveying fluid. Risers are subjected to several dynamic phenomena, particularly those related to parametric instability and to internal flow. In this study, parametric instability occurs when the stiffness parameter of the equation of motion explicitly depends on time. In the risers' scenario, such a variation is associated with the normal forces modulation caused by the motions of the floating units on the vertical plane. The focus of this research is to analyze a simplified model of riser composed of two rigid pipes, connected by rotational springs and ejecting fluid from its free end. Parametric instability arises from vertical and harmonic motions applied to the support. Herein, the study of the linearized problem is carried out by means of the Floquet Theory. This approach allowed identifying, in the plane of parameters that govern the problem, stability and instability regions of the trivial solution. In addition to this linear analysis, maps showing the post-critical response as a function of some control parameters are obtained using the non-linear mathematical model. Among other findings, the present dissertation shows that the presence of internal flow significantly affects the stability of the trivial solution. Furthermore, the concomitant parametric excitation and internal flow effects led to maps of post-critical response with a marked erosion.

Keywords: Internal-flow-induced vibration, parametric instability, Floquet Theory, nonlinear dynamics 


\section{Lista de ilustrações}

Figura 1 - Exemplo de risers em situação de operação. . . . . . . . . . . . . . . 18

Figura 2 - Níveis de ordem hierárquica. . . . . . . . . . . . . . . . 19

Figura 3 - Exemplo de aplicação do SWIR . . . . . . . . . . . . . . . . . . 21

Figura 4 - Modelo dos tubos rígidos submetidos a escoamento axial interno proposto por Benjamin. . . . . . . . . . . . . . . . . . 23

Figura 5 - Modelo de placas paralelas para tubos de parede fina. . . . . . . . . . . 24

Figura 6 - Modelo do tubo engastado-livre proposto. . . . . . . . . . . . . 25

Figura 7 - Comportamento do sistema com e sem escoamento pulsante. . . . . . . 26

Figura 8 - Mapa de bifurcação. . . . . . . . . . . . . . . . . . 27

Figura 9 - Tubo contínuo com escoamento interno apoiado em suportes elásticos. . 28

Figura 10 - Diagrama de Strutt. . . . . . . . . . . . . . . . . . . . . . 30

Figura 11 - Mapas de amplitude dos deslocamentos laterais . . . . . . . . . . . . 32

Figura 12 - Comparação dos espaços de fase da solução quase estática para diferentes amplitudes de excitação paramétrica com frequência adimensional $n=2,0.33$

Figura 13 - Modelo do problema clássico de Benjamin conectado a um reservatório a jusante. . . . . . . . . . . . . . . . . . . 35

Figura 14 - Resultado da variação de amplitude do ciclo limite em função do parâmetro do adimensional de massa $\gamma$. . . . . . . . . . . . . . . . 36

Figura 15 - Modelo do cabo flexível vertical com uma extremidade fixada na plataforma e a outra no leito marinho. . . . . . . . . . . . . . . . . . . . . 37

Figura 16 - Resultados a partir da análise via Teoria de Floquet. . . . . . . . . . . 38

Figura 17 - Diagrama de Strutt para equação de Mathieu. . . . . . . . . . . . . . . 42

Figura 18 - Modelo estudado. . . . . . . . . . . . . . . . . . . . . . 44

Figura 19 - Volume de controle. . . . . . . . . . . . . . . . . . . . 45

Figura 20 - Forças de pressão. . . . . . . . . . . . . . . . . . . . 47

Figura 21 - Vetores $\mathbf{r}_{0}$ e $\mathbf{r}_{L} \ldots \ldots \ldots \ldots \ldots \ldots$

Figura 22 - Modelo 2 graus de liberdade. . . . . . . . . . . . . . . . 52

Figura 23 - Diagrama de estabilidade para $\beta=0,200$. Os eixos das abcissas e das

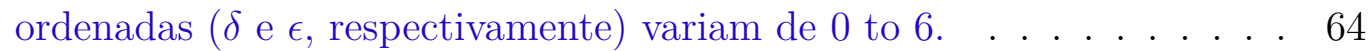

Figura 24 - Diagrama de estabilidade para $\beta=0,500$. Os eixos das abcissas e das ordenadas ( $\delta$ e $\epsilon$, respectivamente) variam de 0 to $6 . \ldots 65$

Figura 25 - Diagrama de estabilidade para $\beta=0,750$. Os eixos das abcissas e das ordenadas $(\delta$ e $\epsilon$, respectivamente) variam de 0 to $6 . \ldots 66$

Figura 26 - Diagramas de estabilidade para o caso $\beta=0,750 \ldots$. . . . . . . . 68

Figura 27 - Diagramas de estabilidade para o caso $\beta=0,750$. . . . . . . . . . 69

Figura 28 - Diagramas de estabilidade para o caso $\beta=0,500 \ldots \ldots$. . . . . . . 70 
Figura 29 - Respostas das séries temporais dos ângulos $\theta_{1}$ e $\theta_{2}$ para $u=13,4 ; \zeta=5 \%$ e $\beta=0,500 \ldots \ldots \ldots \ldots \ldots 71$

Figura 30 - Mapa de amplitude e estatística da resposta não linear $y_{(\tau)} \cdot u=0,0$, $\zeta=0 \%$ e $\beta=0,750 \ldots \ldots \ldots \ldots . \ldots \ldots 74 \ldots \ldots \ldots$

Figura 31 - Mapa de amplitude e estatística da resposta não linear $y_{(\tau)} \cdot u=12,0$, $\zeta=0 \%$ e $\beta=0,750 \ldots \ldots \ldots \ldots \ldots$

Figura 32 - Respostas das séries temporais de $y_{t}(\tau)$ para $u=12,0, \zeta=0 \%, \beta=$ 0,750 em dois pontos no plano de parâmetros $(\delta ; \epsilon) \ldots \ldots 76$

Figura 33 - Mapa de amplitude e estatística da resposta não linear $y_{(\tau)} \cdot u=12,0$,

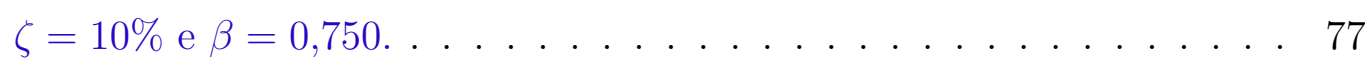

Figura 34 - Mapa de amplitude e estatística da resposta não linear $y_{(\tau)} \cdot u=13,4$, $\zeta=5 \%$ e $\beta=0,500 \ldots \ldots \ldots \ldots 79 \ldots \ldots \ldots$

Figura 35 - Respostas das séries temporais de $y_{t}(\tau)$ para $u=13,4, \zeta=5 \%, \beta=$

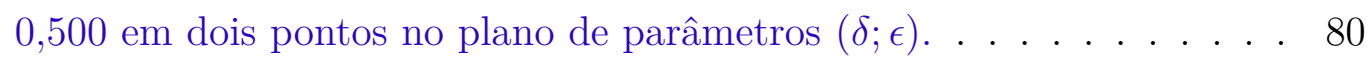

Figura 36 - Mapa de amplitude e estatística da resposta não linear $y_{(\tau)} \cdot u=16,0$, $\zeta=0 \%$ e $\beta=0,750 \ldots \ldots \ldots$. . . . . . . . . . 80

Figura 37 - Respostas das séries temporais de $y_{t}(\tau)$ para $u=16,0, \zeta=0 \%, \beta=$ 0,750 em quatro pontos no plano de parâmetros parameters $(\delta ; \epsilon)$. . . 82

Figura 38 - Modelo 1 grau de liberdade. . . . . . . . . . . . . . . . . . . . . . 89

Figura 39 - Obtenção do vetor posição $\mathbf{r}_{L} \ldots \ldots$. . . . . . . . . . . . . . . . . . . 91

Figura 40 - Diagrama de instabilidade para o modelo de 1 grau de liberdade considerando $u=0$ e $K_{1}=0 \ldots \ldots$. . . . . . . . . . . . . . . . . . . 93

Figura 41 - Diagrama de Strutt para equação de Mathieu . . . . . . . . . . . . . . 94 


\section{Lista de tabelas}

Tabela 1 - Tubo escolhido para o cálculo dos coeficientes de amortecimento e rigidez. 50

Tabela 2 - Parâmetros adimensionais e os valores adotados para $\beta=0,200$. . . 58

Tabela 3 - Parâmetros adimensionais e os valores adotados para $\beta=0,500$. . . . 58

Tabela 4 - Parâmetros adimensionais e os valores adotados para $\beta=0,750$. . . . 59

Tabela 5 - Valores de velocidade crítica para diferentes $\beta \ldots \ldots . \ldots . \ldots$ 


\section{Lista de abreviaturas e siglas}

EDO Equação Diferencial Ordinária

LMO Laboratório de Mecânica Offshore

PPGEC Programa de Pós-Graduação em Engenharia Civil

SWIR Sea Water Intake Riser

TLP Tension Leg Platform 


\section{Lista de símbolos}

A Matriz de monodromia para a solução da equação de Mathieu utilizando a teoria de Floquet.

$A$

$a_{0}$

$a_{1}$

$B_{0}$

$B_{c}$

C

$\mathbf{C}^{*}$

$\overline{\mathrm{C}}$

$\overline{\mathbf{C}}^{*}$

$C_{i, j}^{s}$

$\mathbf{C}_{\text {est }}$

$c_{i, j}^{s}$

$D$

$d$

$E_{0}$
Amplitude do movimento imposto ao suporte

Coeficiente de amortecimento proporcional de massa utilizado para determinação da matriz de amortecimento proporcional

Coeficiente de amortecimento proporcional de rigidez utilizado para determinação da matriz de amortecimento proporcional

Área superfície aberta do volume de controle

Área superfície fechada do volume de controle

Matriz de amortecimento linearizada para o problema do pêndulo duplo considerando os tubos ejetando fluido em com movimento imposto no suporte

Matriz de amortecimento linearizada adimensional para o problema do pêndulo duplo considerando os tubos ejetando fluido em com movimento imposto no suporte

Matriz de amortecimento não linear para o problema do pêndulo duplo considerando os tubos ejetando fluido em com movimento imposto no suporte

Matriz de amortecimento não linear adimensional para o problema do pêndulo duplo considerando os tubos ejetando fluido em com movimento imposto no suporte

Termos adimensionais da matriz de amortecimento

Matriz de amortecimento estrutural proporcional

Termos da matriz de amortecimento adimensional

Diâmetro externo do tubo

Diâmetro interno do tubo

Módulo de elasticidade do material do tubo 


\begin{tabular}{|c|c|}
\hline$E$ & Energia potencial total \\
\hline $\mathbf{F}$ & Vetor de forças externas para a equação não linear de movimento \\
\hline $\mathbf{F}^{*}$ & $\begin{array}{l}\text { Vetor de forças externas adimensional para a equação não linear de } \\
\text { movimento }\end{array}$ \\
\hline$f_{i}$ & $\begin{array}{l}\text { Valor da i-ésima frequência natural para o problema do tubo contínuo } \\
\text { posicionado na horizontal sem escoamento interno }\end{array}$ \\
\hline$g$ & Valor numérico do módulo da aceleração da gravidade \\
\hline$I$ & Momento de inércia da seção transversal do tubo \\
\hline K & $\begin{array}{l}\text { Matriz de rigidez linearizada para o problema do pêndulo duplo con- } \\
\text { siderando os tubos ejetando fluido em com movimento imposto no } \\
\text { suporte }\end{array}$ \\
\hline $\mathbf{K}^{*}$ & $\begin{array}{l}\text { Matriz de rigidez linearizada adimensional para o problema do pêndulo } \\
\text { duplo considerando os tubos ejetando fluido em com movimento imposto } \\
\text { no suporte }\end{array}$ \\
\hline$\overline{\mathbf{K}}$ & $\begin{array}{l}\text { Matriz de rigidez não linear para o problema do pêndulo duplo con- } \\
\text { siderando os tubos ejetando fluido em com movimento imposto no } \\
\text { suporte }\end{array}$ \\
\hline$\overline{\mathbf{K}}^{*}$ & $\begin{array}{l}\text { Matriz de rigidez não linear adimensional para o problema do pêndulo } \\
\text { duplo considerando os tubos ejetando fluido em com movimento imposto } \\
\text { no suporte }\end{array}$ \\
\hline$\hat{\mathbf{K}}$ & $\begin{array}{l}\text { Matriz de rigidez linearizada para o problema do pêndulo duplo conside- } \\
\text { rando os tubos preenchidos por fluido mas sem ejeção e sem movimento } \\
\text { vertical imposto no suporte. }\end{array}$ \\
\hline$K_{i}$ & $\begin{array}{l}\text { Adimensional de rigidez flexional equivalente das molas do conjunto de } \\
\text { tubos }\end{array}$ \\
\hline$k_{i}$ & Rigidez flexional equivalente do i-ésimo tubo \\
\hline$L$ & Lagrangeano \\
\hline$L_{i}$ & Comprimento da i-ésimo tubo \\
\hline$\hat{L}$ & Comprimento total do conjunto de tubos \\
\hline M & $\begin{array}{l}\text { Matriz de massa linearizada para o problema do pêndulo duplo con- } \\
\text { siderando os tubos ejetando fluido em com movimento imposto no } \\
\text { suporte }\end{array}$ \\
\hline
\end{tabular}


M* Matriz de massa linearizada adimensional para o problema do pêndulo duplo considerando os tubos ejetando fluido em com movimento imposto no suporte

$\overline{\mathbf{M}} \quad$ Matriz de massa não linear para o problema do pêndulo duplo considerando os tubos ejetando fluido em com movimento imposto no suporte

$\overline{\mathbf{M}}^{*} \quad$ Matriz de massa não linear adimensional para o problema do pêndulo duplo considerando os tubos ejetando fluido em com movimento imposto no suporte

$\hat{\mathbf{M}} \quad$ Matriz de massa linearizada para o problema do pêndulo duplo considerando os tubos preenchidos por fluido mas sem ejeção e sem movimento vertical imposto no suporte.

M Massa por unidade de comprimento do fluido interno ao tubo

$m$

$\mathbf{n}$

$\mathbf{n}_{L}$
Massa por unidade de comprimento do tubo

Soma das massa do tubo e do fluido, ambas por unidade de comprimento Versor unitário normal às seções de entrada e saída do fluido no sistema de controle

Versor unitário normal à seção de saída do fluido (extremidade inferior do conjunto de tubos)

Pressão exercida pelo fluido no interior do tubo na i-ésima superfície

Funções periódicas auxiliares

Posição da extremidade superior do tubo

Posição da extremidade inferior do tubo

Área superfície aberta do sistema de controle

Área superfície fechada do sistema de controle

Área da seção transversal do tubo

Área definida pela i-ésima superfície de controle

Energia cinética total

Tempo 
Velocidade do escoamento interno em relação a um referencial solidário ao movimento do tubo

Velocidade do escoamento interno adimensionalizada

Velocidade do escoamento interno crítica

Velocidade do fluido em relação a um referencial fixo

Velocidade do volume de controle

Função periódica que caracteriza a variação da posição do suporte no tempo

Posição vertical da extremidade livre do conjunto de tubos ao longo do tempo adimensional

Valor da média de oscilação da série temporal $y_{t}(\tau)$

Valor do desvio padrão da série temporal $y_{t}(\tau)$

Adimensional de massa

Adimensional da frequência do movimento imposto ao suporte

Variação da energia potencial do sistema aberto de controle no instante inicial

Deslocamento virtual

Variação da energia cinética do sistema aberto de controle no instante inicial

Trabalho virtual das forças não conservativas

Adimensional da amplitude do movimento imposto ao suporte

Taxa de amortecimento viscoso proporcional de Rayleigh, associado ao i-ésimo modo de vibrar do conjunto de tubos preenchido por fluido mas sem ejeção

Ângulo formado pelo eixo do i-ésimo tubo e a direção vertical

Adimensional de comprimento do segundo trecho do tubo

Constante que define o valor da frequência natural para o problema do tubo contínuo obtido em Blevins (1984) 


$\begin{array}{ll}\hat{\lambda}_{n} & \begin{array}{l}\text { n-ésimo autovalor definido pela análise de estabilidade da teoria de } \\ \text { Floquet }\end{array} \\ \lambda^{*} & \text { Valor numérico do maior autovalor, em módulo, definido para análise } \\ & \text { de estabilidade via teoria de Floquet } \\ \rho & \text { Massa específica do fluido } \\ \tau & \text { Tempo adimensional } \\ \bar{\tau} & \text { Período adimensional } \\ \Phi & \text { Matrix de monodromia obtida pela analise de estabilidade através da } \\ & \text { teoria de Floquet para o problema do pêndulo duplo sujeito a escoamento } \\ & \text { interno e instabilidade paramétrica } \\ & \text { Razão entre os valores de rigidez flexional e geométrica do sistema } \\ \omega & \text { Frequência do movimento imposto ao suporte } \\ \hat{\omega}_{i} & \text { i-ésima frequência natural }\end{array}$




\section{Sumário}

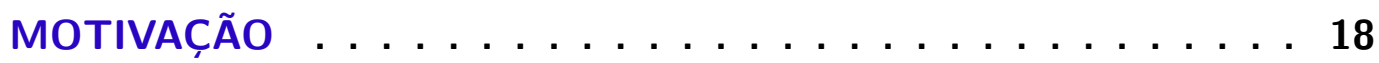

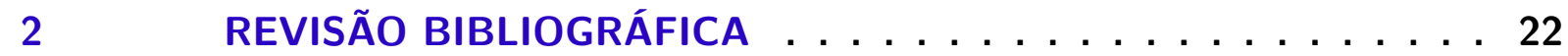

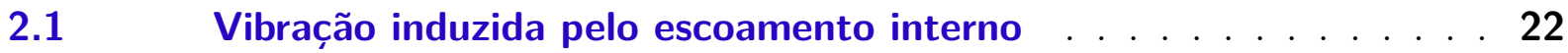

2.2 Excitação paramétrica . . . . . . . . . . . . . . 28

2.3 Efeitos combinados de escoamento interno e excitação paramétrica 34

2.4 Teoria de Floquet . . . . . . . . . . . . . . . . . . . . 38

2.4.1 Exemplo de aplicação à equação de Mathieu . . . . . . . . . . . . . . 40

$2.5 \quad$ Lacunas de conhecimento . . . . . . . . . . . . . . . 42

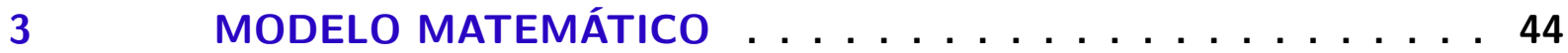

3.1 Trabalho virtual das forças não conservativas . . . . . . . . 45

3.2 Parâmetros de rigidez e amortecimento . . . . . . . . . . . 48

3.2.1 Determinação dos coeficientes de rigidez . . . . . . . . . . . . . . . . 49

3.2.2 Determinação dos coeficientes de amortecimento . . . . . . . . . . . 50

$3.3 \quad$ Modelo 2 graus de liberdade . . . . . . . . . . . . . . 51

4 METODOLOGIA $\ldots \ldots \ldots \ldots$

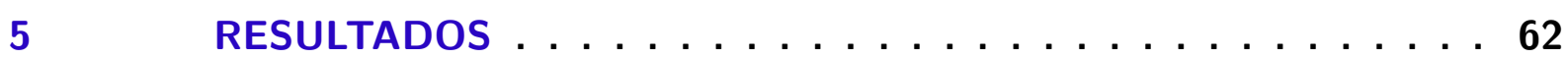

$5.1 \quad$ Análise Linear . . . . . . . . . . . . . . . . . . . 62

$5.2 \quad$ Análise Não Linear . . . . . . . . . . . . . . . . . . . . . 72

$6 \quad$ CONCLUSÃO $\ldots \ldots \ldots \ldots$

Referências . . . . . . . . . . . . . 85

A MODELO 1 GRAU DE LIBERDADE . . . . . . . . . . 89

A.1 Determinação das equações de movimento . . . . . . . . . . . . 89

A.2 Resultados . . . . . . . . . . . . . . . . 93 


\section{Resumo e organização textual}

O objetivo deste trabalho é realizar o estudo numérico acerca da resposta dinâmica de um conjunto de dois tubos rígidos conectados entre si por molas rotacionais lineares e submetidos a efeitos combinados de vibração induzida pelo escoamento interno e excitação paramétrica. O modelo proposto é formado por um conjunto de barras rígidas articulado na extremidade superior e livre na extremidade inferior. Amortecedores concentrados são empregados para modelar a dissipação estrutural.

No Capítulo 1 trará a contextualização a respeito das motivações que levaram ao desenvolvimento do presente estudo. O Capítulo 2 apresentará a revisão bibliográfica, onde serão expostos os principais trabalhos que servirão como referência para concepção deste, permitindo também identificar lacunas que serão preenchidas com o auxílio do presente trabalho.

Após a revisão bibliográfica, o Capítulo 3 apresentará as hipóteses e premissas adotadas para a formulação das equações de movimento não lineares e linearizadas para o problema com dois graus de liberdade. No Capítulo 4 serão discutidas as metodologias utilizadas para a resolução das equações diferenciais não lineares e linearizadas do problema proposto.

O Capítulo 5 exibirá os diagramas de estabilidade e os mapas de resposta pós-crítica obtidos para os problemas linearizado e não linear, além de toda a discussão a respeito das conclusões obtidas a partir da análise dos resultados. Por fim, no Capítulo 6, serão discutidas as perspectivas de trabalhos futuros e tecidas as conclusões obtidas. 


\section{Motivação}

O trabalho aqui documentado consiste do estudo numérico com um modelo de baixa hierarquia definido por um conjunto de tubos sujeitos a instabilidade paramétrica e escoamento interno. O problema motivador e que serve como pano de fundo para a pesquisa é, por certo, mais complexo e encontra-se relacionado à dinâmica de risers. Os risers são estruturas esbeltas usadas em engenharia oceânica e que estão sujeitas a carregamentos decorrentes dos escoamentos externo e interno, além de movimentos aplicados ao seu topo decorrentes da movimentação da unidade flutuante ao qual eles estão conectados. Em particular, o movimento da unidade flutuante leva a uma variação temporal da força normal, induzindo assim o problema de excitação paramétrica ${ }^{1}$

Figura 1 - Exemplo de risers em situação de operação.

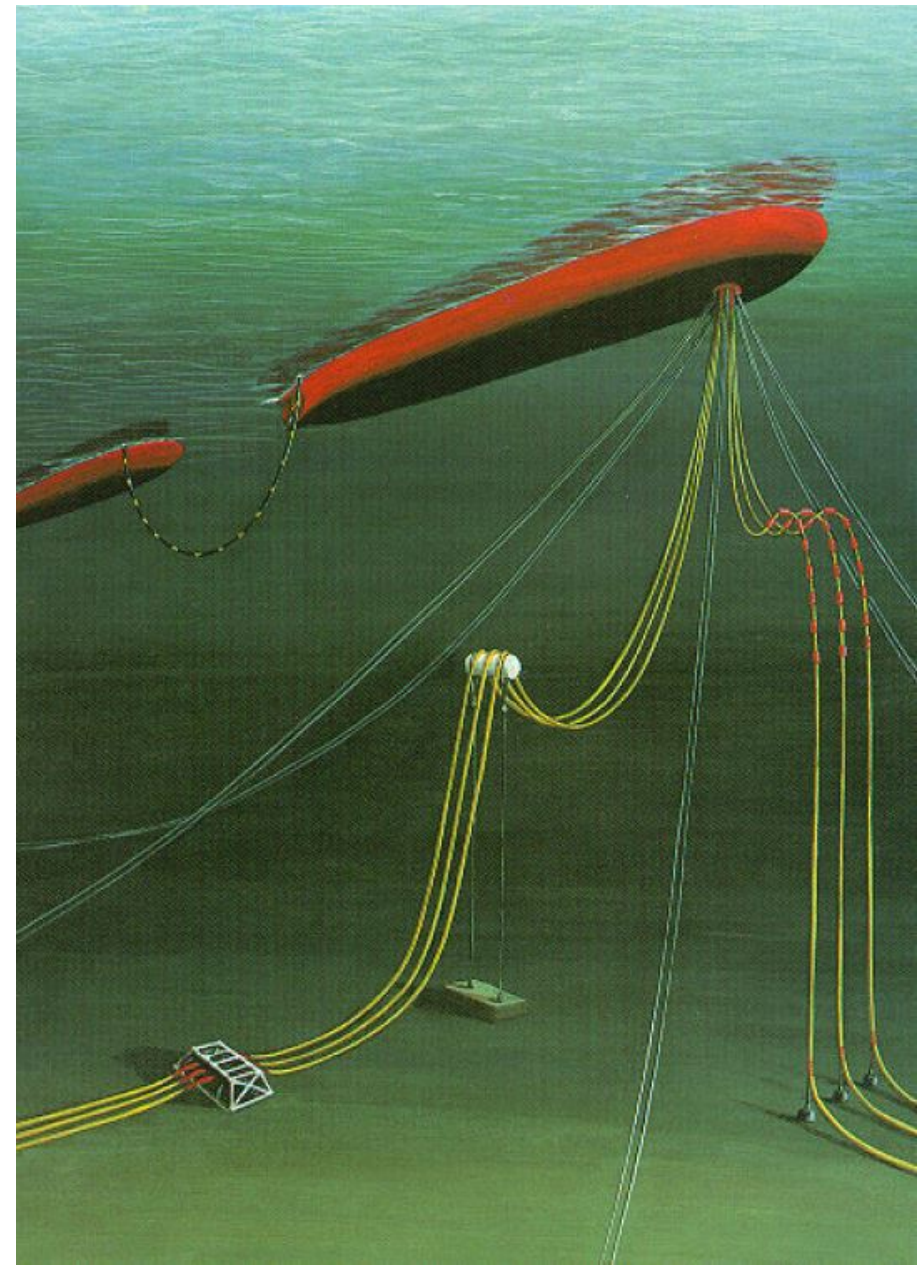

Fonte: Pesce (1997).

$\overline{1}$ Mais detalhes a respeito do problema de excitação paramétrica serão mostrados na Seção 2.2. 
Na Figura 1 é possível observar diversos risers respeitando diferentes configurações de lançamento. A Figura 1 também auxilia no entendimento dos fenômenos dinâmicos aos quais essas estruturas estão suscetíveis. Esses tubos estão submersos e os efeitos de escoamento externo podem desencadear o fenômeno de vibrações induzidas pela emissão de vórtices $^{2}$ (VIV). Este texto não entrará em maiores detalhes sobre o VIV por não ser objeto de estudo do presente trabalho. No entanto, o leitor interessado em um aprofundamento no tema é indicado a consultar o livro-texto Blevins (2001).

Para grandes profundidades, os risers possuem uma de suas extremidades fixa em plataformas flutuantes que, por sua vez, sofrem influência dos esforços de primeira e segunda ordem oriundos das ondas de superfície. Os risers de extração possuem fluidos escoando em seu interior, sendo esse escoamento de sucção (ou aspiração) ou de ejeção. O trabalho aqui desenvolvido avalia os efeitos do problema de escoamento interno de ejeção. Mais detalhes a respeito dos efeitos do escoamento interno em um tubo serão vistos na Seção 2.1.

Por se tratar de um problema de grande complexidade, para possibilitar um melhor entendimento do comportamento da estrutura submetida a efeitos combinados, muitas vezes as primeiras análises são realizadas em modelos de menor nível hierárquico. Na Figura 2 são propostos modelos de diferentes hierarquias que pretendem avaliar com, um certo nível de aceitação, o comportamento dinâmico de um tubo conduzindo fluido.

Figura 2 - Níveis de ordem hierárquica.

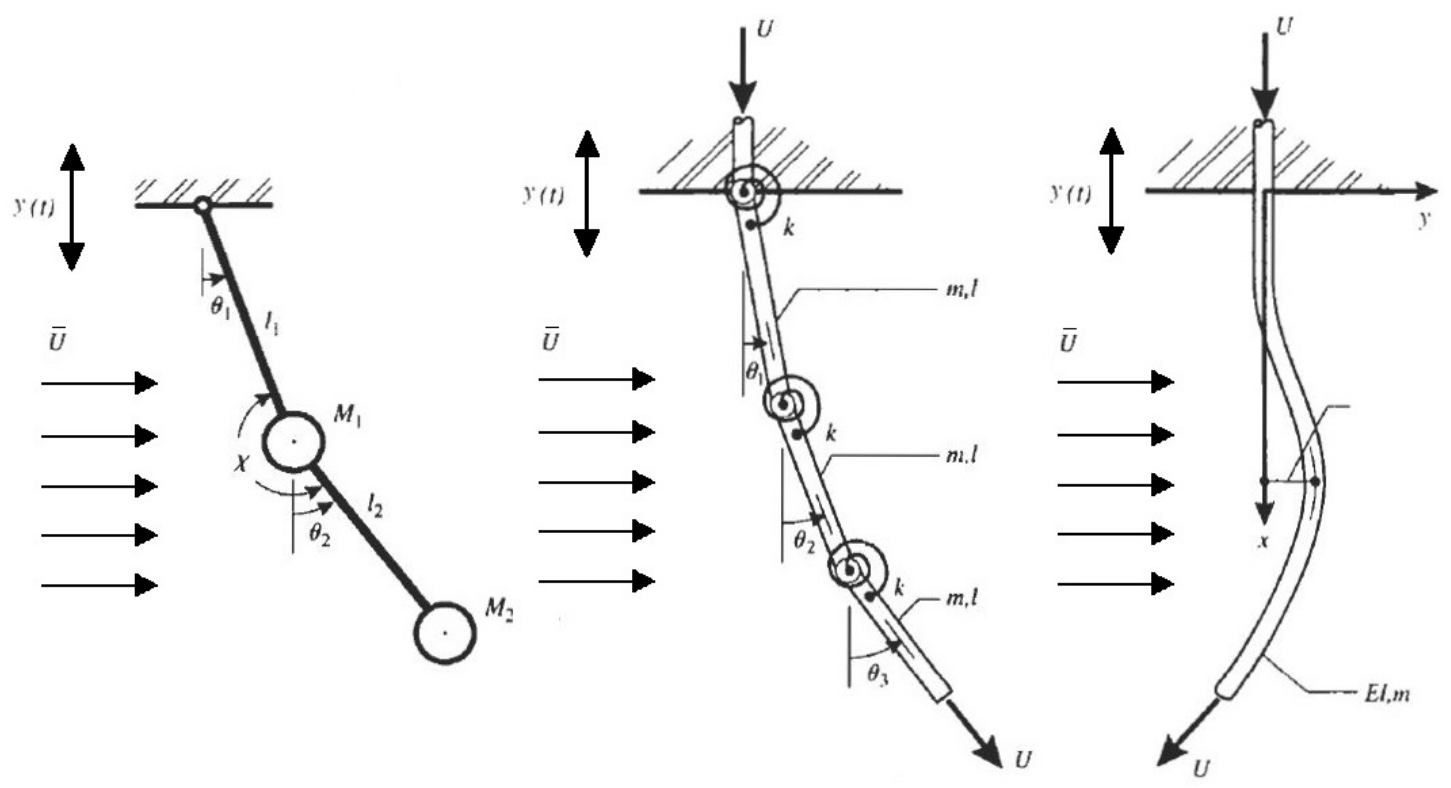

Fonte: Extraída de Païdoussis (1998).

$\overline{2}$ O VIV é um fenômeno auto-excitado que ocorre em corpos submersos em campos de escoamento de fluidos viscosos, sendo ocasionado pelo desprendimento alternado de vórtices. 
Os modelos propostos na Figura 2 estão organizados do modelo com menor hierarquia (à esquerda) até o com maior hierarquia (ver figura mais à direita). O nível de complexidade da solução vai aumentando de acordo com o nível hierárquico estudado. O segundo modelo apresentado já possibilita a avaliação da concomitância de todos os efeitos, mas por se tratar de barras rígidas os resultados podem ser diferentes dos obtidos pelo modelo de maior hierarquia, representado por tubo flexível.

Visando o ganho de conhecimento para posterior estudo em um modelo de maior hierarquia, esta dissertação foca em um problema simplificado, no qual dois tubos rígidos são conectados entre si por molas e amortecedores. Esse conjunto de tubos é submetido tanto a escoamento interno de ejeção quanto ao movimento vertical imposto no suporte, sendo o último, o responsável pela indução da excitação paramétrica. O objetivo aqui é mostrar como a dinâmica do conjunto é enriquecida pela concomitância entre os problemas de vibrações induzidas pelo escoamento interno de ejeção e de excitação paramétrica.

Embora as concepções usuais de risers envolvam vinculações nas duas extremidades, existe uma nova tecnologia que vem sendo estudada denominada SWIR (sea water intake riser). Um SWIR é uma tubulação fixa nas plataformas com um lastro em sua extremidade inferior e possui a função de captar água de grandes profundidades para diversas utilizações na plataforma como, por exemplo, controlar a temperatura de equipamentos. Este trabalho tem como uma de suas motivações o estudo de um SWIR, embora apenas os casos de ejeção de fluido e movimentos da plataforma sejam objetos da dissertação. Na Figura 3 é mostrado o SWIR em fase de operação.

Existe ainda grande necessidade da realização de estudos para o desenvolvimento de métodos e tecnologias para extração de petróleo e também coleta e devolução de água em grandes profundidades. No entanto e tendo em vista a complexidade desses problemas, é interessante o estudo em problemas simplificados e com foco em poucos efeitos considerados de maneira simultânea. Espera-se que tais estudos possam auxiliar no esclarecimento do comportamento dos problemas tecnológicos e de maior complexidade. 
Figura 3 - Exemplo de aplicação do SWIR

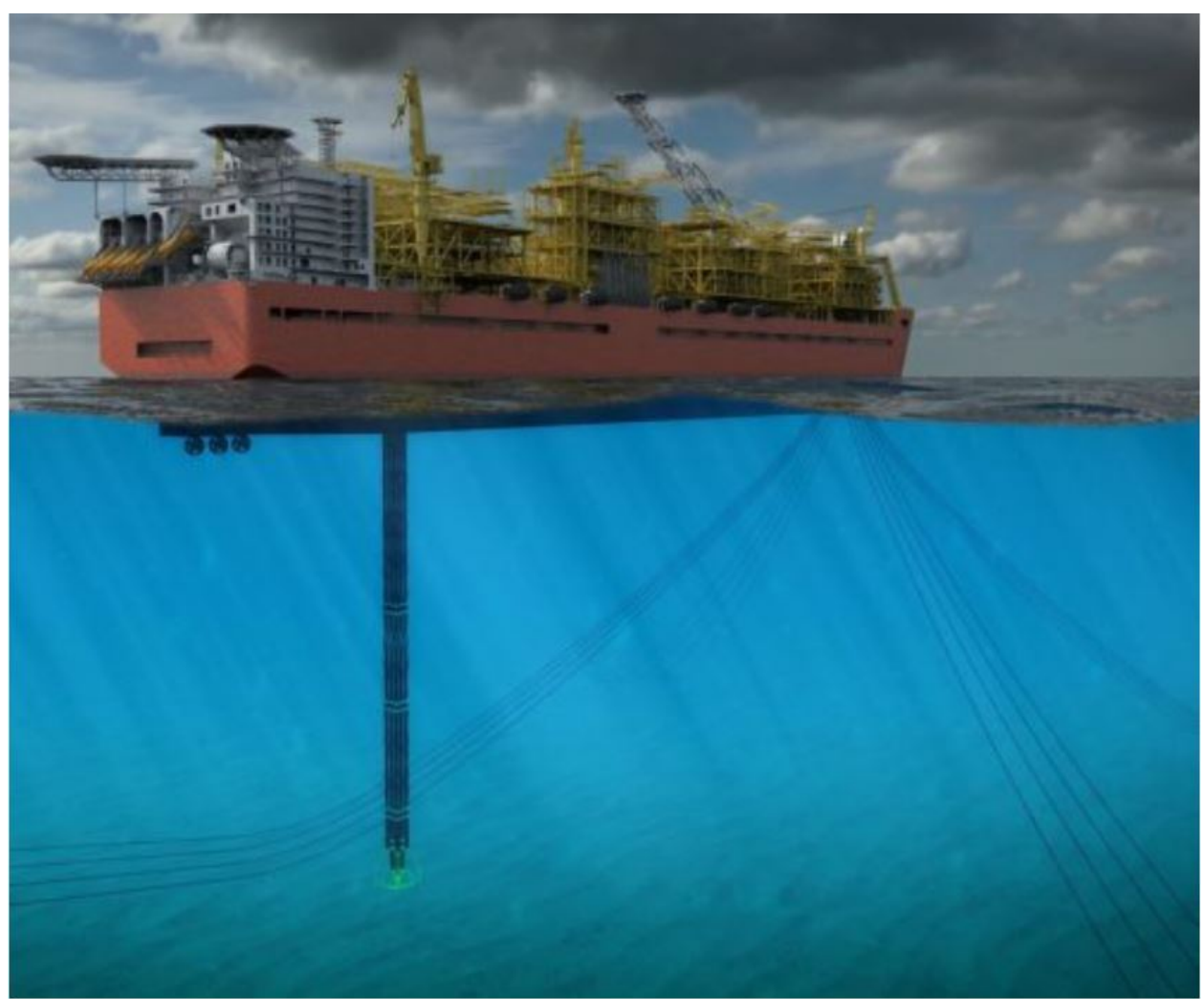

Fonte: www.sealengineering.fr 


\section{Revisão Bibliográfica}

Neste capítulo serão apresentados trabalhos desenvolvidos em cada área de pesquisa na qual o presente trabalho está inserido. Serão apresentados artigos e teses em diferentes assuntos dentro de cada área de conhecimento, de modo a mostrar as diferentes possibilidades de pesquisa dentro de cada uma delas. Cada assunto será tratado separadamente, dentre eles o problema do escoamento interno em tubos, excitação paramétrica, efeitos combinados e teoria de Floquet. Cada um destes assuntos podem ser observados nas Seções 2.1, 2.2, 2.3 e 2.4 respectivamente.

\subsection{Vibração induzida pelo escoamento interno}

O estudo do problema de vibração induzida pelo escoamento axial em estruturas esbeltas vem sendo feito desde meados da década de 50 do século XX. Um dos autores pioneiros no assunto foi Thomas Brooke Benjamin, que publicou uma sequência com dois trabalhos. No primeiro deles, Benjamin (1961a), foi proposta a equação de movimento de um tubo engastado livre com escoamento interno, considerando a velocidade de escoamento constante no tempo. Ainda nesse trabalho, Benjamin empregou o principio estendido de Hamilton para encontrar as equações de movimento para o problema tratado como meio contínuo, além de realizar uma análise de estabilidade para um modelo de dois tubos rígidos articulados (ver Figura 4).

Como resultado da análise de estabilidade do sistema com dois graus de liberdade, o autor propôs um gráfico que relaciona as parcelas reais e imaginárias dos autovalores para diversos valores de velocidade de escoamento interno, explicitando as regiões de estabilidade e instabilidade da configuração inicial. As equações de movimento linearizadas descritas em Benjamin (1961a) podem ser observadas nas Equações 2.1 e 2.2, onde $M$ e $m$ são as massas por unidade de comprimento do fluido e do tubo, $L_{1}$ e $L_{2}$ são os comprimentos do primeiro e do segundo tubo, $k_{1}$ e $k_{2}$ representam as molas rotacionais que conectam os tubos, $\theta_{1}$ e $\theta_{2}$ são os ângulos formados pelo eixo de cada tubo com a direção a vertical, $U$ é a velocidade do escoamento interno e $g$ é o valor da aceleração gravitacional.

$$
\begin{aligned}
& (M+m)\left[\left(\frac{1}{3} L_{1}^{3}+L_{1}^{2} L_{2}\right) \ddot{\theta}_{1}+\frac{1}{2} L_{1} L_{2}^{2} \ddot{\theta}_{2}\right]+M U\left[L_{1}^{2} \dot{\theta}_{1}+2 L_{1} L_{2} \dot{\theta}_{2}-L_{1} U\left(\theta_{1}-\theta_{2}\right)\right]+ \\
& +k_{1} \theta_{1}+k_{2}\left(\theta_{1}-\theta_{2}\right)+\frac{1}{2}(M+m) g\left(L_{1}^{2}+2 L_{1} L_{2}\right) \theta_{1}=0 \\
& (M+m)\left(\frac{1}{2} L_{1} L_{2}^{2} \ddot{\theta}_{1}+\frac{1}{3} L_{2}^{3} \ddot{\theta}_{2}\right)+M U L_{2}^{2} \dot{\theta}_{2}+k_{2}\left(\theta_{2}-\theta_{1}\right)+\frac{1}{2}(M+m) g L_{2}^{2} \theta_{2}=0
\end{aligned}
$$


Figura 4 - Modelo dos tubos rígidos submetidos a escoamento axial interno proposto por Benjamin.

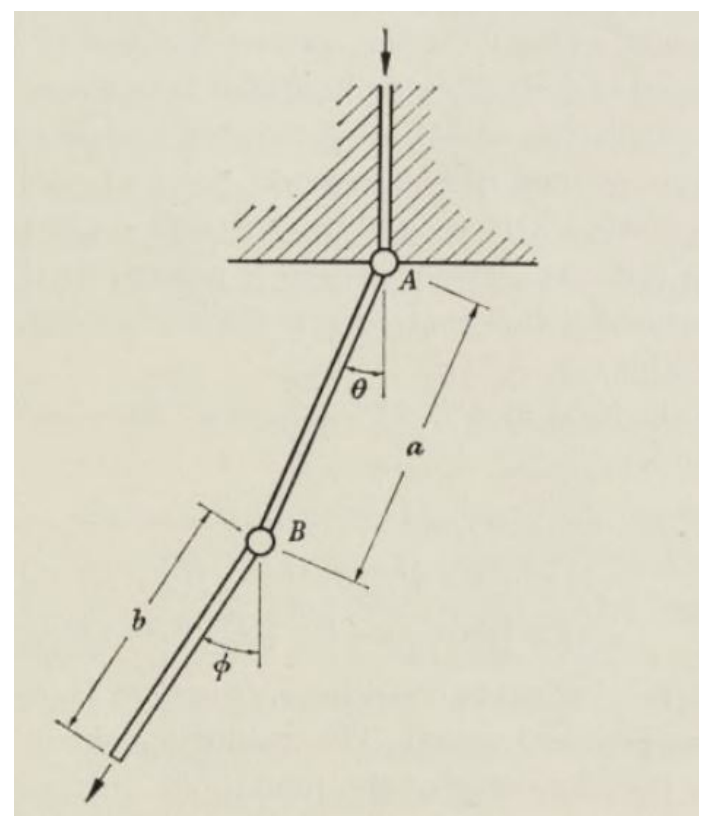

Fonte: Benjamin (1961a).

No segundo trabalho, Benjamin (1961b), foi realizado um estudo experimental para validar os resultados obtidos no primeiro artigo da série. No novo trabalho, o autor observou a ocorrência de 3 formas de instabilidade. Ocorreu flambagem nos experimentos em que o conjunto de tubos estavam vinculados segundo condições de contorno engastado-livre e engastado-apoiado. Além da flambagem foi observado também instabilidade dinâmica, ocorrendo quando a velocidade de escoamento interno ultrapassa um determinado valor crítico, levando a oscilações auto-excitadas.

Em Gregory e Païdoussis (1966a) foi encontrada a equação de movimento para o tubo contínuo através da segunda lei de Newton, além da solução analítica das frequências naturais do sistema contínuo. Os autores ainda propuseram diagramas que verificam a estabilidade dos modos desejados como função da velocidade de escoamento interno. No trabalho são propostas análises para diferentes valores de $\beta$, definido como a relação entre a massa do fluido e a massa total do sistema, ambas por unidade de comprimento do tubo.

Em Gregory e Païdoussis (1966b) foi feita a análise experimental para validar os resultados apresentados no artigo citado anteriormente. A análise experimental mostrou que o amortecimento, associado à capacidade de dissipação de energia do material do tubo ou à transferência de energia ocasionada pelo escoamento, em certos casos pode estabilizar o sistema. Ao comparar os resultados da análise experimental com o modelo matemático deduzido em Gregory e Païdoussis (1966a) foi possível comprovar a validação do modelo.

No trabalho desenvolvido por Weaver e Paidoussis (1977) foi estudado o problema 
de estabilidade em tubos de parede fina com escoamento interno. Dois modelos teóricos simplificados que representam tubos de parede fina foram desenvolvidos a partir de placas lisas posicionadas paralelamente. O primeiro modelo foi desenvolvido respeitando os critérios de ondas estacionárias e placas com largura e comprimento finitos, enquanto no segundo modelo foram consideradas ondas progressivas, comprimento infinito e largura finita. O modelo esquemático do problema pode ser observado na Figura 5.

Figura 5 - Modelo de placas paralelas para tubos de parede fina.

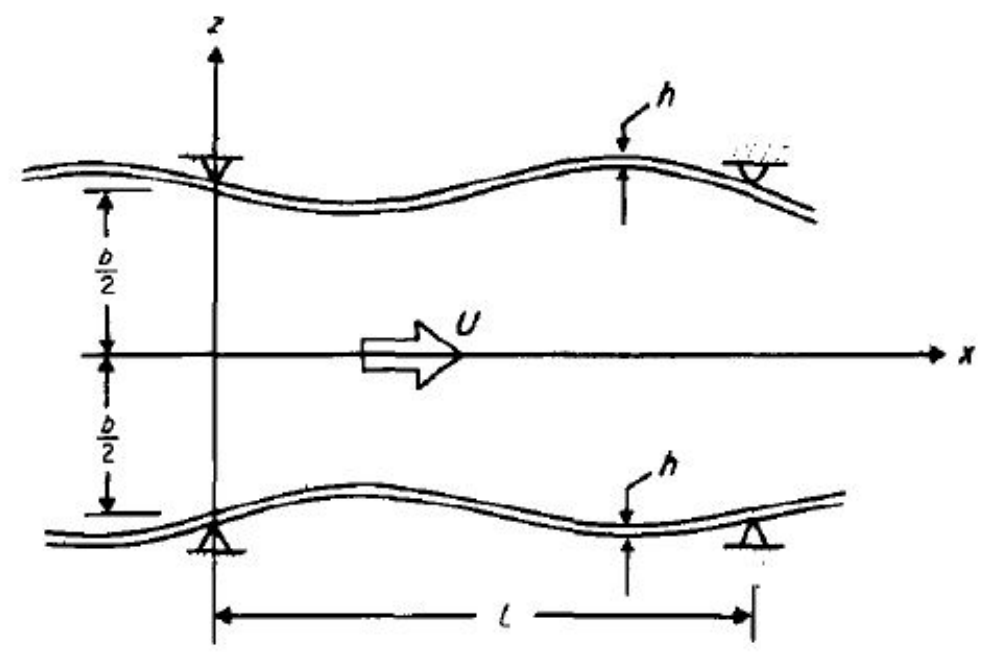

Fonte: Weaver e Paidoussis (1977).

Junto com os modelos teóricos, foram realizados experimentos para correlação. O modelo que considera largura e comprimento finitos (primeiro modelo) conseguiu representar melhor o comportamento observado nos experimentos. Foram encontrados dois possíveis modos distintos de flutter. O primeiro, nomeado de flapping mode flutter, resultou em uma divergência simétrica de dois lados adjacentes dos tubos e o segundo, definido como sendo o classic mode flutter, em cascas. O flapping mode flutter foi considerado crítico para tubos de paredes finas e qualquer estreitamento possível na tubulação diminui os valores de velocidade crítica de escoamento.

Em Tang e Dowell (1988), é estudado o modelo do tubo flexível, posicionado na vertical, engastado na extremidade superior e livre na inferior e sujeito ao escoamento axial interno. Duas placas magnéticas foram posicionadas na lateral direita e esquerda da extremidade livre do tubo (ver, Figura 6). O comportamento caótico em virtude da instabilidade por flutter ou resposta forçada é estudado em modelos teóricos e experimentais.

Ainda nesse último trabalho, é mostrado que a restrição imposta na extremidade livre do tubo pelas forças magnéticas podem desencadear instabilidade por flambagem. No problema do tubo engastado-livre não ocorre o efeito de flambagem, apenas instabilidade por flutter. Também foram discutidos os efeitos do amortecimento estrutural e do números de modos no comportamento caótico induzido pela instabilidade por flutter. 
Figura 6 - Modelo do tubo engastado-livre proposto.

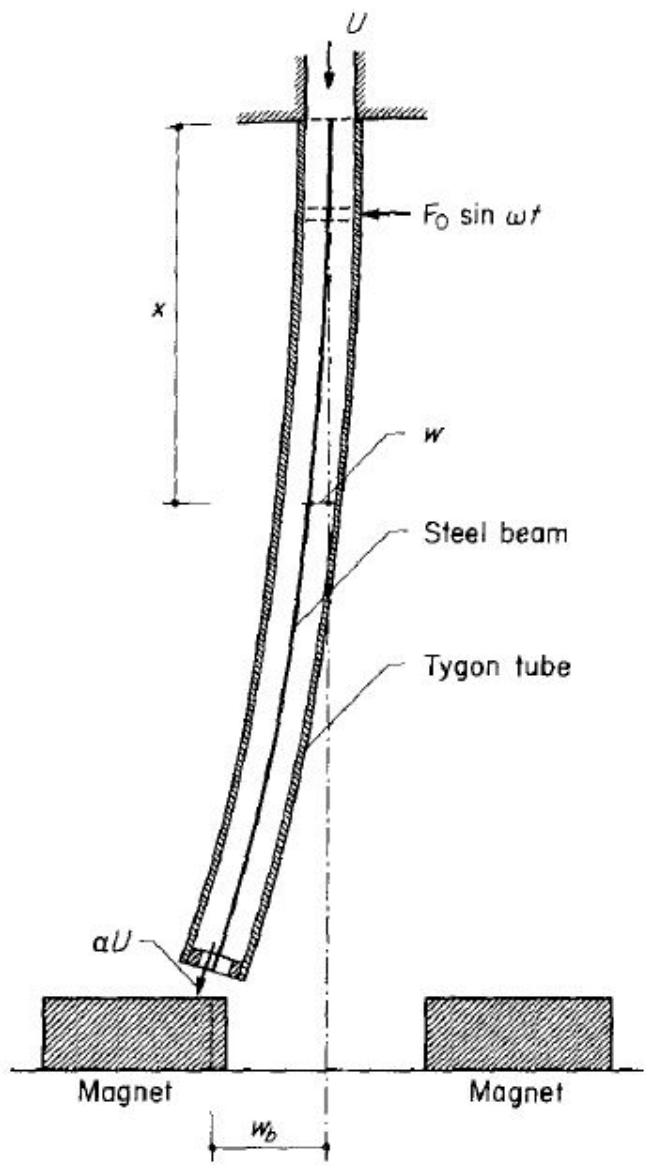

Fonte: Tang e Dowell (1988).

O estudo da estabilidade considerando as equações não lineares para o problema do pêndulo duplo com escoamento interno pulsante foi realizado por Jensen (1999). Nesse trabalho, a frequência de pulsação considerada é muito maior do que as frequências naturais do sistema. Para as situações com baixa amplitude de oscilação, foi utilizado o método das múltiplas escalas para solução do modelo. Os casos com grande amplitude foram estudados por meio de métodos numéricos de solução. É mostrado que o escoamento pulsante com frequência muito maior que a frequência natural do sistema tem a capacidade de diminuir e até mesmo suprimir as amplitudes de vibração do sistema, como mostra a Figura 7.

Na Figura 7 foram apresentadas as respostas dinâmicas levando em consideração a existência do escoamento pulsante. Na resposta identificada pela letra (a) o autor adotou o escoamento constante no tempo e chamou atenção para a amplitude de oscilação do sistema. Nas séries identificadas pelas letras (b) e (c), considerou-se o escoamento pulsante com velocidade média igual àquela que produziu a figura (a) e foram aplicadas duas condições de perturbação inicial distintas. Na série identificada por (b), o autor aplica uma pequena perturbação e observa que as amplitudes de oscilação são amortecidas. já na série identificada em (c) a perturbação inicial é cinco vezes maior do que a considerada 
Figura 7 - Comportamento do sistema com e sem escoamento pulsante.
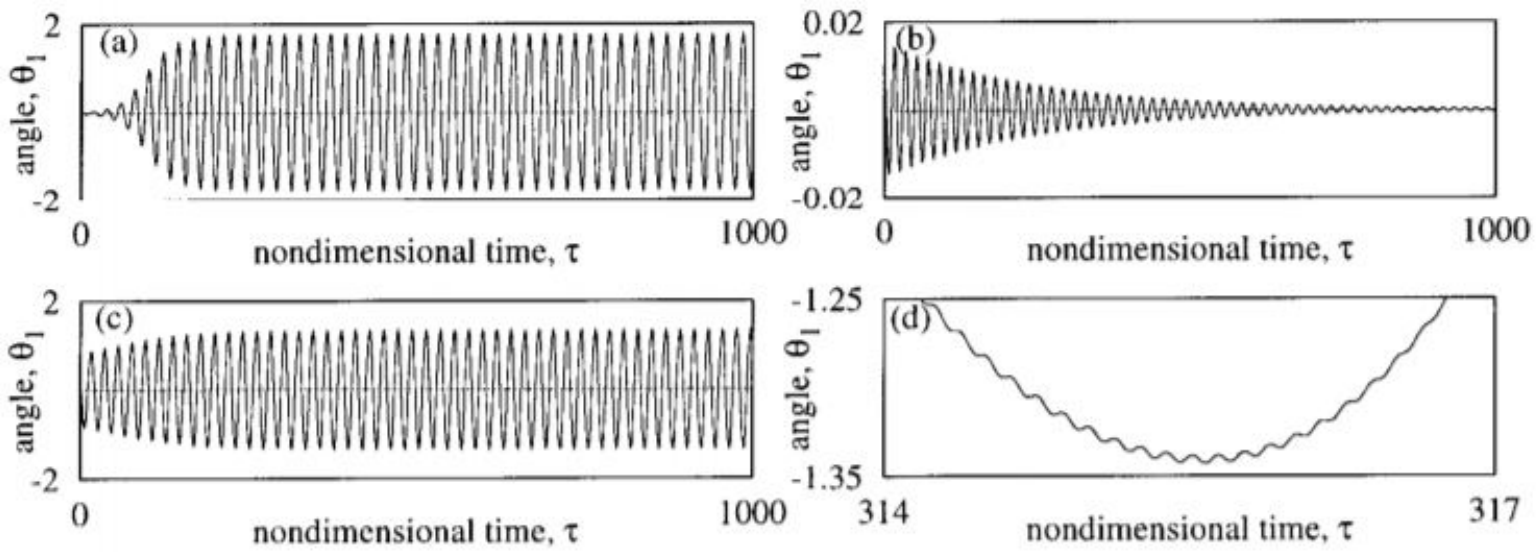

Fonte: Jensen (1999).

em (b) e as amplitudes diminuem comparadas com o caso identificado por (a) mas não são amortecidas. Por sua vez, no caso indicado por (d), o autor diminui a escala do adimensional de tempo para mostrar a modulação na resposta causada pelo escoamento pulsante.

No trabalho desenvolvido por Lee e Chung (2002), é proposto um novo modelo para o problema do tubo reto, com escoamento interno e com as duas extremidades fixas. Foram consideradas as teorias de barras de Euler-Bernoulli, além do princípio estendido de Hamilton para obtenção das equações não lineares de movimento. Essas equações foram discretizadas utilizando o método de Galerkin e posteriormente linearizadas para a avaliação dos resultados em torno da posição de equilíbrio. A validação dos resultados foi feita comparando as respostas obtidas com as equações propostas por Païdoussis.

Em Sadeghi e Païdoussis (2009), foi estudado o problema do escoamento interno em um tubo extensível com as duas extremidades fixas. Foi realizada a discretização do sistema de equações diferenciais via método de Galerkin. O objetivo desse trabalho foi verificar o comportamento do sistema em torno da posição de equilíbrio para velocidades de escoamento superiores a que causariam perda de estabilidade por divergência estática após ponto de bifurcação. Na Figura 8 são mostrados os mapas de bifurcação encontrados considerando as condições de apoio fixo nas duas extremidades em (a), engastamento nas duas extremidades em (b) e engastado-fixo em (c). É possível notar que após o ponto de bifurcação o sistema passa a ter 3 possibilidades de respostas, 2 sendo estáveis e 1 instável.

Kheiri et al. (2014) estudam a dinâmica e a estabilidade de um tubo contínuo com escoamento interno apoiado em suportes elásticos (ver Figura 9). Nesse trabalho é resolvida uma série de modelos intermediários com diferentes condições de contorno que possibilitaram comparar as respostas com as obtidas para o modelo apresentado na Figura 9 . 
Figura 8 - Mapa de bifurcação.
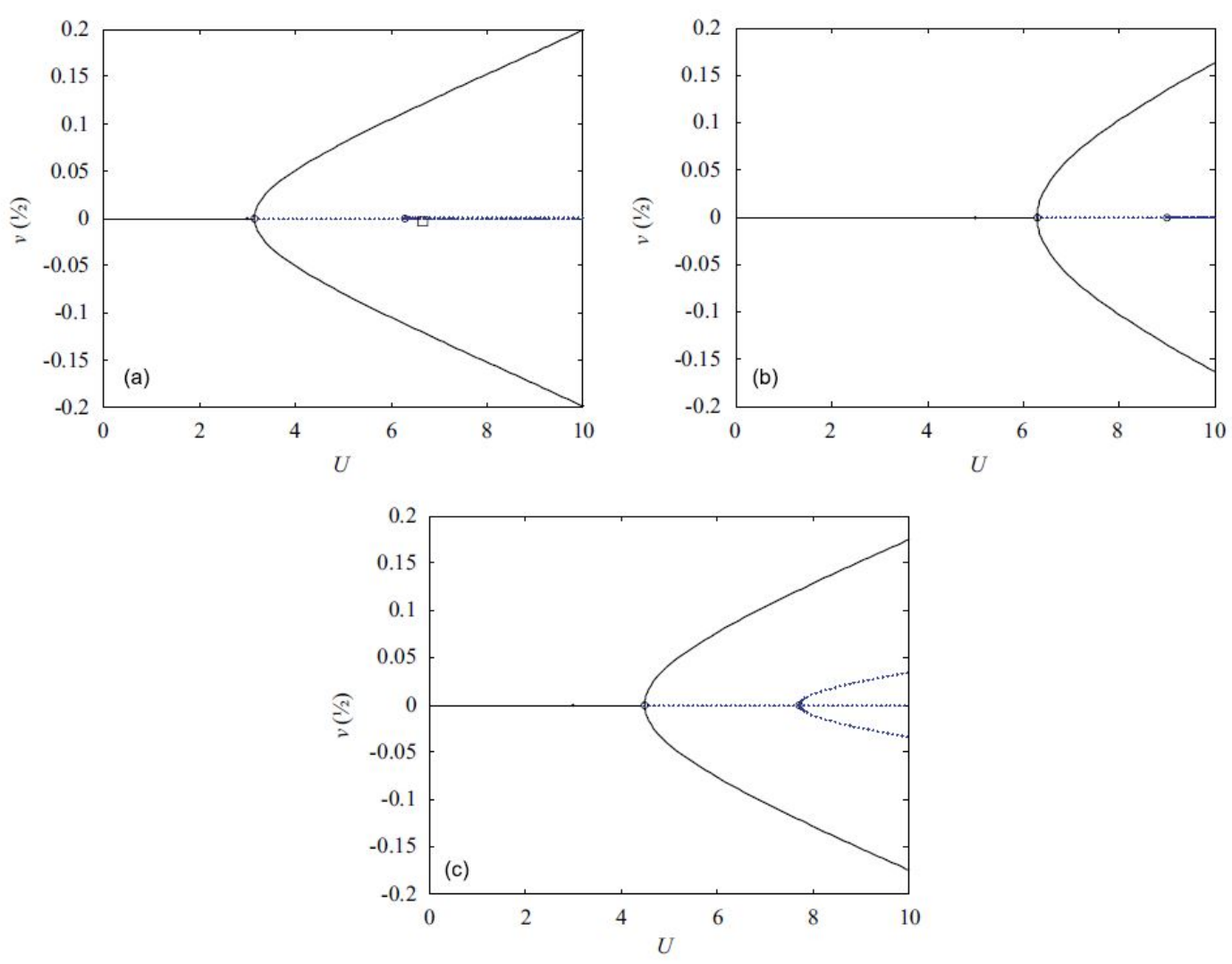

Fonte: Sadeghi e Païdoussis (2009).

Quando o modelo proposto na Figura 9 foi analisado, foi mostrado que adotar molas rotacionais e translacionais com grande rigidez em ambos os apoios não altera o valor da velocidade crítica do sistema. Verificou-se que existe valor de rigidez limite, no qual, a partir dele a velocidade crítica de escoamento não muda. É mostrado por Kheiri et al. (2014) que, para valores baixos de rigidez, o sistema se comporta como um tubo com as extremidades livres, onde o valor de velocidade crítica torna-se cada vez menor até finalmente se aproximar de zero.

Em Orsino e Pesce (2018) é apresentada uma nova forma de resolver o problema do tubo com escoamento axial utilizando a metodologia desenvolvida em Orsino (2016). Essa metodologia consegue explorar as simetrias e modularidades de um dado sistema dinâmico, fornecendo um algoritmo no qual as restrições cinemáticas podem ser adotadas a posteriori respeitando os operadores de projeção adequados.

Além de todos os artigos aqui mencionados, o livro ${ }^{1}$ Païdoussis (1998) é fundamental para o estudo de vibrações induzidas pelo escoamento axial. Nele são realizadas análises em modelos linearizados contínuos e discretizados, além de modelos não lineares. As

1 O livro Païdoussis (1998) organiza anos de estudos no tema realizados pelo grupo liderado pelo Prof. Païdoussis. 
Figura 9 - Tubo contínuo com escoamento interno apoiado em suportes elásticos.

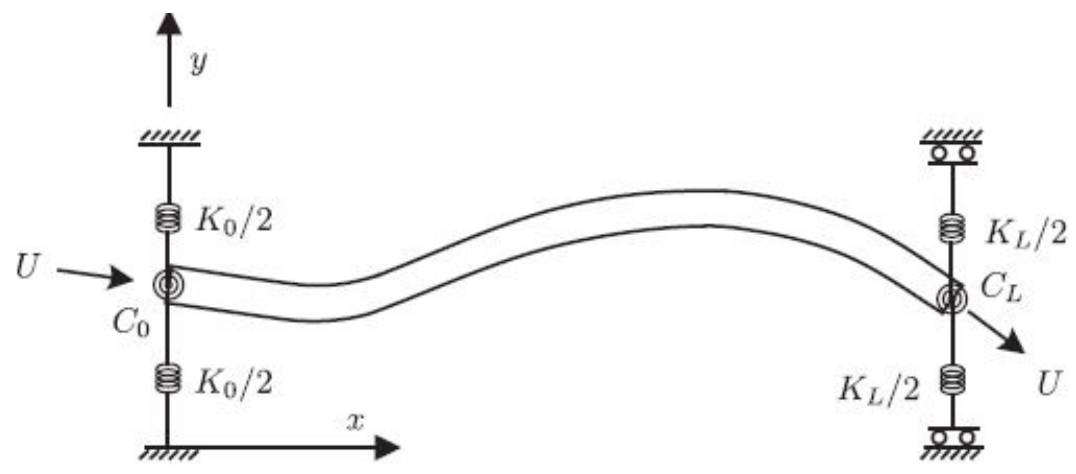

Fonte: Kheiri et al. (2014).

equações matemáticas encontradas na pesquisa aqui descrita foram comparadas com os resultados de problemas similares presentes no livro e serão discutidos no Capítulo 3. Em seu livro, Païdoussis encontrou a equação não linear para o mesmo problema estudado por Benjamin. As equações não lineares (ver Equações 2.3 e 2.4) servirão como referência e serão comparadas com as expressões obtidas na Seção 3.3, que consideram também a excitação de suporte em adição ao efeito do escoamento interno.

Nesta seção foram apresentados alguns dos trabalhos desenvolvidos ao longo das últimas décadas. Por se tratar de uma quantidade considerável de artigos desenvolvidos no assunto, fica a sugestão da leitura dos artigos Ibrahim (2010) e Ibrahim (2011), ambos responsáveis por apresentar uma abrangente revisão bibliográfica no tema.

$$
\begin{aligned}
& (M+m) g\left(\frac{1}{2} L_{1}^{2}+L_{1} L_{2}\right) \sin \theta_{1}+ \\
& +(M+m)\left[-\frac{1}{2} L_{1} L_{2}^{2} \dot{\theta}_{2}^{2} \sin \left(\theta_{2}-\theta_{1}\right)+\ddot{\theta}_{1}\left(\frac{1}{3} L_{1}^{3}+L_{1}^{2} L_{2}\right)+\frac{1}{2} L_{1} L_{2}^{2} \ddot{\theta}_{2} \cos \left(\theta_{2}-\theta_{1}\right)\right]+ \\
& +2 M U\left[\frac{1}{2} L_{1}^{2} \dot{\theta}_{1}+L_{1} L_{2} \dot{\theta}_{2} \cos \left(\theta_{2}-\theta_{1}\right)\right]+k_{1} \theta_{1}-k_{2}\left(\theta_{2}-\theta_{1}\right)+ \\
& +M L_{1} L_{2} \dot{U} \sin \left(\theta_{2}-\theta_{1}\right)+M U^{2} L_{1} \sin \left(\theta_{2}-\theta_{1}\right)=0 \\
& \frac{1}{2}(M+m) g L_{2} \sin \theta_{2}+\frac{1}{3}(M+m) L_{2}^{3} \ddot{\theta}_{2}+M U L_{2}^{2} \dot{\theta}_{2}+\frac{1}{2}(M+m) L_{1} L_{2}^{2} \ddot{\theta}_{1} \cos \left(\theta_{2}-\theta_{1}\right)+ \\
& +k_{2}\left(\theta_{2}-\theta_{1}\right)+\frac{1}{2}(M+m) L_{1} L_{2}^{2} \dot{\theta}_{1}^{2} \sin \left(\theta_{2}-\theta_{1}\right)=0
\end{aligned}
$$

\subsection{Excitação paramétrica}

O problema de excitação paramétrica ocorre quando um ou mais parâmetros ${ }^{2}$ da equação de movimento dependem explicitamente do tempo. A equação de Hill representa uma equação diferencial ordinária de segunda ordem que possui o termo de rigidez variando

\footnotetext{
2 Por exemplo, massa, rigidez ou amortecimento.
} 
com o tempo. Quando essa variação temporal é expressa por uma função harmônica e monocromática, ela então passa a ser chamada de equação de Mathieu (ver Equação 2.5).

$$
\ddot{x}+(\delta+2 \epsilon \cos 2 \tau) x=0
$$

No caso da equação de Mathieu, a excitação paramétrica pode levar a situações de instabilidade da solução trivial. Para determinadas combinações de amplitude e frequência do termo de rigidez, a solução trivial pode ser estável ou instável, a última configurando a instabilidade paramétrica. A instabilidade paramétrica é relevante quando se estuda dinâmica de risers, pois essas são estruturas extremamente esbeltas sujeitas a movimentos de topo decorrentes das respostas de primeira e segunda ordem das unidades flutuantes. Esses movimentos podem induzir forças normais dependentes do tempo.

O movimento imposto no topo do sistema pode afetar o termo de rigidez de diferentes maneiras de acordo com as condições de contorno do sistema. Para tubos com uma das extremidades livre, o termo associado à excitação paramétrica aparece como uma aceleração que corrige a aceleração da gravidade. Essa correção pode aumentar ou diminuir a rigidez do conjunto. Em casos onde o tubo possui as extremidades fixas, o termo associado à excitação paramétrica aparece como uma força normal com intensidade variável. Quanto maior o esforço de tração, maior a rigidez do sistema.

Uma das ferramentas mais utilizadas para mapear as regiões de estabilidade e instabilidade associadas à equação de Mathieu é o diagrama de Strutt, ilustrado na Figura 10. Nesse diagrama, o plano de parâmetros de controle da excitação paramétrica (a saber, sua amplitude e frequência) é divido em regiões de soluções limitadas ou ilimitadas. Os livros Nayfeh e Mook (1979) e Meirovitch (2003) e a tese Franzini (2019) trazem a formas de obtenção do diagrama de Strutt.

O diagrama de Strutt foi obtido a partir da equação de Mathieu (ver, Equação 2.5). Ele mapeia as regiões de solução limitada e ilimitada de acordo com o par de frequência e amplitude $(\delta ; \epsilon)$ adotado. Na Figura 10, a região em branco representa a área onde a solução é limitada, enquanto a região preenchida por hachura representa os pares de coordenadas que possuem solução ilimitada.

As fronteiras são curvas no plano de parâmetros $(\delta ; \epsilon)$ representadas por linhas sólidas e determinam os valores limites de frequência nos quais o sistema é instável independente da amplitude. Nesse diagrama pode-se identificar o problema de instabilidade paramétrica principal, a qual ocorre quando a frequência do problema de excitação paramétrica é o dobro da frequência natural do sistema, ocorrendo quando $\delta=1$.

O problema de instabilidade paramétrica vem sendo estudado exaustivamente no segmento de engenharia oceânica. Um dos primeiros a estudar excitação paramétrica em TLPs (Tension Leg Plataform) foi Rainey (1977), que demonstrou que as zonas de 
Figura 10 - Diagrama de Strutt.

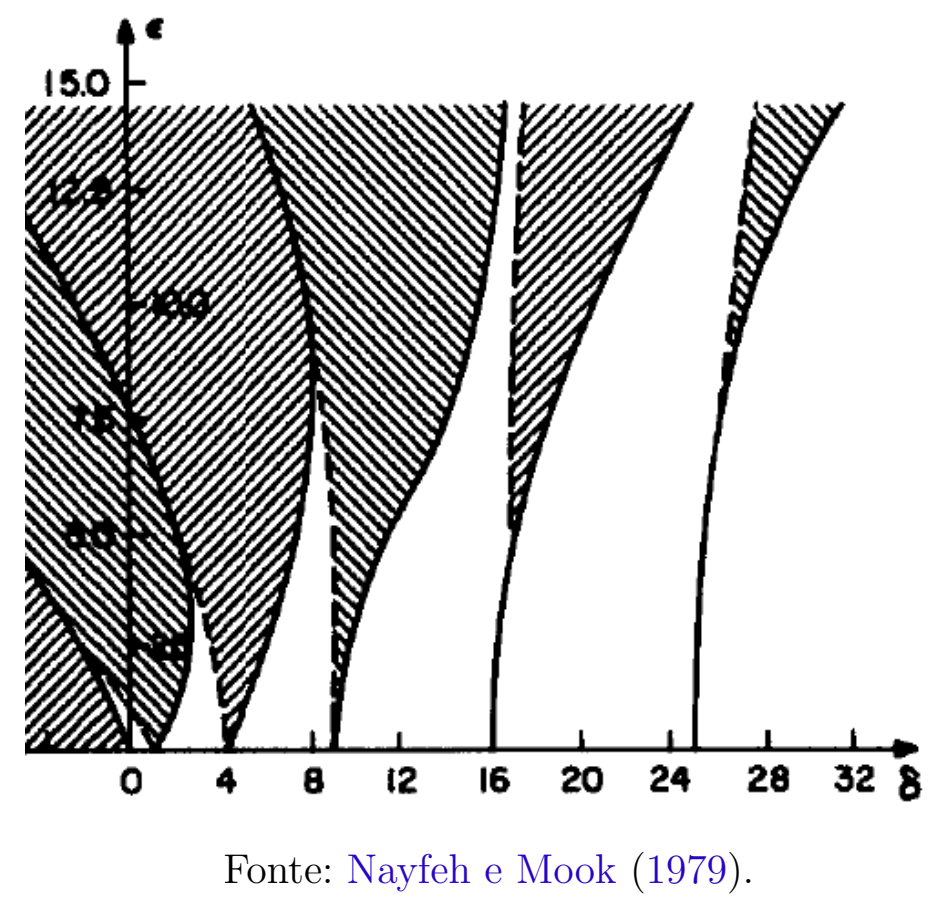

instabilidade do diagrama de Strutt para o problema de um grau de liberdade analisado aderem com resultados experimentais. Em Patel e Park (1991), foi analisado o problema dos cabos protendidos de amarração para uma plataforma TLP em águas profundas considerando diferentes condições de pré-tensão nos cabos, desde uma pré-tensão constante no comprimento até a situação sem pré-tração. Foram verificados que, para condições de instabilidade, as amplitudes de respostas são limitadas pelas forças de amortecimento hidrodinâmicas não lineares, mas cada amplitude máxima varia de acordo com os pares de parâmetros de Mathieu escolhidos. No caso que foram considerados os cabos afrouxados, a amplitude máxima obtida nas regiões de estabilidade é igual a zero.

Já em Simos e Pesce (1997) foi considerada a variação da tração ao longo do comprimento do cabo na formulação das equações de movimento. Comparando os resultados obtidos em Simos e Pesce (1997) com os discutidos em Patel e Park (1991), foi verificado que considerando a varição de tração média ao longo do comprimento, seria possível diminuir os valores de pré-tração aplicados ao cabo. O modelo desenvolvido por Simos e Pesce (1997) possui maior relevância em análises de TLP's instaladas em água profundas.

Em Chatjigeorgiou e Mavrakos (2002) é estudada a resposta dinâmica não linear dos deslocamentos transversais de risers ou cabos de amarração pré tracionados posicionados na vertical submetidos a excitação paramétrica junto ao topo da estrutura. A abordagem analítica revelou que a resposta dinâmica dos deslocamentos laterais é governada pelo efeito de acoplamento modal na direção transversal. O resultado mostra que o acoplamento dos modos alterna as regiões estáveis e instáveis para o caso sem amortecimento. Também 
é mostrado que os movimentos laterais são dominados pelo primeiro modo de vibração, os quais respondem de forma sub-harmônica comparado com a frequência de excitação paramétrica.

No trabalho desenvolvido por Koo, Kim e Randall (2004) é estudada a instabilidade de Mathieu em plataformas do tipo spar. É mostrado que as amplitudes de movimento aumentam quando ocorre a variação harmônica nos coeficientes de restauração do grau de liberdade pitch causados por movimentos de grande amplitude na direção de heave. Isso ocorre quando o período de movimento de heave é metade do período natural de oscilação de pitch $^{3}$.

Em Chandrasekaran, Chandak e Anupam (2006) é realizada a análise dinâmica dos cabos de fixação de plataformas do tipo TLP considerando a variação linear na intensidade da pré-tração ao longo do comprimento do cabo. A estabilidade de Mathieu foi verificada para plataformas de diferentes formatos e profundidades do leito oceânico. Os modos de vibrar do cabo de fixação foram obtidos através de funções de Bessel. Os resultados obtidos por Chandrasekaran, Chandak e Anupam (2006) mostram que TLPs instaladas em águas profundas possuem menores amplitudes de oscilação quanto maior é o valor de pré-tração adotada.

Em Chatjigeorgiou (2007), é estudado o problema de altas tensões no material ocasionadas pelos esforços de flexão oriundos de grandes deslocamentos laterais por conta dos efeitos dinâmicos aos quais os cabos e risers estão submetidos. O modelo proposto é de uma barra esbelta posicionada na vertical com as duas extremidades engastadas. Por ter sido considerada a situação das duas extremidades engastadas, Chatjigeorgiou (2007) utilizou da metodologia de solução por técnica de camada limite apresentada em Nayfeh (1985) para obter uma aproximação assintótica para os modos de vibrar do problema. Cálculos comparativos mostraram que o aumento da tração média aplicada no topo da estrutura altera o ponto de deslocamento lateral máximo da extremidade inferior do cabo para o centro da estrutura. Além disso, baixos valores de rigidez flexional ocasionam maiores tensões próximas à extremidade inferior da estrutura.

Em Franzini et al. (2015), foi realizada a análise experimental de um cilindro flexível, posicionado na vertical e submerso. Foi estudada a resposta dinâmica quando esse sistema estava exposto a uma excitação harmônica no topo. Os movimentos verticais aplicados no topo possuíam amplitudes de aproximadamente $1 \%$ do comprimento indeformado do cilindro. Foram monitoradas as respostas para quatro frequências distintas no movimento imposto $f_{t}: f_{N}=1: 3,1: 1,2: 1$ e $3: 1$, sendo $f_{t}$ a frequência do movimento imposto e $f_{N}$ a primeira frequência natural do cilindro. Para monitorar o experimento, 43 pontos ao longo do cilindro tinham suas respostas coletadas por um sofisticado sistema de rastreamento

3 Um dos seis graus de liberdade de uma embarcação. O heave é um movimento de translação na direção vertical. Já o pitch é um movimento angular em torno de um eixo que define o plano horizontal. 
óptico.

Figura 11 - Mapas de amplitude dos deslocamentos laterais

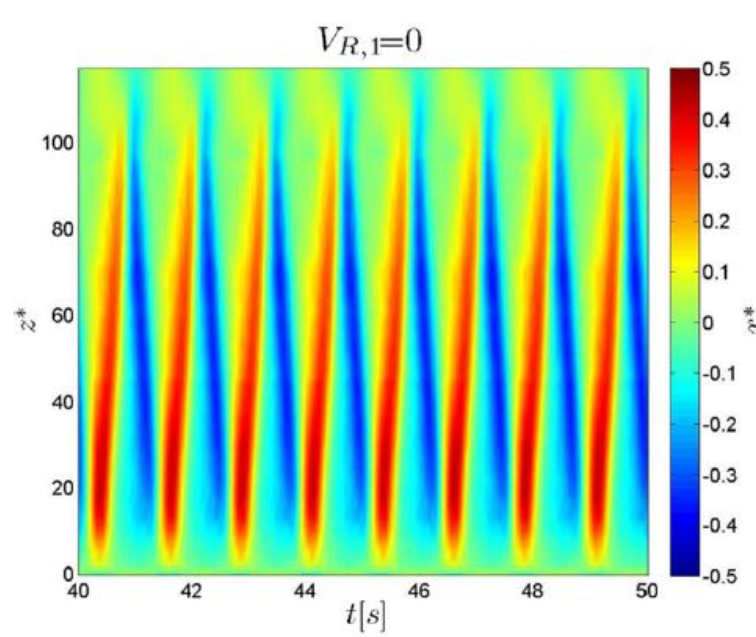

(a) $f_{t}: f_{N}=1: 1$.

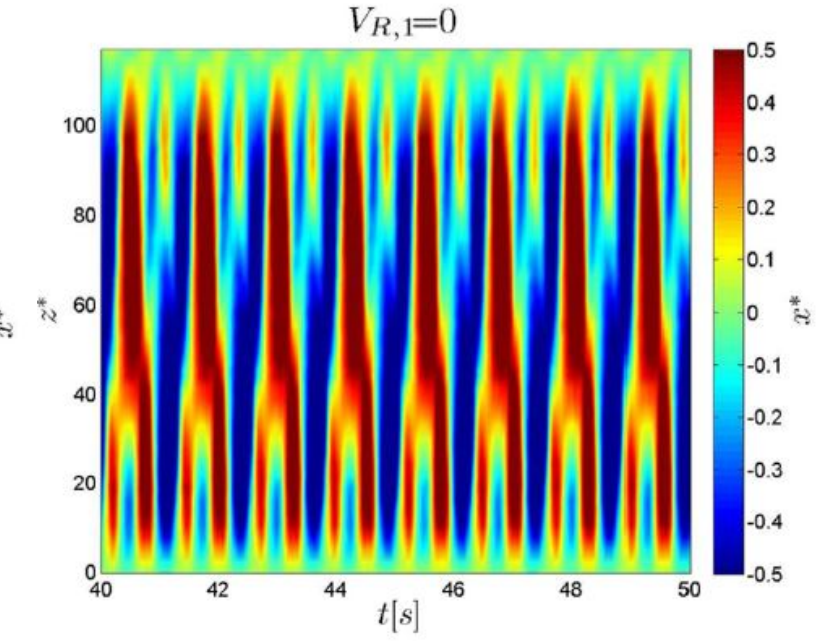

(b) $f_{t}: f_{N}=2: 1$.

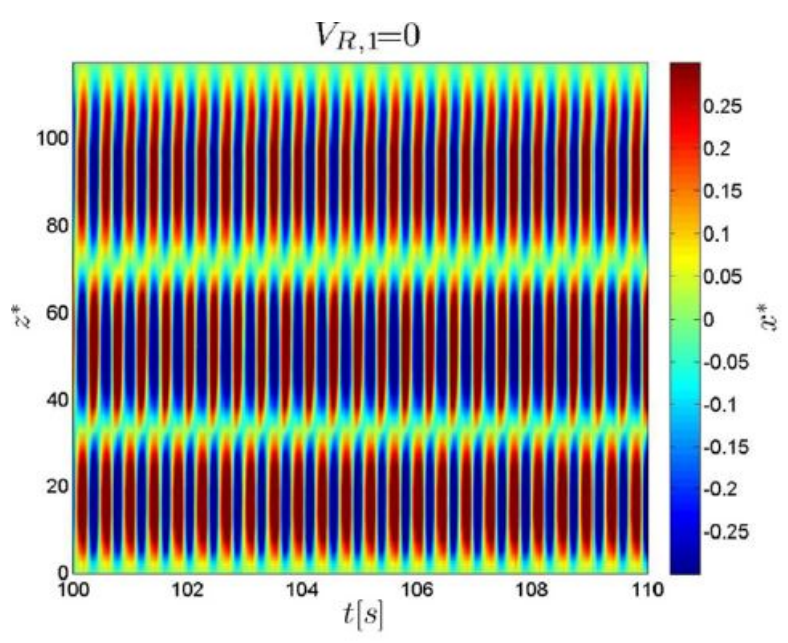

(c) $f_{t}: f_{N}=3: 1$.

Fonte: Franzini et al. (2015).

Para a relação de frequência $f_{t}: f_{N}=1: 1$ o mapa de amplitudes apresentou o padrão de pulso de onda através do cabo com predominância nos dois primeiros modos de vibrar (ver Figura 11a). Na relação $f_{t}: f_{N}=2: 1$ o mapa de amplitude indicou predominância da resposta no primeiro e segundo modos, mas o diagrama revelou um padrão no formato "de garfo" apresentado na Figura 11b. Na relação $f_{t}: f_{N}=3: 1$ é predominante o padrão de onda estacionária com o formato do terceiro modo, como mostra a Figura 11c.

O trabalho Franzini e Mazzilli (2016) propõe um modelo de ordem reduzida não linear empregado o método de Galerkin com três modos na expansão para a análise de uma barra esbelta submersa sujeita a excitação paramétrica. Foram apresentados gráficos que mostram a amplitude dos deslocamentos modais máximos para cada combinação de 
frequência e amplitude do movimento imposto ao topo. Destacam-se, ainda, as respostas nas regiões críticas onde a frequência do movimento imposto é o dobro da frequência natural do modo analisado.

Já em Vernizzi, Franzini e Lenci (2019) foi estudada a influência da função de forma utilizada no método de Galerkin em um modelo composto por uma barra flexível posicionada na vertical e submersa em fluido. Três modelos de ordem reduzida foram obtidos, no primeiro os modos de vibrar da barra foram escritos a partir das funções "Quase-Bessel" (ROM ${ }^{4}$ i), no segundo a obtenção dos modos foi baseada em uma única função trigonométrica (ROM ii) e no terceiro foram utilizadas três funções trigonométricas como um conjunto de funções de forma (ROM iii).

Figura 12 - Comparação dos espaços de fase da solução quase estática para diferentes amplitudes de excitação paramétrica com frequência adimensional $n=2,0$.

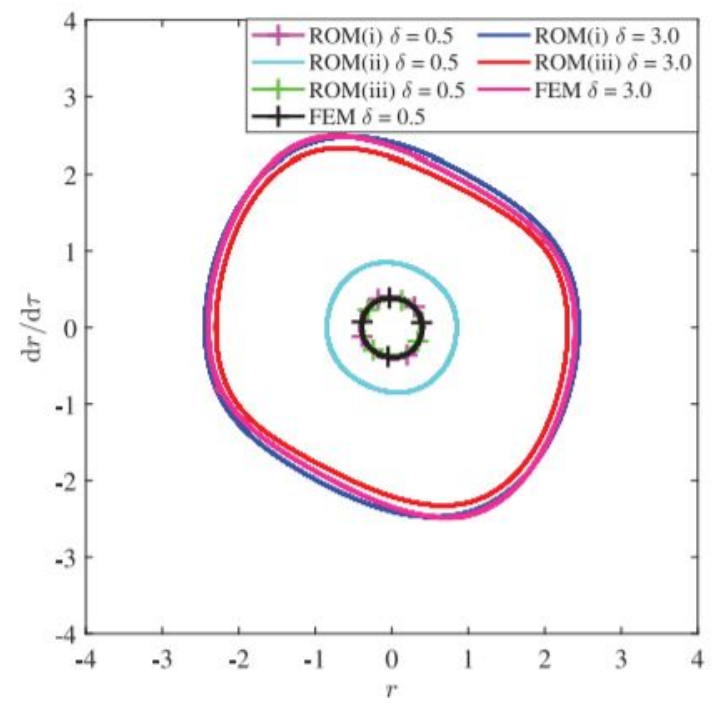

Fonte: Vernizzi, Franzini e Lenci (2019).

Os resultados dos três modelos de ordem reduzida (ROM i, ROM ii e ROM iii) foram comparados com resultados obtidos através da simulação do mesmo modelo via método dos elementos finitos $\left(\mathrm{FEM}^{5}\right)$ e esses resultados podem ser observados na Figura 12. É mostrado que o modelo que utilizou as funções "Quase-Bessel" (ROM i) apresentou bons resultados comparado com o modelo em elementos finitos (FEM), isso garante a aceitação da hipótese de negligenciar a dinâmica axial do sistema.

Já os modelos baseados nas funções trigonométricas mostraram que para um resultado aceitável é necessário utilizar mais de uma função de forma (ROM ii e ROM iii). Na Figura 12 é mostrado que o modelo baseado em uma função de forma trigonométrica apresentou resultados diferentes quando comparado com aqueles obtidos via método dos elementos finitos (FEM).

4 Do inglês, reduced-order model

5 Do inglês, finite element method 


\subsection{Efeitos combinados de escoamento interno e excitação para- métrica}

Com o aumento contínuo do consumo dos combustíveis fósseis foi necessário começar a exploração de gás e petróleo em locais com maior dificuldade de extração. Dessa forma, ocorreu também a necessidade de avanços nas pesquisas científicas para garantir a segurança e eficiência nas realizações desses processos. Pesquisas no assunto vêm sendo realizadas desde as décadas de 40 e 50. Os fenômenos físicos que solicitam as estruturas foram estudados de forma separada durante anos. Com o aumento das profundidades de extração e consequentemente dos comprimentos dos risers, pesquisas que combinam mais de um destes efeitos passaram a ser realizadas.

No problema tecnológico existe concomitância entre os efeitos de vibração induzida pelo escoamento interno, a vibração induzida por vórtices (VIV) e o problema de instabilidade paramétrica. Tanto o problema de escoamento interno e instabilidade paramétrica já foram discutidos de maneira separada nas Seções 2.1 e 2.2.

A combinação entre os efeitos de escoamento interno e excitação paramétrica pode ocorrer de algumas formas. O trabalho apresentado por esta dissertação trata a excitação paramétrica como sendo resultado de um movimento harmônico imposto no suporte. Outros trabalhos na literatura têm como fonte do problema de excitação paramétrica a variação dos esforços de tração no tubo e também a variação do fluxo de escoamento interno. Os trabalhos apresentados a seguir podem possuir qualquer um dos três motivos citados neste paragrafo.

Na investigação desenvolvida por Bohn e Herrmann (1974), foi estudado o problema plano do pêndulo duplo considerando que o escoamento não é constante ao longo do tempo, apresentando pequenas oscilações em sua velocidade. É mostrado que o sistema pode perder estabilidade por divergência (instabilidade estática) ou por flutter (instabilidade dinâmica). Foi utilizada a Teoria de Floquet para ilustrar as diferenças entre os tipos de perda de estabilidade e mostrar que essas perturbações periódicas na velocidade do escoamento interno são responsáveis por desestabilizar o sistema.

No trabalho proposto por Rousselet e Herrmann (1978), foi estudado o problema plano do pêndulo duplo conectado a um reservatório e sujeito a escoamento interno com velocidade próxima da velocidade crítica (ver Figura 13). O reservatório conectado ao conjunto de tubos pode influenciar significativamente na resposta de acordo com a sua capacidade. Pequenos reservatórios podem ter grandes amplitudes de variação de nível do conteúdo e, consequentemente, de pressão na tubulação.

O sistema proposto por Rousselet e Herrmann (1978) está sujeito à instabilidade paramétrica devido a consideração da flutuação de pressão no reservatório. A perturbação 
Figura 13 - Modelo do problema clássico de Benjamin conectado a um reservatório a jusante.

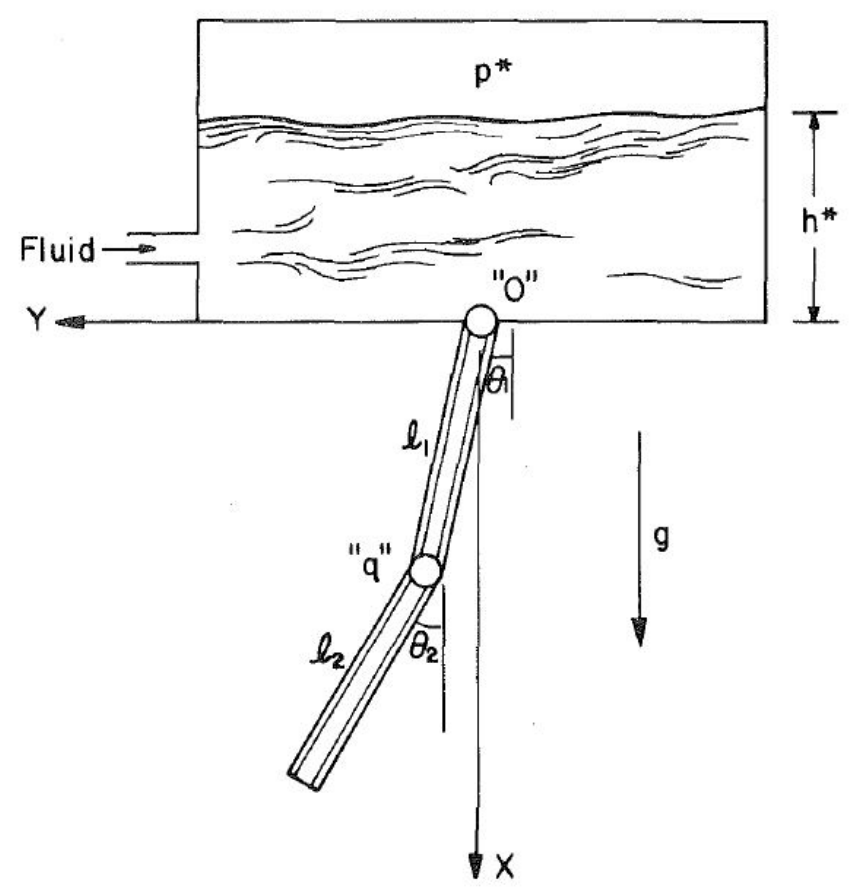

Fonte: Rousselet e Herrmann (1978).

na velocidade do escoamento é modelada como uma terceira equação que compõe as equações de movimento do sistema.

Na Figura 14, é mostrado que o sistema possui dois comportamentos distintos de acordo com o valor do adimensional de massa $\gamma^{6}$. Para valores inferiores a $\gamma=0,1$, as não linearidades atuam aumentando a amplitude da resposta em ciclo limite. Para $\gamma>0,1$ as não linearidades diminuem as amplitudes de oscilação mesmo para velocidades de escoamento maiores que a velocidade crítica.

$6 \quad$ Adimensional de massa 
Figura 14 - Resultado da variação de amplitude do ciclo limite em função do parâmetro do adimensional de massa $\gamma$.

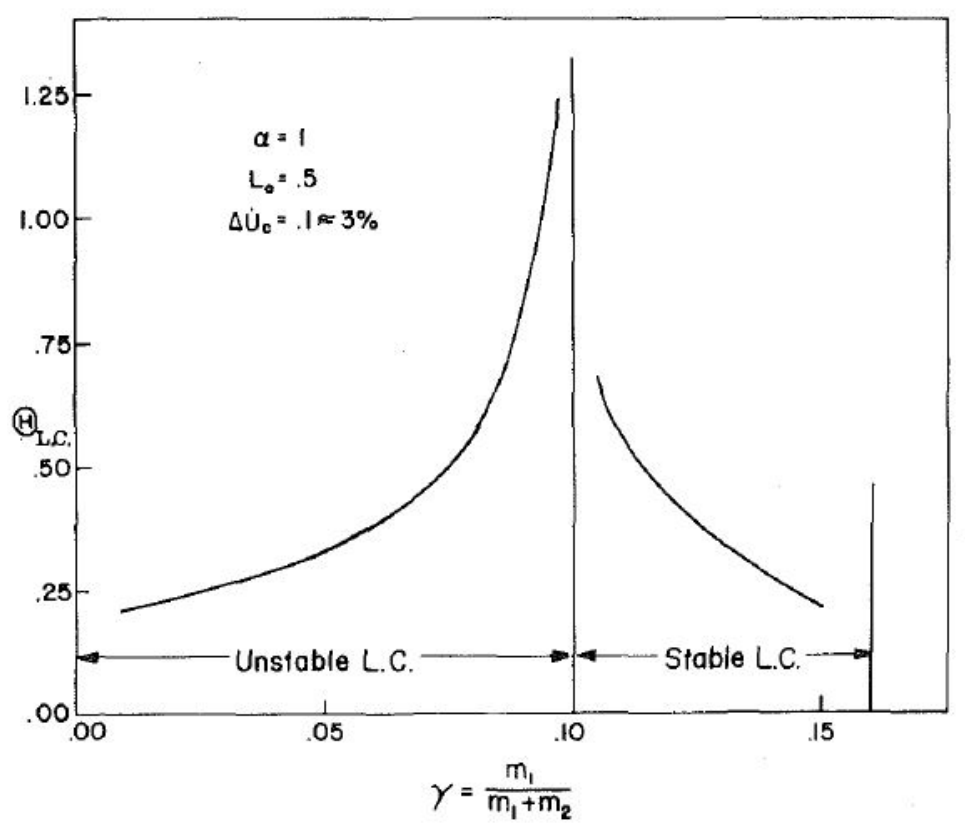

Fonte: Rousselet e Herrmann (1978).

No trabalho de Jin e Song (2005) foi estudado o problema do tubo com extremidades fixas sujeito a instabilidade paramétrica e escoamento interno pulsante no tempo. Nesse artigo, foram analisadas as mudanças em três regiões que ocorria instabilidade paramétrica quando alterados os valores de amortecimento, velocidade de escoamento e tração. Os resultados mostraram que as amplitudes de movimento crescem nas regiões de ressonância combinada (regiões onde a frequência da ação imposta é igual a soma das frequências naturais do primeiro e segundo modo de vibrar).

Panda e Kar (2008) abordam o problema não linear de um tubo articulado nas duas extremidade submetido a escoamento pulsante que ocasiona o fenômeno de excitação paramétrica e ressonância interna. A velocidade do escoamento varia com uma função harmônica e é nela que aparece o termo de excitação paramétrica do modelo matemático. As escolhas dos parâmetros foram feitas para que a frequência natural do segundo modo fosse igual a três vezes a frequência do primeiro modo. Desta forma o sistema origina a ressonância interna $3: 1$. Os resultados foram obtidos por meio da utilização do método das múltiplas escalas e apresentados por meio de séries temporais, diagramas de fase e mapas de Poincaré.

Já em Meng et al. (2018), o objeto de estudo é um cabo flexível vertical com uma extremidade fixada na plataforma e a outra no leito marinho (ver Figura 15). Nesse artigo, são considerados os efeitos de excitação de suporte e escoamento interno. É utilizada a Teoria de Floquet para verificar os efeitos dos escoamentos internos e externo no problema de instabilidade paramétrica. O trabalho Meng et al. (2018) adota, ainda, uma metodologia 
similar àquela aqui desenvolvida.

Figura 15 - Modelo do cabo flexível vertical com uma extremidade fixada na plataforma e a outra no leito marinho.

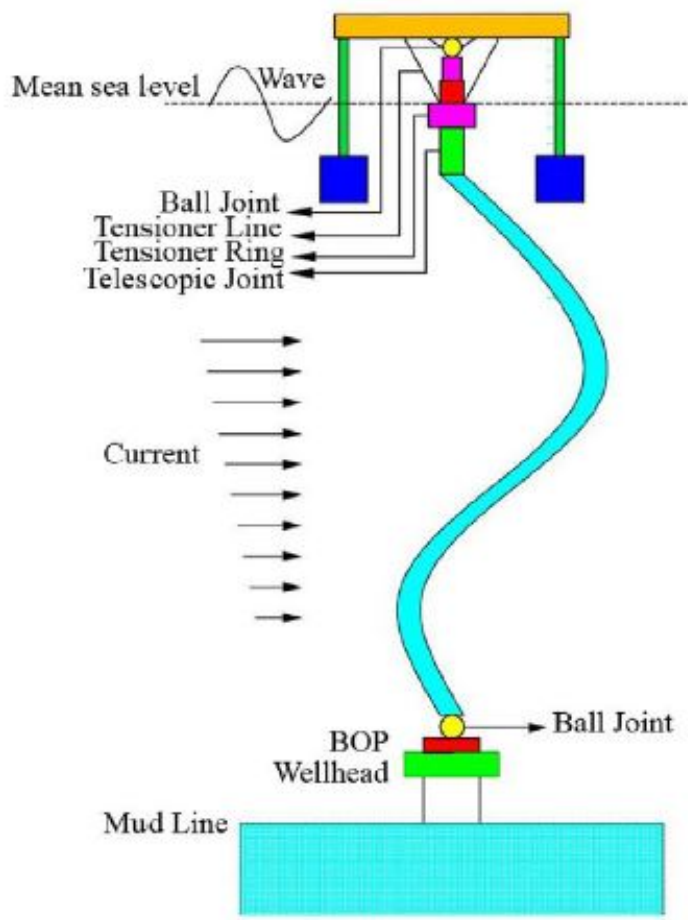

(a) Drilling riser system

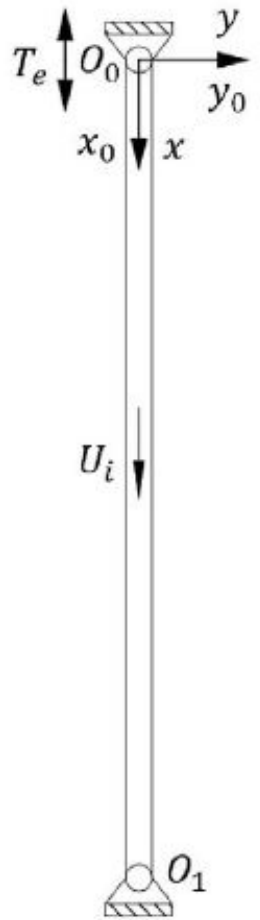

(b) Riser model

Fonte: Meng et al. (2018).

Os resultados obtidos por Meng et al. (2018) e apresentados na Figura 16 mostram análises considerando a velocidade de escoamento interno igual a 0 na Figura 16a e 10 na Figura 16b. Na Figura 16a são mostradas as zonas de instabilidade dos 6 primeiros modos excitados pelo problema de excitação paramétrica. Na Figura 16b é possível perceber que com o aumento da velocidade de escoamento interno as franjas nas zonas onde as frequências foram combinadas se aproximam do eixo das abcissas.

A principal diferença entre o trabalho aqui apresentado e o de Meng et al. (2018) é que no presente trabalho está sendo analisado o problema do tubo de Benjamin com excitação paramétrica. Por sua vez, Meng et al. (2018) estudaram o problema do tubo contínuo de grande comprimento com as duas extremidades fixas. É importante notar que a metodologia aqui adotada está no estado-da-arte, uma vez que estudos com metodologia semelhante estão sendo publicados. 
Figura 16 - Resultados a partir da análise via Teoria de Floquet.

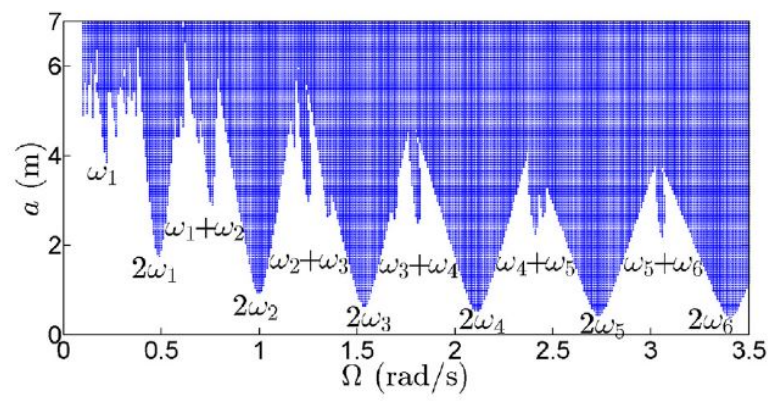

(a) $U=0$.

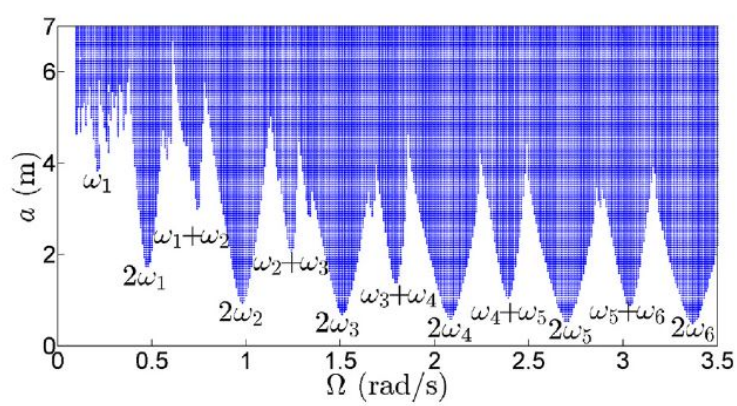

(b) $U=10$.

Fonte: Meng et al. (2018).

\subsection{Teoria de Floquet}

Para o estudo de instabilidade paramétrica, será analisado o modelo de com dois graus de liberdade. Como ferramenta foi escolhida a Teoria de Floquet. O uso dessa teoria oferece como resposta um gráfico que permite observar as regiões de instabilidade no plano de parâmetros de controle do modelo matemático.

Esse assunto é estudado de forma abrangente nos livros de Nayfeh e Mook (1979) e de Nayfeh e Balachandran (1995). As notas de aula do Prof. Richard H. Rand (ver Rand (2012)) também podem ser consultadas, pois abordam o tema de forma didática e de fácil compreensão. A seguir, será apresentada uma introdução ao tema. Na sequência, algumas particularidades do método serão discutidas durante a análise da equação de Mathieu (Seção 2.4.1).

Segue, agora, uma breve explanação acerca da teoria de Floquet, que é considerada a teoria geral para sistemas de equações diferenciais lineares não autônomas (ver Equação $2.6)$.

$$
\ddot{x}+p_{1}(t) \dot{x}+p_{2}(t) x=0
$$

onde $p_{1}(t)$ e $p_{2}(t)$ são funções periódicas de período $\bar{\tau}$. A solução da Equação 2.6 pode ser observada a seguir.

$$
x(t)=d_{1} x_{1}(t)+d_{2} x_{2}(t)
$$

sendo $x_{1}$ e $x_{2}$ são soluções linearmente independentes da Equação 2.6 e $d_{1}$ e $d_{2}$ são constantes a serem determinadas. Por se tratar de um problema não autônomo (isto é, aquele onde existe dependência explícita do tempo) é necessário verificar a solução no instante $t+\bar{\tau}$. Poderá ser visto em Rand (2012) que, mesmo $x(t+\bar{\tau}) \neq x(t), x(t+\bar{\tau})$ 
também é solução da Equação 2.6. Logo:

$$
\begin{aligned}
& \ddot{x}_{1}(t+\bar{\tau})+p_{1}(t) \dot{x}_{1}(t+\bar{\tau})+p_{2}(t) x_{1}(t+\bar{\tau})=0 \\
& \ddot{x}_{2}(t+\bar{\tau})+p_{1}(t) \dot{x}_{2}(t+\bar{\tau})+p_{2}(t) x_{2}(t+\bar{\tau})=0
\end{aligned}
$$

Adotando o mesmo raciocínio usado na Equação 2.7, pode-se escrever as respostas das Equações 2.8 e 2.9 da seguinte maneira.

$$
\begin{aligned}
& \mathbf{x}_{1}(t+\bar{\tau})=a_{11} x_{1}(t)+a_{12} x_{2}(t) \\
& \mathbf{x}_{2}(t+\bar{\tau})=a_{21} x_{1}(t)+a_{22} x_{2}(t)
\end{aligned}
$$

ou, de forma mais compacta usando notação vetorial, como:

$$
\mathbf{x}(t+\bar{\tau})=\mathbf{A} \mathbf{x}(t)
$$

$\operatorname{com} \mathbf{x}=\left\{\begin{array}{ll}x_{1} & x_{2}\end{array}\right\}^{T}$.

Com o que foi mostrado até agora é possível garantir que, a solução no instante $t+\bar{\tau}$ é combinação linear das soluções fundamentais no instante $t$. Ressalta-se, também que a matriz $\mathbf{A}$ é não singular, esta matriz não é única e varia de acordo com o conjunto de soluções fundamentais adotado.

Pode ser visto em Nayfeh e Mook (1979) que a matriz A tem dimensão $n \times \mathrm{x} n \mathrm{e}$ depende da escolha de $\mathbf{x}(t)$, podendo ser interpretada como um mapa que leva as soluções no instante $t=0$ para as soluções em $t=\bar{\tau}$. Se a escolha do conjunto fundamental no instante de tempo $t=0$ resulta em $\mathbf{X}(0)=\mathbf{I}$ é possível concluir que $\mathbf{A}=\mathbf{x}(\bar{\tau})$, denominada matriz de monodromia.

Podemos adotar um outro conjunto de soluções fundamentais $\mathbf{v}$, o qual pode ser escrito como combinação linear de x. Desta forma, a Equação 2.12 pode ser apresentada da seguinte forma.

$$
\begin{gathered}
\mathbf{x}(t)=\mathbf{P} \mathbf{v}(t) \\
\mathbf{x}(t+\bar{\tau})=\mathbf{P} \mathbf{v}(t+\bar{\tau})
\end{gathered}
$$

Substituindo a Equação 2.12 na Equação 2.14 chega-se a,

$$
\mathbf{P}_{\mathbf{v}}(t+\bar{\tau})=\mathbf{A x}(t)
$$


Realizando um pequeno trabalho algébrico e substituindo a Equação 2.13 na Equação 2.15,

$$
\mathbf{v}(t+\bar{\tau})=\mathbf{P}^{-1} \mathbf{A} \mathbf{P} \mathbf{v}(t)=\mathbf{B} \mathbf{v}(t)
$$

Segundo Nayfeh e Mook (1979) é possível provar que as matrizes A e B possuem os mesmo autovalores. A escolha de $\mathbf{P}$ pode ser feita para que $\mathbf{B}$ possua forma canônica de Jordan. Portanto a solução de Floquet pode ser vista a seguir, caso $\hat{\lambda}_{1} \neq \hat{\lambda}_{2}$.

$$
\begin{aligned}
& \mathbf{v}_{1}(t+n \bar{\tau})=\hat{\lambda}_{1}^{n} \mathbf{v}_{1}(t) \\
& \mathbf{v}_{2}(t+n \bar{\tau})=\hat{\lambda}_{2}^{n} \mathbf{v}_{2}(t)
\end{aligned}
$$

Onde $\hat{\lambda}_{i}^{n}$ representam os autovalores da matriz B. Quando $n \rightarrow \infty$, os valores de $\mathbf{v}(t)$ tenderão a zero ou ao infinito de acordo com os valores dos módulos de dos autovalores $\left(\left|\hat{\lambda}_{i}^{n}\right|\right)$, caracterizando solução limitada (estabilidade) ou solução ilimitada (instabilidade). Cada uma destas situações poderá ser visualizada a seguir.

$$
\mathbf{v}(t) \rightarrow \begin{cases}0 & \text { se todos }\left|\hat{\lambda}_{i}^{n}\right| \leq 1 \text { estabilidade } \\ \infty & \text { se ao menos um }\left|\hat{\lambda}_{i}^{n}\right|>1 \text { instabilidade }\end{cases}
$$

\subsubsection{Exemplo de aplicação à equação de Mathieu}

Como exemplo de aplicação da Teoria de Floquet, será resolvido o problema da equação de Mathieu. Essa é uma equação diferencial linear de segunda ordem, onde o parâmetro de rigidez varia harmonicamente no tempo. A equação de Mathieu representa sistemas submetidos ao problema de instabilidade paramétrica como o que será mostrado no Apêndice A.1 e é dada pela Equação 2.19.

$$
\ddot{x}+(\delta+2 \epsilon \cos 2 \tau) x=0
$$

A solução desse exercício possibilita criar um mapa que mostra se a solução trivial é estável ou instável de acordo com os valores de $\delta$ e $\epsilon$. Em outras palavras, a Teoria de Floquet é aqui empregada para a construção numérica do diagrama de Strutt.

A excitação paramétrica observada na Equação 2.19 tem período $\bar{\tau}=\pi$. Transformando essa equação diferencial de segunda ordem em um sistema de duas equações diferenciais de primeira ordem, chega-se à Equação 2.20.

$$
\left[\begin{array}{l}
\dot{x}_{1} \\
\dot{x}_{2}
\end{array}\right]=\left[\begin{array}{cc}
0 & 1 \\
-\delta-2 \epsilon \cos 2 \tau & 0
\end{array}\right]\left[\begin{array}{l}
x_{1} \\
x_{2}
\end{array}\right]
$$


A transformação da equação diferencial de segunda ordem em um sistema de equações diferenciais de primeira ordem foi realizada para possibilitar a utilização da teoria de Floquet. Como foi visto da Seção 2.4, a resolução do sistema de equações para o conjunto fundamental de soluções em $\mathbf{x}(0)=\mathbf{I}$, permite que a verificação das condições de estabilidade do sistema pode ser realizada a partir da análise da matriz de monodromia. Na Equação 2.21 são apresentadas as condições iniciais de solução da equação de Mathieu.

$$
\begin{array}{ll}
x_{I}(0)=1 & x_{I I}(0)=0 \\
\dot{x}_{I}(0)=0 & \dot{x}_{I I}(0)=1
\end{array}
$$

Isso só é possível pois a matriz de monodromia A é linearmente independente. Desta forma, a cada período do sistema não autônomo a nova matriz de soluções fundamentais é uma transformação linear da matriz $\mathbf{A}$, possuindo assim os mesmos autovalores. Integrando as equações de movimento em um intervalo de um período e solucionando este sistema diferencial de primeira ordem para cada condição inicial (ver, Equação 2.21) é possível obter os autovalores do sistema e determinar as condições de estabilidade.

$$
\mathbf{A}=\left[\begin{array}{ll}
a_{11} & a_{12} \\
a_{21} & a_{22}
\end{array}\right]=\left[\begin{array}{ll}
x_{I}(\bar{\tau}) & x_{I I}(\bar{\tau}) \\
\dot{x}_{I}(\bar{\tau}) & \dot{x}_{I I}(\bar{\tau})
\end{array}\right]
$$

A principal vantagem da Teoria de Floquet é que o sistema pode ser resolvido numericamente a partir da integração da equação de movimento entre os instantes 0 e $\bar{\tau}$. A desvantagem é a necessidade do dobro de integrações numéricas, uma vez que o sistema precisa ser resolvido para cada condição inicial possível (ver, Equação 2.21).

A Equação matricial 2.22 mostra que as respostas para a primeira condição inicial no instante $\bar{\tau}=\pi$ foram arranjadas na primeira coluna e as respostas para a segunda condição inicial foram colocadas na segunda coluna da matriz. De posse dos valores numéricos da matriz $\mathbf{A}$, é trivial calcular seus autovalores $\hat{\lambda}_{i}$. A condição de estabilidade é escrita como:

$$
x(t) \rightarrow \begin{cases}0 & \text { se todos }\left|\hat{\lambda}_{i}\right| \leq 1 ; \text { estabilidade } \\ \infty & \text { se ao menos um }\left|\hat{\lambda}_{i}\right|>1 ; \text { instabilidade }\end{cases}
$$

Com essas informações foi possível montar um código para resolver numericamente a determinação das regiões de estabilidade da equação de Mathieu. Para cada par de parâmetros de controle $(\delta ; \epsilon)$ escolhido, o sistema foi solucionado por meio da função NDSolve implementada no software Wolfram Mathematica ${ }^{\circledR}$. Essa função já identifica qual o melhor método numérico de integração de acordo com o formato das equações diferenciais. Em cada uma das vezes que a EDO foi resolvida, os resultados foram arranjados de acordo com a Equação 2.22 e os autovalores correspondentes foram calculados. 
Figura 17 - Diagrama de Strutt para equação de Mathieu.

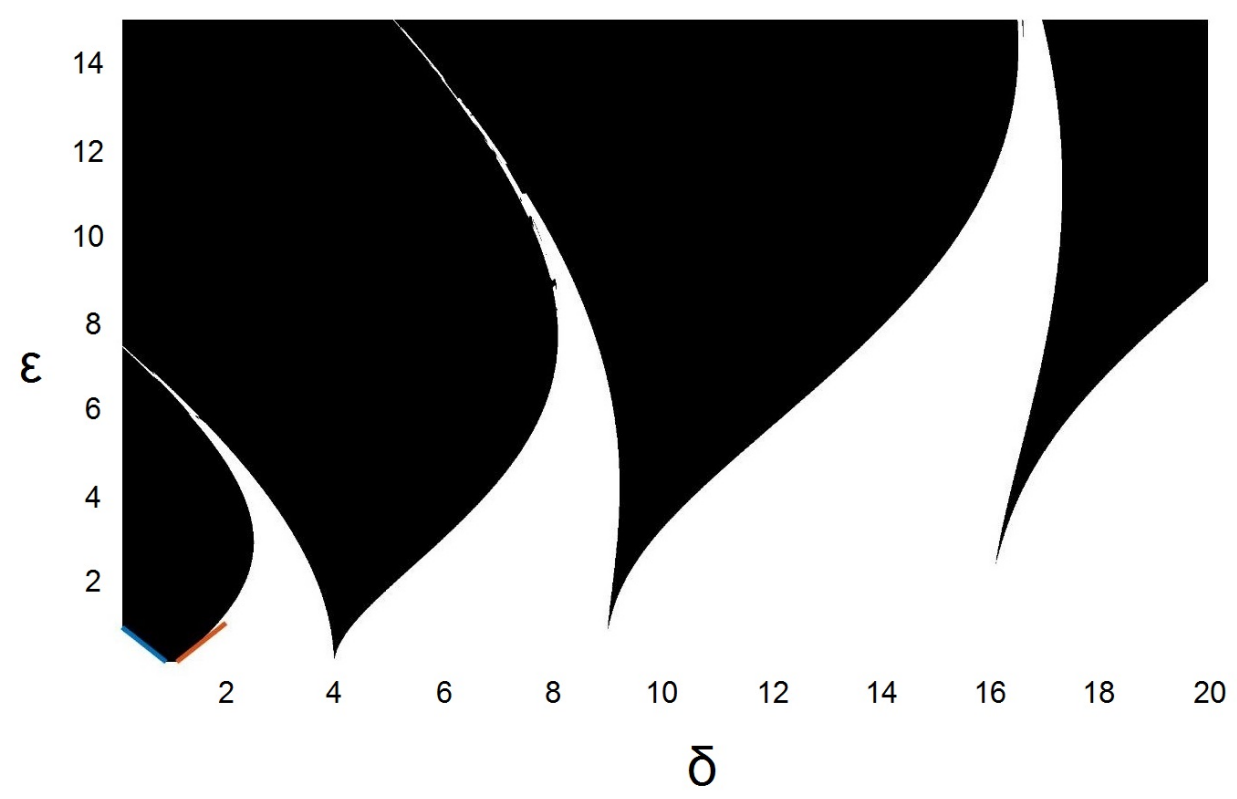

Para cada situação os autovalores foram analisados e comparados conforme foi explicado na Seção 2.4. Quando todos os módulos dos autovalores tiverem resultados menores ou iguais a 1 , a região correspondente do gráfico é pintada de branco e quando ao menos o modulo de um deles extrapolar esse valor, a região é pintada de preto.

A Figura 17 traz as regiões de estabilidade para a equação de Mathieu por meio da abordagem aqui adotada. Para verificar o resultado, foram inseridas duas curvas próximas a $\delta=1$. Essas curvas representam aproximações das curvas de transição e foram obtidas através da aplicação método das múltiplas escalas à equação de Mathieu (ver Bender e Orszag (1978)).

O resultado numérico obtido através da aplicação da Teoria de Floquet na equação de Mathieu está de acordo com os mapas de estabilidade apresentados por Nayfeh e Mook (1979) e Rand (2012). Além da semelhança com os resultados apresentados na literatura a solução analítica via método das múltiplas escalas apesentada em Bender e Orszag (1978) mostra que a solução numérica está correta na região de primeira frequência de instabilidade paramétrica como mostram as retas azul e laranja na Figura 17.

\subsection{Lacunas de conhecimento}

O modelo estudado é considerado um clássico entre os problemas de escoamento interno em tubos. Os resultados obtidos por Benjamin e Païdoussis ilustram as principais literaturas especializadas no assunto. A ideia desta dissertação é adicionar o efeito de 
instabilidade paramétrica ao problema clássico do tubo de Benjamin e verificar os efeitos da solicitação combinada na resposta estrutural.

O efeito do escoamento interno em tubulações, combinado com excitação paramétrica é assunto atual de pesquisa como foi mostrado na Seção 2.3. Pelo fato de ser tema atual de estudo, a proposta deste trabalho é contribuir com o desenvolvimento no assunto, fazendo uso de uma teoria clássica para análise de sistemas não autônomos. A ideia é verificar o comportamento do modelo quando submetido aos efeitos combinados de excitação pelo escoamento interno e excitação paramétrica.

Dentre os trabalhos consultados a respeito deste assunto, não foi encontrado algum que tratasse o problema do tubo de Benjamin com instabilidade paramétrica. Será analisado o modelo com dois graus de liberdade e apresentados os diagramas das regiões de estabilidade e instabilidade da solução trivial para diferentes velocidades de escoamento interno e os mapas de amplitude de resposta de alguns casos através de estudos com o modelo matemático não linear. Também serão alterados os valores dos adimensionais de massa e observadas as consequentes mudanças das respostas.

Dentre os problemas de excitação paramétrica em tubos com escoamento axial interno, este feito pode se manifestar de diferentes maneiras. Pode ocorrer através do escoamento pulsante ou por movimento imposto no suporte, como já foi discutido na Seção 2.3. No trabalho aqui desenvolvido será considerado o problema de excitação paramétrica ocasionado pelo movimento imposto no suporte. 


\section{Modelo Matemático}

O modelo físico é baseado no problema clássico do tubo de Benjamin, porém incluindo uma excitação de suporte de modo a induzir a excitação paramétrica. Neste capítulo serão apresentados todos os detalhes e considerações utilizadas para o desenvolvimento das equações de movimento para o problema com 2 graus de liberdade ilustrados na Figura 18.

Figura 18 - Modelo estudado.

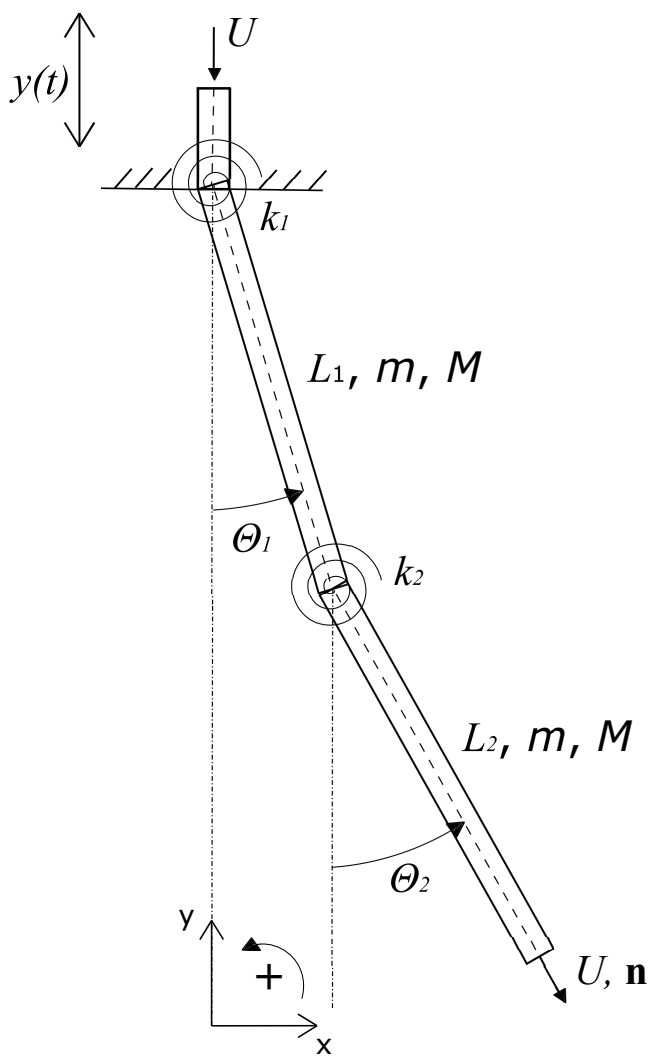

O sistema aqui estudado é compostos de tubos rígidos de comprimento $L_{1}$ e $L_{2}$, com massa por unidade de comprimento igual a $m$, diâmetro interno $d$ e diâmetro externo $D$. O conjunto está submetido a um escoamento interno uniforme e permanente com velocidade relativa aos conjunto de tubos $U$ e o fluido possui massa por unidade de comprimento $M$. As barras são conectadas por molas rotacionais lineares iguais com valores de rigidez $k_{1} \mathrm{e}$ $k_{2}$.

A parcela referente à excitação de suporte é representada na Figura 18 como $y(t)$. Essa parcela representa a variação da posição vertical do suporte no tempo e será considerada harmônica com valor igual a $y(t)=A \cos (\omega t)$, onde $A$ representa a amplitude do movimento do suporte e $\omega$ a sua frequência. 


\subsection{Trabalho virtual das forças não conservativas}

Nesta seção, será mostrado como foi obtida a parcela referente ao trabalho virtual não conservativo ocasionado pelo escoamento interno. Será utilizada a formulação a partir do princípio de conservação da massa e o resultado poderá ser utilizado no modelo de dois graus de liberdade.

Para encontrar as equações de movimento é necessário estudar quais os efeitos que são causados na estrutura pelo escoamento interno. De forma a tornar isso possível, foi utilizado o teorema do transporte de Reynolds, uma ferramenta adequada para representação de sistemas em volumes de controle.

Um sistema representa uma quantidade de material com massa fixa que pode ser identificada em qualquer instante. Por sua vez, o volume de controle é uma figura geométrica pré-estabelecida no espaço onde o fluido escoa através dela. Os conceitos citados anteriormente são importantes para identificar as forças que são aplicadas pelo fluido na estrutura. A Figura 19 apresenta o volume de controle considerado e a partir dele serão expostas uma série de considerações e simplificações.

Figura 19 - Volume de controle.

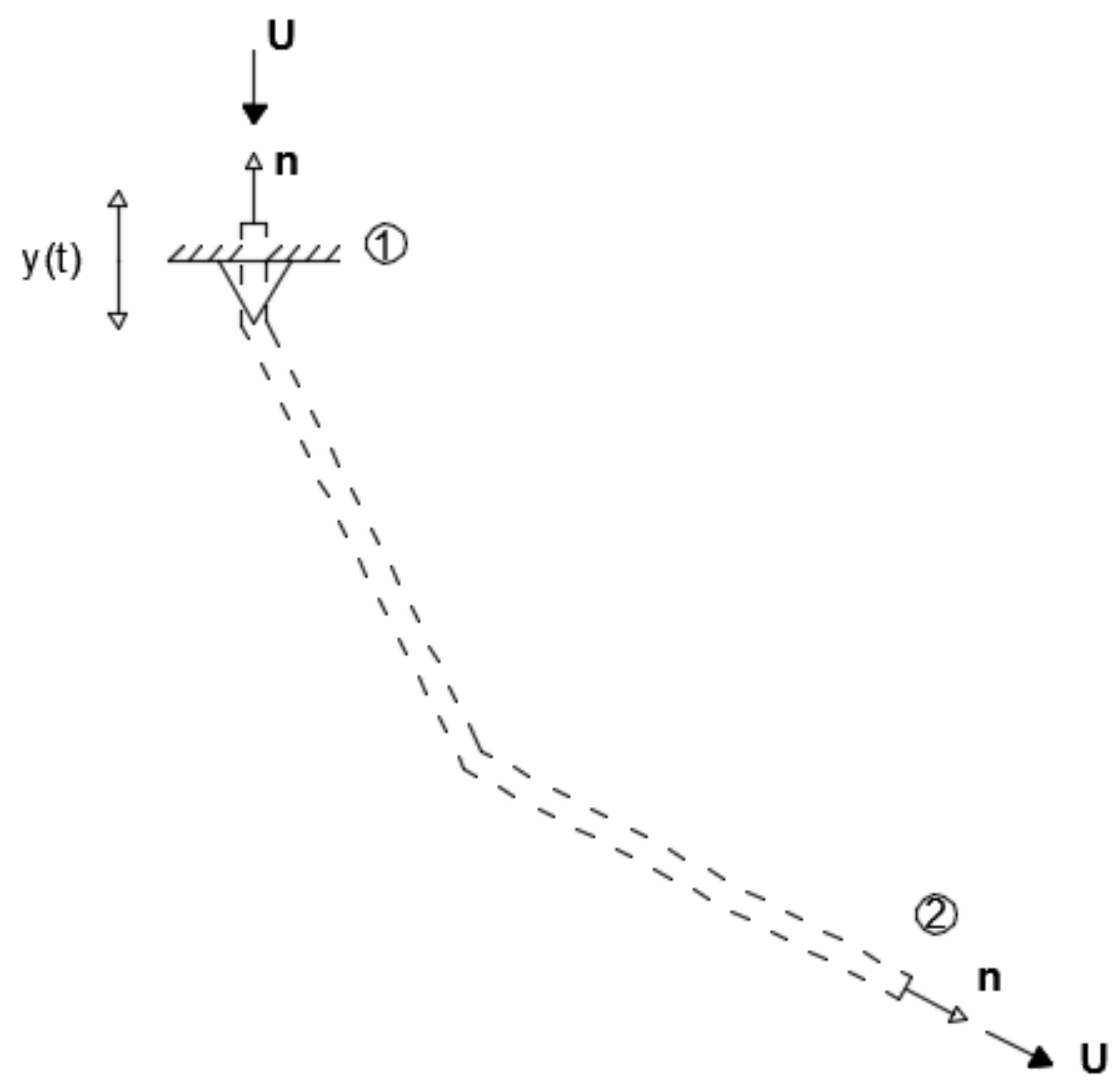

Aplicando o teorema de transporte de Reynolds no volume de controle estudado é 
possível obter a seguinte equação:

$$
\begin{gathered}
\frac{D}{D t} \int_{s i s t} \rho d V=\frac{\partial}{\partial t} \int_{v c} \rho d V+\int_{s c} \rho \mathbf{V}_{t o t} \cdot \mathbf{n} d S \\
\mathbf{V}_{t o t}=\mathbf{V}_{v c}+\mathbf{U}
\end{gathered}
$$

A Equação 3.1 representa a taxa de variação temporal de massa do sistema analisado. Esta taxa de variação temporal de massa do sistema é expressa pela soma da taxa de variação temporal de massa contida no volume de controle com a vazão líquida de massa que atravessa as superfícies de controle.

Já a Equação 3.2 descreve a velocidade absoluta $\mathbf{V}_{\text {tot }}$ como sendo a soma entre a velocidade do escoamento interno $\mathbf{U}$ medida em relação a um observador solidário ao volume de controle e a velocidades do volume de controle $\mathbf{V}_{v c}$ relativa a um observador posicionado em um referencial fixo. Neste caso o volume de controle foi definido de forma a se confundir com o conjunto de tubos.

Para o sistema estudado será considerado escoamento permanente, portanto, o primeiro termo do lado direito da Equação 3.1 pode ser considerado nulo pois a taxa de variação temporal de massa do sistema é igual a zero, desta forma:

$$
\frac{D}{D t} \int_{s i s t} \rho d V=\int_{s c 1} \rho \mathbf{V}_{t o t} \cdot \mathbf{n} d S+\int_{s c 2} \rho \mathbf{V}_{t o t} \cdot \mathbf{n} d S
$$

Onde as superfícies $s c 1$ e $s c 2$ são definidas como os limites de integração na Equação 3.3 e podem ser vistas na Figura 19. Mais especificamente, sc1 representa a superfície de entrada do escoamento e $s c 2$ a de saída. A segunda lei de Newton para sistemas define que a taxa de variação temporal da quantidade de movimento do sistema é igual a soma das forças externas que atuam sobre ele, conforme mostra a Equação 3.4.

$$
\sum F_{\text {sis }}=\frac{D}{D t} \int_{\text {sist }} \rho \mathbf{V}_{\text {tot }} d V
$$

Combinando a Segunda Lei de Newton para sistemas de controle (ver, Equação 3.4) com os resultados encontrados nas Equações 3.3 chega-se na Equação 3.5.

$$
\sum F_{s i s}=\int_{s c 1} \mathbf{V}_{t o t} \rho \mathbf{V}_{t o t} \cdot \mathbf{n} d S+\int_{s c 2} \mathbf{V}_{t o t} \rho \mathbf{V}_{t o t} \cdot \mathbf{n} d S
$$

Combinando as Equações 3.2 e 3.5 é possível encontrar a Equação 3.6. Resolvendo a Equação 3.6 e lembrando que $\rho d S=M$, onde $\mathrm{M}$ é a massa do fluido por unidade de 
comprimento, chega-se a Equação 3.7.

$$
\begin{gathered}
\sum F_{s i s}=\int_{s c 1}\left(\mathbf{V}_{v c}+\mathbf{U}\right) \rho \mathbf{U} \cdot \mathbf{n} d S+\int_{s c 2}\left(\mathbf{V}_{v c}+\mathbf{U}\right) \rho \mathbf{U} \cdot \mathbf{n} d S \\
\sum F_{s i s}=-\left[\dot{\mathbf{r}}_{0}+U \mathbf{n}_{0}\right] U M+\left[\dot{\mathbf{r}}_{L}+U \mathbf{n}_{L}\right] U M
\end{gathered}
$$

Na Equação 3.7 a parcela de velocidade referente ao movimento do volume de controle, $\mathbf{V}_{v c}$, que pode ser confundida com a velocidade com que se movimentam os tubos. As velocidades de movimentação das extremidades dos tubos são apresentadas como $\dot{\mathbf{r}}_{0} \mathrm{e}$ $\dot{\mathbf{r}}_{L}$ respectivamente. Os vetores de velocidade $\dot{\mathbf{r}}_{0}$ e $\dot{\mathbf{r}}_{L}$ são obtidos através da derivada dos vetores das posições $\mathbf{r}_{0}$ e $\mathbf{r}_{L}$ no tempo.

Além desses esforços, também existem as forças de pressão que podem ser vistas na Figura 20 e as forças de atrito entre o fluido e a tubulação. As forças de atrito foram desprezadas no presente estudo, dado que a hipótese de $p l u g$-flow ${ }^{1}$ é adotada.

Figura 20 - Forças de pressão.

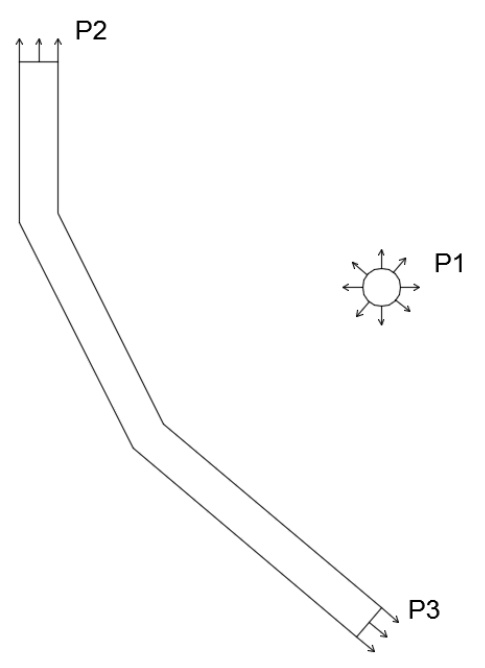

Todas as forças obtidas até o momento serão importantes na determinação da parcela do trabalho virtual das forças não conservativas, uma vez que, essas são primordiais para que posteriormente seja possível encontrar as equações de movimento do sistema em estudo. A parcela do trabalho virtual das forças não conservativas pode ser vista na Equação 3.8.

$$
\delta W_{n}=\left(P_{1} \frac{\partial \mathbf{r}}{\partial \theta_{n}}+P_{2} \frac{\partial \mathbf{r}_{0}}{\partial \theta_{n}}+P_{3} \frac{\partial \mathbf{r}_{L}}{\partial \theta_{n}}-\left[\dot{\mathbf{r}}_{0}+U \mathbf{n}_{0}\right] U M \frac{\partial \mathbf{r}_{0}}{\partial \theta_{n}}+\left[\dot{\mathbf{r}}_{L}+U \mathbf{n}_{L}\right] U M \frac{\partial \mathbf{r}_{L}}{\partial \theta_{n}}\right) \cdot \delta \theta_{n}
$$

1 O modelo de plug-flow considera o fluido como sendo um cilindro deformável que escoa por dentro da tubulação com velocidade constante ao longo da seção transversal. Fisicamente, não há formação de camada limite na parede interna do tubo. 
Observando a Equação 3.8 e a Figura 20, é possível definir $P_{1}$ como sendo a pressão radial que o fluido aplica na tubulação, $P_{2}$ a pressão na superfície de entrada e $P_{3}$ na saída. Os vetores $\mathbf{r}_{0}$ e $\mathbf{r}_{L}$ podem ser vistos na Figura 21 .

Figura 21 - Vetores $\mathbf{r}_{0}$ e $\mathbf{r}_{L}$.

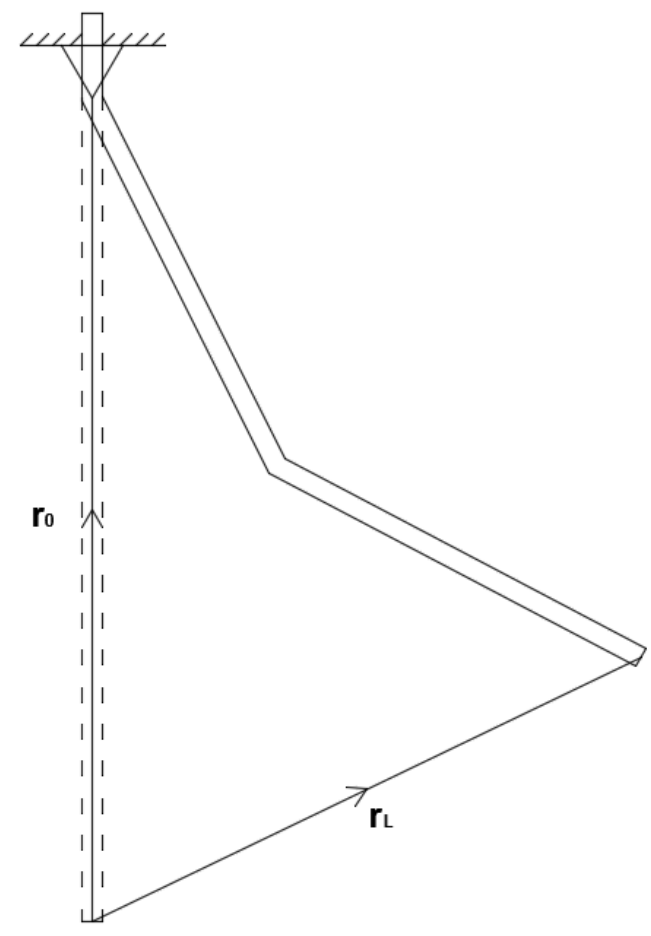

Analisando todos os termos da Equação 3.8 é possível concluir que $P_{1}$ não realiza trabalho pois a variação do diâmetro é desprezível e a parcela de $P_{2}$ é diferente de zero, mas $\partial r_{0} / \partial \theta_{n}$ é zero portanto também não realiza trabalho. Como está sendo considerado o valor da pressão relativo à pressão atmosférica, $P_{3}$ é igual a zero não realizando trabalho também. O motivo pelo qual o quarto termo pode ser desprezado é o mesmo que foi considerado no caso da pressão $P_{2}$, restando apenas a última parcela.

$$
\delta W_{n}=\left(\left[\dot{\mathbf{r}}_{L}+U \mathbf{n}_{L}\right] U M \frac{\partial \mathbf{r}_{L}}{\partial \theta_{n}}\right) \cdot \delta \theta_{n}
$$

Este resultado pode ser verificado em McIver (1973), que encontrou a resposta a partir do principio de Hamilton. A equação obtida será inclusa na equação de EulerLagrange nas próximas seções para que as equações de movimento sejam deduzidas.

\subsection{Parâmetros de rigidez e amortecimento}

O modelo estudado possui molas rotacionais que simulam os efeitos de rigidez flexional do tubo e amortecedores que representam a dissipação de energia por parte da 
estrutura. Esses parâmetros que precisam ter seus valores determinados. Para o coeficiente de rigidez, foram arbitradas as propriedades físicas e geométricas de um tubo para que os elementos pontuais de rigidez pudessem ser calculados. No caso do coeficiente de amortecimento, foi escolhido o modelo proporcional de Rayleigh. Essa escolha foi feita tendo em vista a conveniência em variar os valores da taxa de amortecimento estrutural ao invés dos coeficientes de amortecimento. As próximas subseções detalham os procedimentos de cálculos dos valores de rigidez e amortecimento.

\subsubsection{Determinação dos coeficientes de rigidez}

O coeficiente de rigidez deve ser cuidadosamente escolhido pois pode mudar completamente o comportamento do sistema estudado. De forma a tentar aproximar o comportamento do modelo proposto ao de um sistema contínuo, os valores adotados para os coeficientes de rigidez $k_{1}$ e $k_{2}$ a serem empregados nas conexões da cadeia de tubos foram baseados na frequência natural do primeiro modo de vibrar de uma viga em balanço e desconsiderando os efeitos gravitacionais.

Com a intenção de simplificar o problema, os coeficientes de rigidez $k_{1}$ e $k_{2}$ serão tratados como se possuíssem o mesmo valor $k$. Blevins (1984) apresenta a solução analítica das frequências naturais e modos de vibrar para o problema da viga em balanço, sem escoamento axial, na posição horizontal e sem excitação paramétrica na forma da Equação 3.10 .

$$
f_{i}=\frac{\bar{\lambda}_{i}^{2}}{\hat{L}^{2}}\left(\frac{E_{0} I}{\bar{m}}\right)^{1 / 2} \text { onde } \mathrm{i}=1,2 \ldots \mathrm{n}
$$

$\mathrm{Na}$ Equação 3.10, $f_{i}$ é valor da frequência natural em $\mathrm{rad} / \mathrm{s}$, do i-ésimo modo de vibrar. $\bar{\lambda}_{i}$ é a constante que define o valor da frequência e também está disponível em Blevins (1984), sendo função das condições de contorno. $\hat{L}$ é o comprimento total do conjunto de tubos, $\bar{m}$ é a massa por unidade de comprimento da tubulação ${ }^{2}$ e $E_{0} I$ representa a rigidez flexional.

Para encontrar os valores dos coeficientes de rigidez $k_{i}$ que representam o problema do tubo contínuo, é necessário igualar as frequências naturais dos dois primeiros modos obtidos através da Equação 3.10 com os que foram calculados numericamente para o sistema estudado (ver Figura 18).

Algumas simplificações foram adotadas para a realização dos cálculos, dentre elas:

1. Foi desprezado o efeito gravitacional posicionando o conjunto de tubos na horizontal;

2 Para o problema em estudo, a massa por unidade de comprimento do sistema envolverá tanto o termo estrutural quanto o termo devido ao fluido. 
2. O movimento imposto no suporte foi desprezado nesta etapa;

3. Não foi considerado o escoamento axial no conjunto, apenas o fluido em repouso no interior do tubo.

Igualando as duas primeiras frequências naturais do sistema contínuo com as do discreto proposto, chega-se a uma equação em função de $k$. Substituindo os valores das variáveis apresentadas na Tab. 1 e resolvendo a equação numericamente é possível encontrar a relação que define o valor da constante de rigidez (ver, Equação 3.11).

$$
k=\frac{4,5335 E_{0} I}{\hat{L}}
$$

Tabela 1 - Tubo escolhido para o cálculo dos coeficientes de amortecimento e rigidez.

\begin{tabular}{ll|l}
\multicolumn{3}{c}{ Propriedades do tubo } \\
\hline Comprimento do tubo & $\hat{L}[\mathrm{~m}]$ & 20,0 \\
Momento de Inércia & $\mathrm{I}\left[\mathrm{cm}^{4}\right]$ & 43,29 \\
Módulo de Elasticidade & $E_{0}[\mathrm{MPa}]$ & 1100 \\
Massa/comp. tubo & $\mathrm{m}[\mathrm{kg} / \mathrm{m}]$ & 0,307 \\
Massa/comp. fluido & $\mathrm{M}[\mathrm{kg} / \mathrm{m}]$ & 0,921 \\
Frequência natural 1 $1^{\mathrm{o}}$ modo & $\hat{\omega}_{1}[\mathrm{rad} / \mathrm{s}]$ & 0,88 \\
Frequência natural 2 $2^{\mathrm{o}}$ modo & $\hat{\omega}_{2}[\mathrm{rad} / \mathrm{s}]$ & 2,95 \\
Constante & $\bar{\lambda}_{1}$ & 1,8751 \\
Constante & $\bar{\lambda}_{2}$ & 4,6941
\end{tabular}

Em Orsino e Giulio (2019) foi proposta uma metodologia utilizando quociente de Rayleigh para calibrar a constante $k$. Afim de verificar a relação apresentada na Equação 3.11 foi utilizada essa segunda alternativa, matematicamente descrita pela Equação 3.12. Para a sequência deste trabalho será adotado o resultado apresentado na Equação 3.11.

$$
k=\frac{4,4774 E_{0} I}{\hat{L}}
$$

\subsubsection{Determinação dos coeficientes de amortecimento}

O valor da constante de amortecimento foi determinado a partir do valor da taxa de amortecimento (que pode variar, neste trabalho, entre $0 \% \leq \zeta \leq 10 \%$ ). Para o modelo de um grau de liberdade, essa relação é encontrada de forma direta utilizando a Equação 3.13 , onde $\zeta$ representa a taxa de amortecimento, $m$ é o termo inercial da equação de movimento e $\hat{\omega}$ a frequência natural não amortecida.

$$
c=2 \zeta m \hat{\omega}
$$


Para o modelo de dois graus de liberdade, foi adotado o amortecimento proporcional de Rayleigh e que define a matriz de amortecimento como sendo a combinação linear das matrizes de massa e de rigidez. Esse método permite encontrar valores para os coeficientes de amortecimento a partir das taxas de amortecimento estrutural. O modelo matemático para cálculo do amortecimento proporcional foi extraído de Mazzilli et al. (2016) e poderá ser visto na Equação 3.14 .

O amortecimento proporcional foi calculado para o modelo estudado. Neste caso, a velocidade do escoamento interno é nula, o suporte é fixo e a massa de fluido foi somada a massa dos tubos.

$$
\left[\begin{array}{l}
a_{0} \\
a_{1}
\end{array}\right]=2 \frac{\hat{\omega}_{1} \hat{\omega}_{2}}{\hat{\omega}_{2}^{2}-\hat{\omega}_{1}^{2}}\left[\begin{array}{cc}
\hat{\omega}_{2} & -\hat{\omega}_{1} \\
-1 / \hat{\omega}_{2} & 1 / \hat{\omega}_{1}
\end{array}\right]\left[\begin{array}{l}
\zeta_{1} \\
\zeta_{2}
\end{array}\right]
$$

Na Equação 3.14, $a_{0}$ e $a_{1}$ correspondem aos termos que multiplicam as matrizes de massa e de rigidez, respectivamente. As frequências naturais $\hat{\omega}_{2}$ e $\hat{\omega}_{1}$ são as frequências naturais do segundo e primeiro modo respectivamente. As taxas de amortecimento do segundo e do primeiro modo são representadas como $\zeta_{2}$ e $\zeta_{1}$. Com a intenção de simplificar o problema as taxas de amortecimento tanto para o primeiro como para o segundo modo serão tratadas como se possuíssem o mesmo valor $\zeta$.

Tendo calculado os valores de $a_{0}$ e $a_{1}$ é possível encontrar a matriz proporcional de amortecimento $\mathbf{C}_{\text {est }}$. Esta é obtida de acordo com a relação apresentada na Equação 3.15. Nessa equação, $\hat{\mathbf{M}}$ e $\hat{\mathbf{K}}$ representam as matrizes de massa e rigidez linearizadas para o problema de pêndulo duplo considerando os tubos preenchidos com fluido mas sem ejeção e também sem o movimento vertical imposto nos suporte.

$$
\mathbf{C}_{e s t}=a_{0} \hat{\mathbf{M}}+a_{1} \hat{\mathbf{K}}=\left[\begin{array}{ll}
c_{1,1}^{s} & c_{1,2}^{s} \\
c_{2,1}^{s} & c_{2,2}^{s}
\end{array}\right]
$$

\subsection{Modelo 2 graus de liberdade}

Já tendo definido os valores dos coeficientes de rigidez $k$ e amortecimento $c$ (ver, Seções 3.2.1 e 3.2.2), nesta seção serão apresentadas as equações de movimento não lineares e linearizadas para o problema estudado (ver Figura 22). Também serão apresentadas as equações adimensionalizadas respeitando os adimensionais clássicos de cada um dos problemas; excitação paramétrica e tubo de Benjamin.

Para esse modelo, a energia cinética total é a somas das parcelas de energia cinética dos tubos e do conteúdo fluido existente em cada um deles. A Equação 3.16 descreve a energia cinética total do conjunto de tubos ejetando fluido. 
Figura 22 - Modelo 2 graus de liberdade.
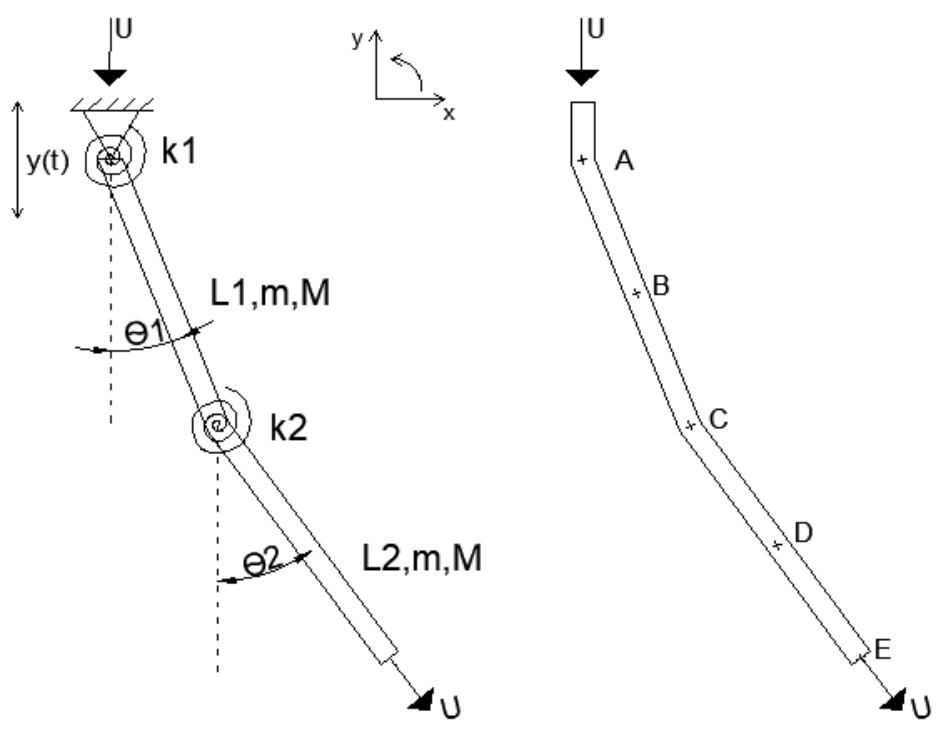

$$
\begin{aligned}
& T=\left[\frac{1}{6} L_{1}^{3}(m+M)+\frac{1}{2} L_{1}^{2} L_{2}(m+M)\right] \dot{\theta}_{1}^{2}+\frac{1}{6} L_{2}^{3}(m+M) \dot{\theta}_{2}^{2}+\frac{1}{2}\left(L_{1}+L_{2}\right)(m+M) \dot{y}^{2}- \\
& -\left(\frac{1}{2} L_{1}^{2}+L_{1} L_{2}\right)(m+M) \sin \theta_{1} \dot{\theta} \dot{y}-\frac{1}{2} L_{2}^{2} \sin \theta_{2} \dot{\theta}_{2} \dot{y}+\frac{1}{2} L_{1} L_{2}^{2}(m+M) \cos \left(\theta_{2}-\theta_{1}\right) \dot{\theta}_{1} \dot{\theta}_{2}+ \\
& +\left(L_{1}+L_{2}\right) M U \cos \theta_{2} \dot{y}+L_{1} L_{2} M U \sin \left(\theta_{2}-\theta_{1}\right) \dot{\theta}_{1}+\frac{1}{2}\left(L_{1}+L_{2}\right) M U^{2}
\end{aligned}
$$

A parcela referente à energia potencial total é a soma das parcelas que correspondem a energia potencial elástica e gravitacional das molas rotacionais, dos tubos e do fluido. A Equação 3.17 representa a energia potencial total.

$$
\begin{aligned}
& E=L_{2}(m+M) g\left(L_{1}+\frac{L_{2}}{2}-L_{1} \cos \theta_{1}-\frac{L_{2}}{2} \cos \theta_{2}-y\right)+\frac{1}{2} k_{2}\left(\theta_{2}-\theta_{1}\right)^{2}+\frac{1}{2} k_{1} \theta_{1}^{2}+ \\
& +L_{1}(m+M) g\left(\frac{L_{1}}{2}-\frac{L_{1}}{2} \cos \theta_{1}-y\right)
\end{aligned}
$$

Os vetores $\dot{\mathbf{r}}_{L}$ e $\mathbf{n}$ podem ser observados nas Equações 3.18 e 3.19.

$$
\begin{gathered}
\dot{\mathbf{r}}_{L}=\left[L_{1} \cos \theta_{1} \dot{\theta}_{1}+L_{2} \cos \theta_{2} \dot{\theta}_{2} ; L_{1} \sin \theta_{1} \dot{\theta}_{1}+L_{2} \sin \theta_{2} \dot{\theta}_{2}-\dot{y} ; 0\right] \\
\mathbf{n}=\left[\sin \theta_{2} ;-\cos \theta_{2} ; 0\right]
\end{gathered}
$$

Com todas as informações apresentadas anteriormente e as Equações 3.20, é possível encontrar as equações não lineares de movimento, dadas pela Equação 3.21. 


$$
\begin{aligned}
& \frac{d}{d t}\left(\frac{\partial L}{\partial \dot{\theta}_{1}}\right)-\frac{\partial L}{\partial \theta_{1}}+M U\left[\dot{\mathbf{r}}_{L}+U \mathbf{n}\right] \frac{\partial \mathbf{r}_{L}}{\partial \theta_{1}}+\sum_{i=1}^{2} c_{i, 1}^{s} \dot{\theta}_{1}=0 \\
& \frac{d}{d t}\left(\frac{\partial L}{\partial \dot{\theta}_{2}}\right)-\frac{\partial L}{\partial \theta_{2}}+M U\left[\dot{\mathbf{r}}_{L}+U \mathbf{n}\right] \frac{\partial \mathbf{r}_{L}}{\partial \theta_{2}}+\sum_{i=1}^{2} c_{i, 2}^{s} \dot{\theta}_{2}=0
\end{aligned}
$$

As equações não lineares encontradas podem ser comparadas com aquelas obtidas por Païdoussis e que foram replicadas nas Equações 2.3 e 2.4. As diferenças entre essas equações e as que foram aqui obtidas são os termos referentes ao movimento imposto no suporte $\ddot{y}$, as parcelas do amortecimento proporcional e o termo que considera o escoamento variável no tempo $M L_{1} L_{2} \dot{U} \sin \left(\theta_{2}-\theta_{1}\right)$, lembrando que a hipótese de escoamento constante no tempo foi adotada no modelo aqui proposto.

Além das diferenças conceituais, foi verificado que o termo $\left(\frac{1}{2}(M+m) g L_{2} \sin \theta_{2}\right)$ presente na equação retirada de Païdoussis (1998) possui um equivoco dimensional, provavelmente devido a um erro de digitação. Este termo deveria ser escrito da seguinte forma para garantir a correção da unidade de momento $\left(\frac{1}{2}(M+m) g L_{2}^{2} \sin \theta_{2}\right)$.

As equações não lineares de movimento podem ser vistas no formato matricial apresentado na Equação 3.21.

$$
\mathbf{M} \ddot{\theta}+\mathbf{C} \dot{\theta}+\mathbf{K} \theta=\mathbf{F}
$$

onde $\theta=\left\{\begin{array}{ll}\theta_{1} & \theta_{2}\end{array}\right\}^{T}$ e:

$$
\begin{aligned}
& \mathbf{M}=\left[\begin{array}{cc}
\frac{1}{3} L_{1}^{3}(m+M)+L_{1}^{2} L_{2}(m+M) & \frac{1}{2} L_{1} L_{2}^{2}(m+M) \cos \left(\theta_{2}-\theta_{1}\right) \\
\frac{1}{2} L_{1} L_{2}^{2}(m+M) \cos \left(\theta_{2}-\theta_{1}\right) & \frac{1}{3} L_{2}^{3}(m+M)
\end{array}\right] \\
& \mathbf{C}=\left[\begin{array}{cc}
c_{1,1}^{s} & c_{1,2}^{s} \\
c_{2,1}^{s} & c_{2,2}^{s}
\end{array}\right]+\left[\begin{array}{cc}
L_{1}^{2} M U & L_{1} L_{2} M U \cos \left(\theta_{2}-\theta_{1}\right) \\
L_{1} L_{2} M U \cos \left(\theta_{2}-\theta_{1}\right) & L_{2}^{2} M U
\end{array}\right]+ \\
& +\left[\begin{array}{cc}
0 & L_{1} L_{2} M U \cos \left(\theta_{2}-\theta_{1}\right) \\
-L_{1} L_{2} M U \cos \left(\theta_{2}-\theta_{1}\right) & 0
\end{array}\right]+ \\
& +\left[\begin{array}{cc}
0 & -\frac{1}{2} L_{1} L_{2}^{2}(m+M) \sin \left(\theta_{2}-\theta_{1}\right) \dot{\theta_{2}} \\
\frac{1}{2} L_{1} L_{2}^{2}(m+M) \sin \left(\theta_{2}-\theta_{1}\right) \dot{\theta}_{1} & 0
\end{array}\right] \\
& \mathbf{K}=\left[\begin{array}{cc}
k_{1}+k_{2} & -k_{2} \\
-k_{2} & k_{2}
\end{array}\right] \\
& \mathbf{F}=\left\{\begin{array}{c}
L_{1} M U^{2} \sin \left(\theta_{2}-\theta_{1}\right)+\left[\frac{1}{2} L_{1}^{2}(m+M)+L_{1} L_{2}(m+M)\right](g-\ddot{y}) \sin \theta_{1} \\
\frac{1}{2} L_{2}^{2}(m+M)(g-\ddot{y}) \sin \theta_{2}
\end{array}\right\}
\end{aligned}
$$




$$
\begin{aligned}
& c_{1,1}^{s}=a_{0} \omega(m+M)\left(\frac{L_{1}^{3}}{3}+L_{1}^{2} L_{2}\right)+\frac{a_{1}\left(k_{1}+k_{2}+(m+M) g\left(L_{1} L_{2}+\frac{L_{1}^{2}}{2}\right)\right)}{\omega} \\
& c_{1,2}^{s}=c_{2,1}^{s}=\frac{1}{2} a_{0} \omega L_{1} L_{2}^{2}(m+M)-\frac{a_{1} k_{2}}{\omega} \\
& c_{2,2}^{s}=a_{0} \omega(m+M)\left(\frac{L_{2}^{3}}{3}\right)+\frac{a_{1}\left(k_{2}+(m+M) g\left(\frac{L_{2}^{2}}{2}\right)\right)}{\omega}
\end{aligned}
$$

Perceba que a matriz de amortecimento $\mathbf{C}$ pode ser escrita como a soma de quatro parcelas. A primeira delas definida pelos coeficientes $c_{i, j}^{s}$, está relacionada ao amortecimento estrutural. A segunda representa a parcela de forças generalizadas simétricas dissipativas. A terceira é assimétrica e corresponde a parcela de forças generalizadas giroscópicas. A quarta é assimétrica também e está associada aos termos de forças centrífugas.

A matriz relacionada ao amortecimento estrutural teve seus termos calculados adotando a premissa de que a taxa de amortecimento $\zeta$ (assumida na presente análise como sendo igual para os dois modos de vibração $\zeta_{1}=\zeta_{2}=\zeta$ ) é o parâmetro de entrada para a obtenção das constantes $a_{0}$ e $a_{1}$ conforme foi mostrado na Seção 3.2.2.

Finalmente, é importante pontuar que o vetor $\mathbf{F}$ contém termos associados com forças centrifugas (proporcional a $U^{2}$ ) e forças gravitacionais. Para a análise de estabilidade, o modelo matemático é linearizado em torno da configuração trivial de equilíbrio $\left(\theta_{1}=\right.$ $\theta_{2}=0$ ), de acordo com a Equação 3.22 .

$$
\overline{\mathbf{M}} \ddot{\theta}+\overline{\mathbf{C}} \dot{\theta}+\overline{\mathbf{K}} \theta=\mathbf{0}
$$

onde:

$$
\begin{aligned}
& \overline{\mathbf{M}}=\left[\begin{array}{cc}
\frac{1}{3} L_{1}^{3}(m+M)+L_{1}^{2} L_{2}(m+M) & \frac{1}{2} L_{1} L_{2}^{2}(m+M) \\
\frac{1}{2} L_{1} L_{2}^{2}(m+M) & \frac{1}{3} L_{2}^{3}(m+M)
\end{array}\right] \\
& \overline{\mathbf{C}}=\left[\begin{array}{cc}
L_{1}^{2} M U+c_{1,1}^{s} & 2 L_{1} L_{2} M U+c_{1,2}^{s} \\
c_{2,1}^{s} & L_{2}^{2} M U+c_{2,2}^{s}
\end{array}\right] \\
& \overline{\mathbf{K}}=\left[\begin{array}{cc}
{\left[\frac{1}{2} L_{1}^{2}(m+M)+L_{1} L_{2}(m+M)\right](g-\ddot{y})} & 0 \\
0 & \frac{1}{2} L_{2}^{2}(m+M)(g-\ddot{y})
\end{array}\right]+ \\
& +\left[\begin{array}{cc}
-L_{1} M U^{2} & \frac{1}{2} L_{1} M U^{2} \\
\frac{1}{2} L_{1} M U^{2} & 0
\end{array}\right]+\left[\begin{array}{cc}
0 & \frac{1}{2} L_{1} M U^{2} \\
-\frac{1}{2} L_{1} M U^{2} & 0
\end{array}\right]+\left[\begin{array}{cc}
k_{1}+k_{2} & -k_{2} \\
-k_{2} & k_{2}
\end{array}\right]
\end{aligned}
$$


No modelo matemático linearizado, a matriz de rigidez pode ser expressa como a soma de quatro parcelas. A primeira parcela está associada com a força gravitacional, corrigida pela aceleração do movimento imposto ao suporte. A segunda e a terceira parcelas representam as parcelas simétrica e assimétrica associadas às forças inerciais e aquelas provenientes do escoamento interno respectivamente. Finalmente, a quarta parcela representa à rigidez flexional equivalente representada pelas molas rotacionais.

A ideia de separar a matriz de amortecimento para as equações não lineares e a matriz de rigidez para as equações lineares foi baseada no trabalho similar desenvolvido por Thompson (1982). É importante pontuar que no trabalho desenvolvido por Thompson (1982) não aparecem os termos associados à excitação paramétrica e ao amortecimento estrutural.

Com o intuito de generalizar a solução, o modelo matemático será reescrito na forma adimensional. Para isto, o tempo adimensional $\tau$ é definido como $\tau=\omega t$. Deste ponto em diante, ( )' representa a derivada com relação a $\tau$ e $\hat{L}=L_{1}+L_{2}$ é o comprimento total do conjunto de tubos. São definidas as quantidades adimensionais apresentadas na Equação 3.23, propostas combinando os adimensionais clássicos dos problemas do tubo de Benjamin e da equação de Mathieu exaustivamente estudados na literatura. Logo, esses adimensionais clássicos podem ser conferidos nos estudos Païdoussis (1998) e Nayfeh e Mook (1979). É importante salientar ao leitor que $\delta$ e $\epsilon$ estão associados com a frequência e a amplitude do movimento imposto no suporte, respectivamente.

$$
\begin{aligned}
& \lambda=\frac{L_{2}}{\hat{L}} \quad \beta=\frac{M}{(M+m)} \quad \psi=\sqrt{\frac{E_{0} I}{g \hat{L}^{3}(m+M)}} \\
& K_{i}=\frac{k_{i} \hat{L}}{E_{0} I} \quad \epsilon=\frac{A}{\hat{L}} \quad \delta=\frac{g}{\omega^{2} \hat{L}} \quad u=U \hat{L} \sqrt{\frac{M}{E_{0} I}}
\end{aligned}
$$

Note que $\lambda$ representa o adimensional de comprimento do segundo tubo, $\beta$ é o parâmetro adimensional de massa, $u$ é o adimensional da velocidade de escoamento interno e $K_{i}$ é o adimensional de rigidez flexional.

Usando os adimensionais definidos na Equação 3.23, as equações adimensionais não lineares de movimento são apresentadas na Equação 3.24.

$$
\mathbf{M}^{*} \theta^{\prime \prime}+\mathbf{C}^{*} \theta^{\prime}+\mathbf{K}^{*} \theta=\mathbf{F}^{*}
$$

onde: $\theta=\left\{\begin{array}{ll}\theta_{1} & \theta_{2}\end{array}\right\}^{T}$

$$
\mathbf{M}^{*}=\left[\begin{array}{cc}
\left(1-\lambda^{2}\right)\left(\frac{1+2 \lambda}{3}\right) & \frac{1}{2} \lambda^{2}(1-\lambda) \cos \left(\theta_{2}-\theta_{1}\right) \\
\frac{1}{2} \lambda^{2}(1-\lambda) \cos \left(\theta_{2}-\theta_{1}\right) & \frac{1}{3} \lambda^{3}
\end{array}\right]
$$




$$
\begin{gathered}
\mathbf{C}^{*}=\left[\begin{array}{cc}
u \psi(1-\lambda)^{2} \sqrt{\beta \delta} & u \psi \lambda(1-\lambda) \sqrt{\beta \delta} \cos \left(\theta_{2}-\theta_{1}\right) \\
u \psi \lambda(1-\lambda) \sqrt{\beta \delta} \cos \left(\theta_{2}-\theta_{1}\right) & u \psi \lambda^{2} \sqrt{\beta \delta}
\end{array}\right]+ \\
+\left[\begin{array}{cc}
0 & u \psi \lambda(1-\lambda) \sqrt{\beta \delta} \cos \left(\theta_{2}-\theta_{1}\right) \\
-u \psi \lambda(1-\lambda) \sqrt{\beta \delta} \cos \left(\theta_{2}-\theta_{1}\right) & 0
\end{array}\right]+ \\
+\left[\begin{array}{cc}
0 & -\frac{1}{2} \lambda^{2}(1-\lambda) \sin \left(\theta_{2}-\theta_{1}\right) \theta_{2}^{\prime} \\
\frac{1}{2} \lambda^{2}(1-\lambda) \sin \left(\theta_{2}-\theta_{1}\right) \theta_{1}^{\prime} & 0
\end{array}\right]+\left[\begin{array}{cc}
C_{1,1}^{s} & C_{1,2}^{s} \\
C_{2,1}^{s} & C_{2,2}^{s}
\end{array}\right] \\
\mathbf{K}^{*}=\delta \psi^{2}\left[\begin{array}{cc}
K_{1}+K_{2} & -K_{2} \\
-K_{2} & K_{2}
\end{array}\right] \\
\mathbf{F}_{2,2}^{s}=\frac{1}{3} a_{0} \lambda^{3}+a_{1} \delta\left(\frac{1}{2} \lambda^{2}+\psi^{2} K_{2}\right) \\
C_{1,1}^{s}=a_{0}\left(\lambda(1-\lambda)\left(u^{2} \lambda^{2} \delta \sin \left(\theta_{2}-\theta_{1}\right)+\frac{(1+\lambda)}{2}(\delta+\epsilon \cos \tau) \sin \theta_{1}\right)\right. \\
\frac{\lambda^{2}}{2}(\delta+\epsilon \cos \tau) \sin \theta_{2} \\
C_{1,2}^{s}=C_{2,1}^{s}=\frac{1}{2} a_{0} \lambda^{2}(1-\lambda)-a_{1} \delta \psi^{2} K_{2} \\
\left.\frac{1}{3}(1-\lambda)^{3}\right)+a_{1} \delta\left(\psi^{2} K_{1}+\psi^{2} K_{2}+\lambda(1-\lambda)+\frac{1}{2}(1-\lambda)^{2}\right)
\end{gathered}
$$

A correspondente forma linearizada adimensional das equações de movimento pode ser expressa conforme a Equação 3.25.

$$
\overline{\mathbf{M}}^{*} \theta^{\prime \prime}+\overline{\mathbf{C}}^{*} \theta^{\prime}+\overline{\mathbf{K}}^{*} \theta=\mathbf{0}
$$

onde:

$$
\begin{gathered}
\overline{\mathbf{M}}^{*}=\left[\begin{array}{cc}
\left(1-\lambda^{2}\right)\left(\frac{1+2 \lambda}{3}\right) & \frac{1}{2} \lambda^{2}(1-\lambda) \\
\frac{1}{2} \lambda^{2}(1-\lambda) & \frac{1}{3} \lambda^{3}
\end{array}\right] \\
\overline{\mathbf{C}}^{*}=\left[\begin{array}{cc}
u \psi(1-\lambda)^{2} \sqrt{\beta \delta}+C_{1,1}^{s} & 2 u \psi \lambda(1-\lambda) \sqrt{\beta \delta}+C_{1,2}^{s} \\
C_{2,1}^{s} & u \psi \lambda^{2} \sqrt{\beta \delta}+C_{2,2}^{s}
\end{array}\right]
\end{gathered}
$$




$$
\begin{aligned}
& \overline{\mathbf{K}}^{*}=\left[\begin{array}{cc}
\bar{K}_{11}^{*} & \bar{K}_{12}^{*} \\
\bar{K}_{21}^{*} & \bar{K}_{22}^{*}
\end{array}\right] \\
& \bar{K}_{11}^{*}=\delta \psi^{2}\left(K_{1}+K_{2}-u^{2}(1-\lambda)\right)+\left(\frac{1-\lambda^{2}}{2}\right)(\delta+\epsilon \cos \tau) \\
& \bar{K}_{12}^{*}=\delta \psi^{2}\left(u^{2}(1-\lambda)-K_{2}\right) \\
& \bar{K}_{21}^{*}=-\delta \psi^{2} K_{2} \\
& \bar{K}_{22}^{*}=\delta \psi^{2} K_{2}+\frac{1}{2} \lambda^{2}(\delta+\epsilon \cos \tau)
\end{aligned}
$$

As definições apresentadas acima indicam que a excitação paramétrica aparece na diagonal principal da matriz linearizada de rigidez $\overline{\mathbf{K}}^{*}$. Por outro lado, $\bar{K}_{21}^{*}$ e $\bar{K}_{22}^{*}$ são os termos da matriz de rigidez que não são afetados pela velocidade de escoamento interno $u$.

Nas Tabelas 2, 3 e 4 estão representados os valores numéricos dos adimensionais apresentados na Equação 3.23. Os dados apresentados nas Tabelas 2, 3 e 4 serão utilizados para a obtenção dos diagramas de estabilidade para a análise linearizada e para os mapas de amplitude pós-crítica para a análise não linear que são mostrados no Capítulo 5. 
Tabela 2 - Parâmetros adimensionais e os valores adotados para $\beta=0,200$.

Grupo de parâmetros Parâmetros $\quad$ Valores

\begin{tabular}{ccc}
\hline Geometria & $\lambda$ & 0,5 \\
\hline Problema do tubo ejetando fluido & $\beta$ & 0,200 \\
Païdoussis (1998) & $\psi$ & 0,035 \\
& $u$ & $0 ; 3,0 ; 9,0 ; 12,0 ; 13,4 ; 16,0$ \\
\hline Equação de Mathieu & $\delta$ & Varia continuamente entre 0 to 6 \\
Rand (2012) & $\epsilon$ & Varia continuamente entre 0 to 6 \\
\hline Rigidez e Amortecimento & $K_{i}$ & $K_{1}=K_{2}=4,477$ \\
\hline & $\zeta$ & $0 \% ; 2,5 \% ; 5 \% ; 10 \%$ \\
\hline
\end{tabular}

Tabela 3 - Parâmetros adimensionais e os valores adotados para $\beta=0,500$.

\begin{tabular}{ccc} 
Grupo de parâmetros & Parâmetros & Valores \\
\hline Geometria & $\lambda$ & 0,5 \\
\hline Problema do tubo ejetando fluido & $\beta$ & 0,500 \\
Païdoussis (1998) & $\psi$ & 0,055 \\
\hline Equação de Mathieu & $u$ & $0 ; 3,0 ; 9,0 ; 12,0 ; 13,4 ; 16,0$ \\
Rand (2012) & $\delta$ & Varia continuamente entre 0 to 6 \\
Rigidez e Amortecimento & $\epsilon$ & Varia continuamente entre 0 to 6 \\
\hline
\end{tabular}


Tabela 4 - Parâmetros adimensionais e os valores adotados para $\beta=0,750$.

\section{Grupo de parâmetros $\quad$ Parâmetros $\quad$ Valores}

\begin{tabular}{ccc}
\hline Geometria & $\lambda$ & 0,5 \\
\hline Problema do tubo ejetando fluido & $\beta$ & 0,750 \\
Païdoussis (1998) & $\psi$ & 0,039 \\
Equação de Mathieu & $u$ & $0 ; 3,0 ; 9,0 ; 12,0 ; 13,4 ; 16,0$ \\
Rand (2012) & $\delta$ & Varia continuamente entre 0 to 6 \\
Rigidez e Amortecimento & $\epsilon$ & Varia continuamente entre 0 to 6 \\
\hline
\end{tabular}




\section{Metodologia}

A metodologia adotada consiste em estudar via, métodos numéricos e utilizando a teoria de Floquet para o sistema linearizado, a dinâmica de um conjunto de dois tubos rígidos e articulados entre si sob excitação paramétrica e ejeção de fluido. De maneira mais específica, o estudo visa, em um primeiro momento, criar diagramas que mostrem as regiões de estabilidade e instabilidade do sistema no plano de parâmetros $(\delta ; \epsilon)$, representativos da frequência e amplitude do movimento imposto no suporte. Para o problema não linear, serão mostrados como a resposta é alterada quando parâmetros de controle do modelo matemático são modificados.

As análises não lineares e linearizadas utilizam de métodos numéricos de integração de equações diferenciais ordinárias. Para as análises não lineares, os sinais oriundos da integração numérica recebem tratamento estatístico que permitem a obtenção dos valores de média e desvio padrão das respostas. Com esses valores são criados mapas, no mesmo plano de parâmetros das análises linearizadas, que permitem avaliar a resposta em regime pós-crítico. Todos os modelos matemáticos adimensionais foram numericamente integrados utilizando a função NDSolve, nativa do software Wolfram Mathematica ${ }^{\circledR}$.

De acordo com Nayfeh e Balachandran (1995), a estabilidade das órbitas periódicas pode ser investigada através da inspeção dos autovalores $\lambda_{i}$ da matriz de monodromia, daqui em diante identificada como $\Phi$. Fica aqui definido que $\left|\lambda^{*}\right|=\max \left\{\left|\lambda_{i}\right|\right\}$ e que os casos com estabilidade assintótica (respostas limitadas) ocorrem quando $\left|\lambda^{*}\right|<1$. Respostas limitadas também são obtidas quando $\left|\lambda^{*}\right|=1$. Porém, as soluções ilimitadas são caracterizadas por $\left|\lambda^{*}\right|>1$.

A existência de soluções limitadas ou ilimitadas como função dos parâmetros de controle $(\delta ; \epsilon)$ é investigada aplicando a Teoria de Floquet ao modelo linearizado adimensional dado pela Equação 3.25. Nesse estudo, são gerados diversos diagramas combinando valores para velocidade de escoamento interno $u$, massa adimensional $\beta$ e taxa de amortecimento estrutural $\zeta$. Os diagramas de estabilidade obtidos no domínio $(\delta ; \epsilon)$ foram discretizados em 200 mil pontos. Nesse tipo de estudo as equações de movimento são integradas numericamente, onde foram adotados dois conjuntos de condições iniciais como foi mostrado na Seção 2.4. O modelo matemático (ver, Equação 3.25) foi integrado em um período $\tau_{m a ́ x}=\pi$.

A análise linear permite avaliar o comportamento do sistema na região de solução limitada utilizando valores de velocidade de escoamento axial menores que a velocidade crítica (velocidades subcríticas). Para verificar o comportamento do conjunto de tubos nas regiões de solução ilimitada e também para valores de velocidade de escoamento maiores 
que a velocidade crítica é necessário estudar as equações não lineares (ver Equação 3.24). As equações não lineares permitem avaliar o comportamento do sistema em casos de resposta pós-crítica e também mostrar o que acontece, em detalhes, nas regiões onde as respostas são consideradas ilimitadas no sistema linearizado.

A análise não linear consiste em estudar as respostas em regime pós-crítico do conjunto de tubos no plano de parâmetros $(\delta ; \epsilon)$ assim como foi feito na análise linearizada, porém, em situações fora do domínio de aplicação da análise linear. Esses mapas exibirão os valores de média $\bar{y}_{t}$ e desvio padrão $y_{t, s t d}$ da série temporal de deslocamento da extremidade livre do conjunto de tubos $y_{t}(\tau)$, facilmente obtida a partir das séries temporais $\theta_{1}(\tau)$ e $\theta_{2}(\tau)$ como mostra a Equação 4.1 .

$$
y_{t}(\tau)=1-\left(\frac{L_{1}}{\hat{L}} \cos \theta_{1}(\tau)+\frac{L_{2}}{\hat{L}} \cos \theta_{2}(\tau)\right)
$$

A análise não linear também foi realizada com o auxílio da função NDSolve implementada no software Wolfram Mathematica ${ }^{\circledR}$, utilizando $\theta_{1}(0)=\theta_{2}(0)=\pi / 180$. O modelo matemático, representado pela Equação 3.24, foi integrado durante $\tau_{\text {máx }}=3000$ e para avaliação de $\bar{y}_{t}$ e $y_{t, s t d}$ foi utilizado o intervalo $2000 \leq \tau_{\text {máx }} \leq 3000$. Os mapas de amplitude obtidos, no domínio $(\delta ; \epsilon)$, foram discretizados em 200 mil pontos. É importante ressaltar que o caráter não linear do modelo matemático pode levar a uma dependência da resposta com as condições iniciais. O estudo dessa dependência, embora interessante, não faz parte do escopo desta dissertação. 


\section{Resultados}

Para o modelo estudado, foram utilizadas as Equações 3.25 e 3.24 para as análises lineares e não lineares respectivamente. Em um primeiro momento será realizada a análise linear com o objetivo do estudo da estabilidade, foco da Seção 5.1. Os diagramas de estabilidade são discutidos variando-se os adimensionais de massa $\beta$, velocidade de escoamento interno $u$ e taxa de amortecimento estrutural $\zeta$. Na Seção 5.2 serão analisadas as respostas não lineares de movimento e apresentados os mapas de resposta pós-crítica, esses permitem avaliar os resultados nas regiões de soluções ilimitadas das análises lineares e para regime super-crítico.

\subsection{Análise Linear}

Para a análise linear do modelo proposto foi utilizada a Equação 3.25. Visando o maior entendimento por parte do leitor, as equações de movimento serão aqui reapresentadas (ver Equação 5.1), bem como os adimensionais que governam o comportamento do sistema. Os diagramas de estabilidade serão organizados em forma de matriz de figuras para proporcionar uma melhor visualização global dos parâmetros adotados. Para alguns casos específicos, os mapas serão apresentados separadamente.

$$
\begin{gathered}
\lambda=\frac{L_{2}}{\hat{L}} \quad \beta=\frac{M}{(M+m)} \quad \psi=\sqrt{\frac{E I}{g \hat{L}^{3}(m+M)}} \\
K_{i}=\frac{k_{i} \hat{L}}{E I} \quad \epsilon=\frac{A}{\hat{L}} \quad \delta=\frac{g}{\omega^{2} \hat{L}} \quad u=U \hat{L} \sqrt{\frac{M}{E I}} \\
\overline{\mathbf{M}}^{*} \theta^{\prime \prime}+\overline{\mathbf{C}}^{*} \theta^{\prime}+\overline{\mathbf{K}}^{*} \theta=\mathbf{0} \\
\overline{\mathbf{M}}^{*}=\left[\begin{array}{cc}
\left(1-\lambda^{2}\right)\left(\frac{1+2 \lambda}{3}\right) & \frac{1}{2} \lambda^{2}(1-\lambda) \\
\frac{1}{2} \lambda^{2}(1-\lambda) & \frac{1}{3} \lambda^{3}
\end{array}\right] \\
\overline{\mathbf{C}}^{*}=\left[\begin{array}{cc}
u \psi(1-\lambda)^{2} \sqrt{\beta \delta}+C_{1,1}^{s} & 2 u \psi \lambda(1-\lambda) \sqrt{\beta \delta}+C_{1,2}^{s} \\
C_{2,1}^{s} & u \psi \lambda^{2} \sqrt{\beta \delta}+C_{2,2}^{s}
\end{array}\right] \\
\overline{\mathbf{K}}^{*}=\left[\begin{array}{cc}
\bar{K}_{11}^{*} & \bar{K}_{12}^{*} \\
\bar{K}_{21}^{*} & \bar{K}_{22}^{*}
\end{array}\right]
\end{gathered}
$$




$$
\begin{aligned}
& \bar{K}_{11}^{*}=\delta \psi^{2}\left(K_{1}+K_{2}-u^{2}(1-\lambda)\right)+\left(\frac{1-\lambda^{2}}{2}\right)(\delta+\epsilon \cos \tau) \\
& \bar{K}_{12}^{*}=\delta \psi^{2}\left(u^{2}(1-\lambda)-K_{2}\right) \\
& \bar{K}_{21}^{*}=-\delta \psi^{2} K_{2} \\
& \bar{K}_{22}^{*}=\delta \psi^{2} K_{2}+\frac{1}{2} \lambda^{2}(\delta+\epsilon \cos \tau)
\end{aligned}
$$

As Figuras 23, 24 e 25 apresentam os diagramas de estabilidade para os adimensionais de massa sendo iguais a $\beta=0,200, \beta=0,500$ e $\beta=0,750$ respectivamente. Para os três valores do parâmetro $\beta$, os diagramas de estabilidade apresentados possuem mesma escala nos eixos das ordenadas e abcissas e respeitam o mesmo conjunto de parâmetros de velocidade de escoamento interno $u$ e taxa de amortecimento estrutural $\zeta$.

Como mencionado anteriormente, a análise linear é feita visando o estudo da estabilidade da solução trivial, definida como sendo aquela onde o conjunto de tubos está na posição vertical. Todos os valores de velocidade $u$ adotados na análise linear são menores do que o valores críticos de acordo com os diferentes valores do parâmetro $\beta$. Para determinar os valores de velocidade crítica $u_{c}$ foi utilizado o primeiro método de Lyapunov. Os valores calculados para velocidade crítica para cada valor de $\beta$ pode ser visto na Tabela 5.

Tabela 5 - Valores de velocidade crítica para diferentes $\beta$.

\begin{tabular}{c|c}
\multicolumn{2}{c}{ Parâmetro } \\
\hline$\beta$ & $u_{c}$ \\
\hline 0,200 & 28,10 \\
0,500 & 18,68 \\
0,750 & 15,85
\end{tabular}

Os diagramas de estabilidade apresentados nas Figuras 23, 24 e 25 mostram regiões com solução limitada, representadas por uma escala de cor, que medem o valor numérico do maior autovalor (em módulo) $\left|\lambda^{*}\right|$ de acordo com a Teoria de Floquet, como já foi explicado na Seção 2.4. Já a região preenchida pela cor branca representam os pares de parâmetros que resultaram em soluções ilimitadas, ou casos onde ao menos um autovalor da matriz de monodromia apresenta módulo superior a 1,0. 
Figura 23 - Diagrama de estabilidade para $\beta=0,200$. Os eixos das abcissas e das ordenadas ( $\delta$ e $\epsilon$, respectivamente) variam de 0 to 6 .
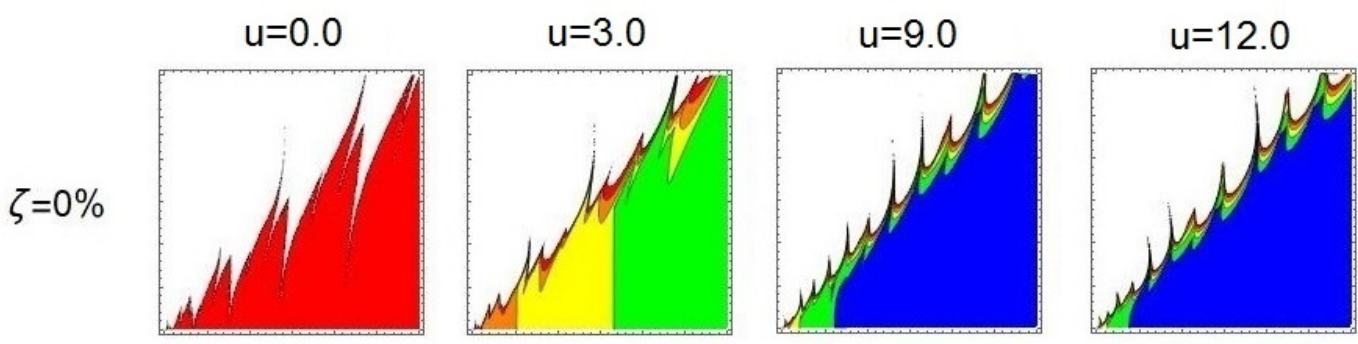

$|\lambda *|$
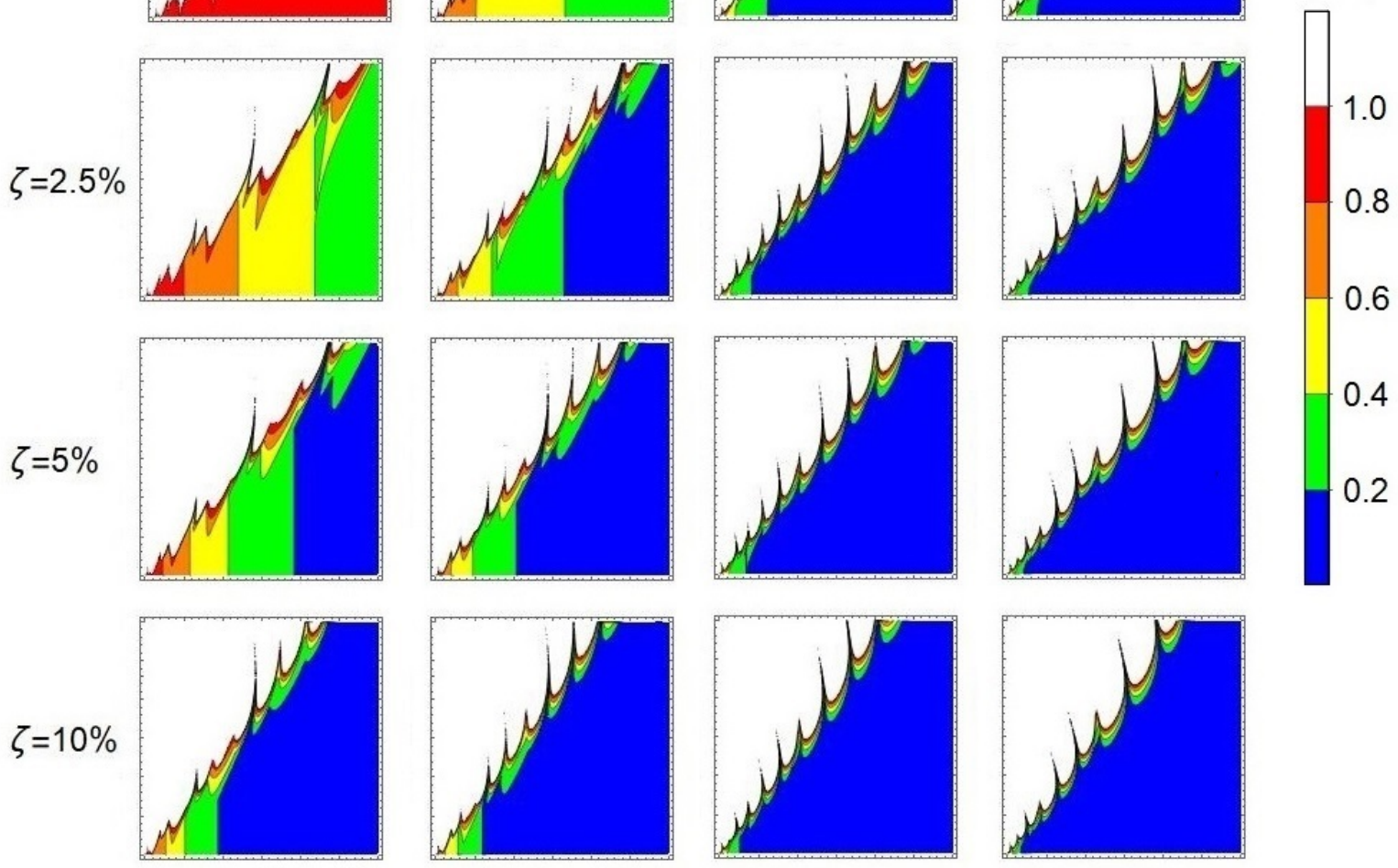

0.6
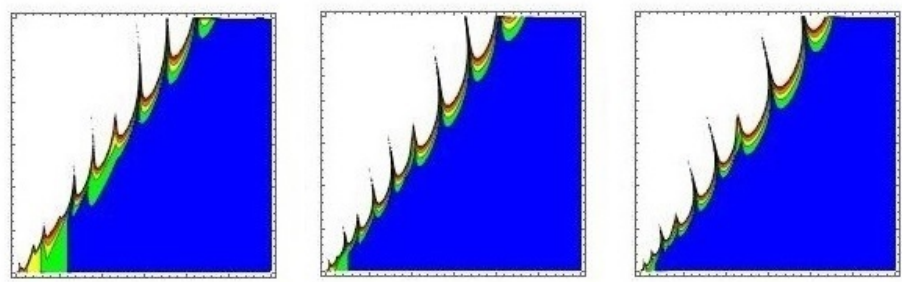
Figura 24 - Diagrama de estabilidade para $\beta=0,500$. Os eixos das abcissas e das ordenadas ( $\delta$ e $\epsilon$, respectivamente) variam de 0 to 6 .

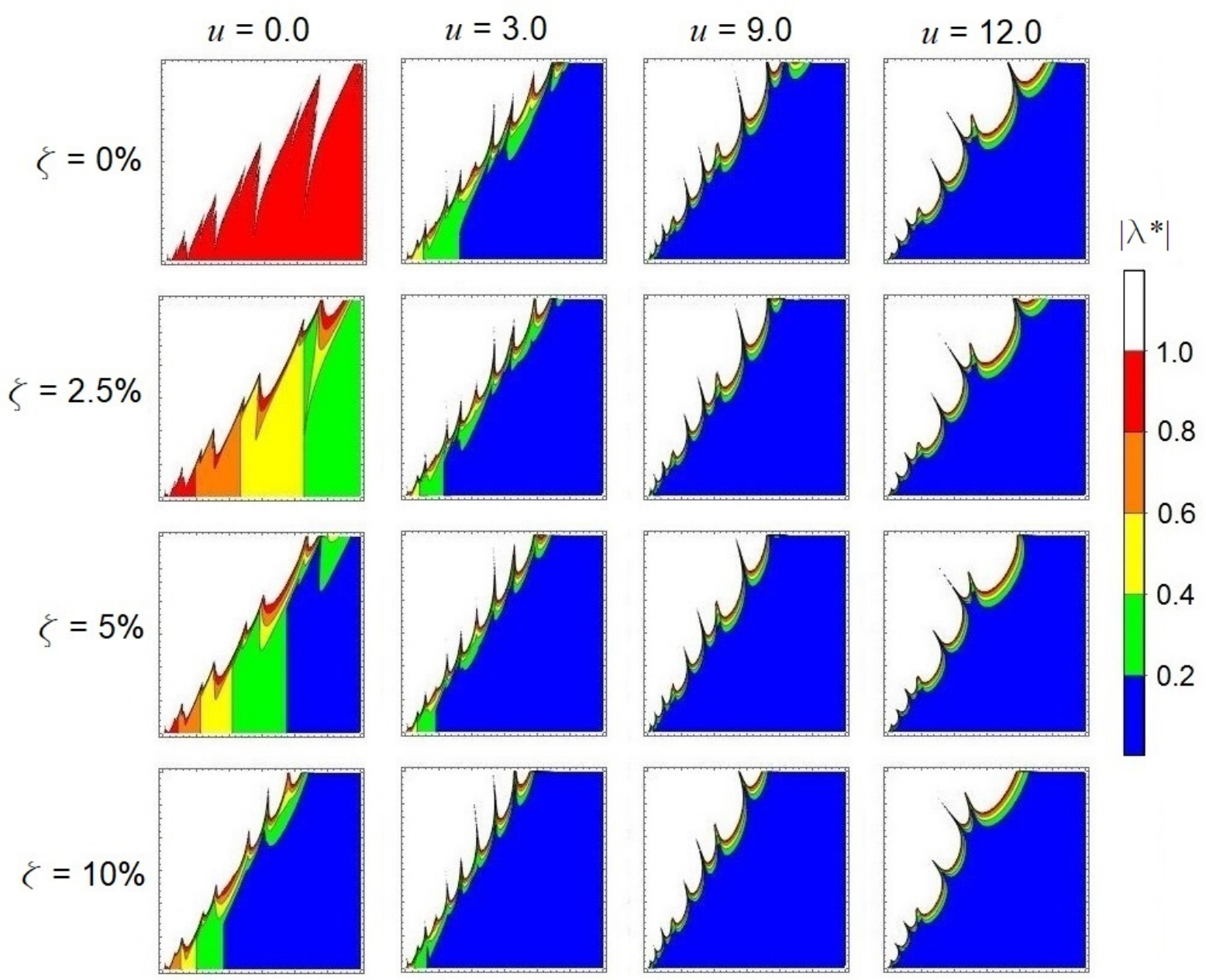


Figura 25 - Diagrama de estabilidade para $\beta=0,750$. Os eixos das abcissas e das ordenadas ( $\delta$ e $\epsilon$, respectivamente) variam de 0 to 6 .
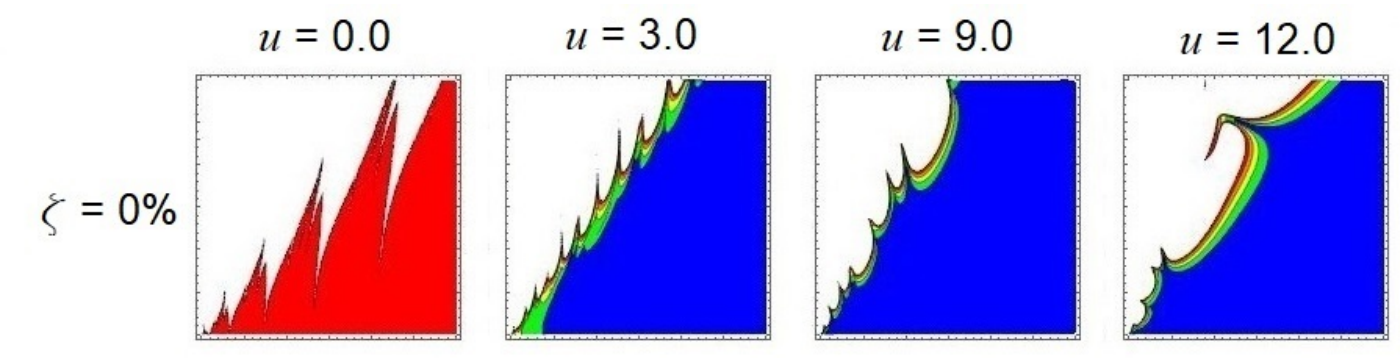

$|\lambda *|$
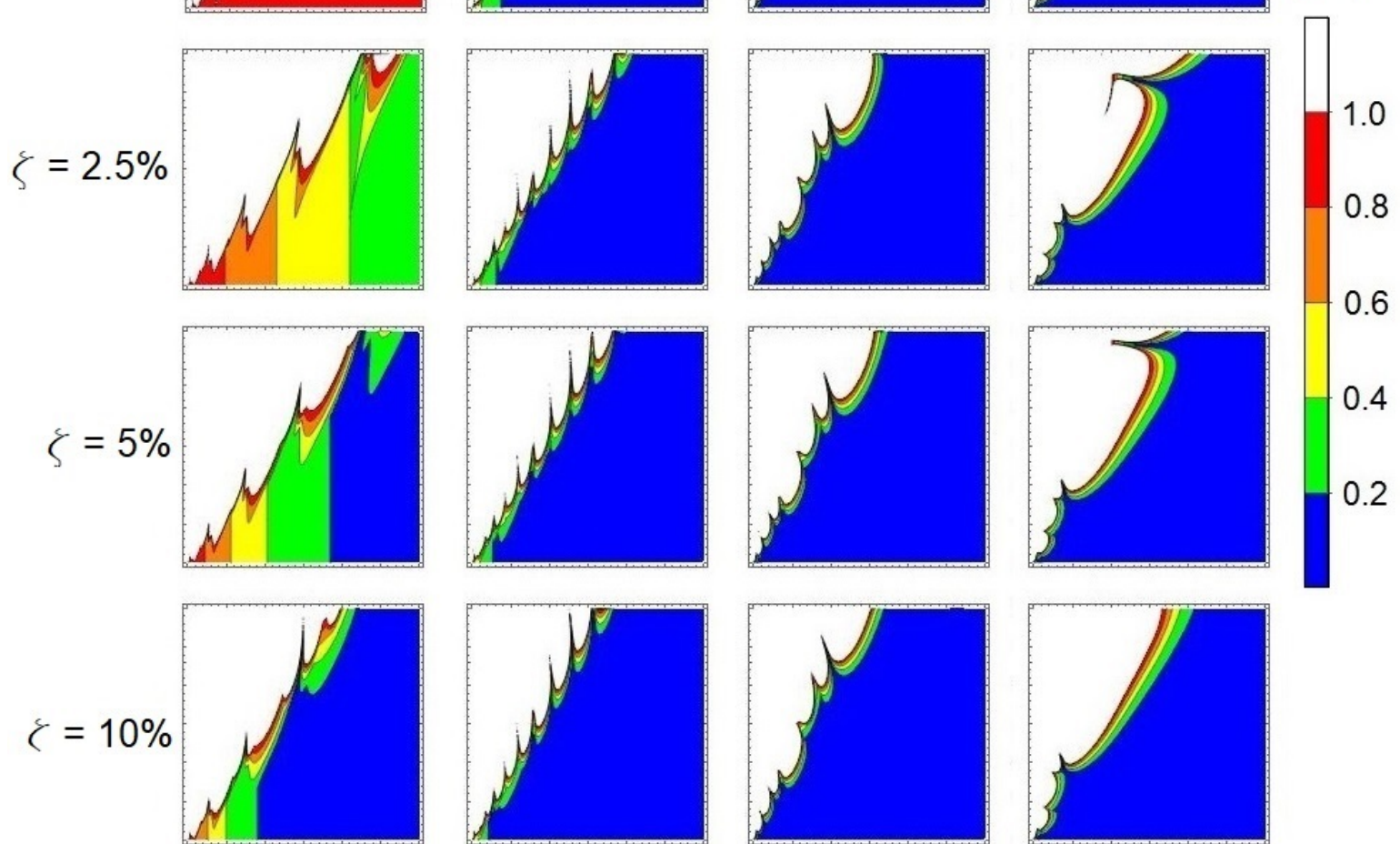

0.6
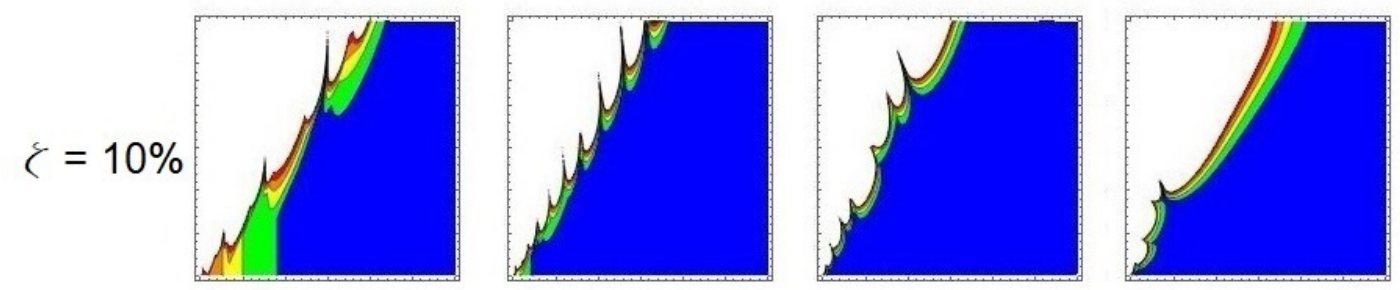
Observando o conjunto de diagramas apresentados nas Figuras 23, 24 e 25, é notável o aumento da região de soluções limitadas no plano de parâmetros de controle $(\delta ; \epsilon)$ quando ocorre o crescimento da velocidade de escoamento interno $u$ e da taxa de amortecimento estrutural $\zeta$. O aumento dessa região representa o crescimento nas condições das quais a solução trivial é estável.

O aumento de $u$ e $\zeta$ suaviza o encontro entre as franjas de estabilidade e o distancia do eixo das abcissas. Ocorre também o surgimento de diversas franjas menores ao longo de toda a fronteira (ver Figuras 23, 24 e 25 quando $u=3,0$ ). No entanto, na Figura 25 para $u=12,0$ ocorre a diminuição da quantidade de franjas e o alisamento da superfície da fronteira. Ainda observando a Figura 25 com $u=12,0$, antes do alisamento total da fronteira com o aumento de $\zeta$ é possível observar que o diagrama de estabilidade na posição $u=12,0$ e $\zeta=0,0 \%$ possui uma franja com formato incomum. Esta franja está localizada na parte superior do diagrama e apresenta inclinação em direção ao eixo das abcissas.

O caso apresentado na Figura 26 refere-se ao problema de instabilidade paramétrica para o problema clássico do pêndulo duplo. Analisando o diagrama de estabilidade apresentado na Figura 26a, é possível ver que $\left|\lambda^{*}\right|$ é sempre muito próximo de 1 (escala de cor vermelha). Com o aumento da taxa de amortecimento estrutural e da velocidade de escoamento interno, o valor de $\left|\lambda^{*}\right|$ passa a ser muito próximo de 0 (escala de cor azul). Ocorre uma variação abrupta na escala de cores nas regiões de fronteira como mostra a Figura 26d. É importante ressaltar que a variação de $\left|\lambda^{*}\right|$ não oferece informações sobre a estabilidade do sistema, uma vez que, de acordo com a Teoria de Floquet o sistema é estável quando $\left|\lambda^{*}\right| \leq 1,0$ e nada é dito sobre a variação do valor entre 0,0 e 1,0.

A solução da equação de Mathieu (Diagrama de Strutt) apresentada na Figura 10 possui franjas uniformes e que não apresentam erosão. Já o caso referente ao problema do pêndulo duplo, que respeita o mesmo conjunto de parâmetros do problema do pêndulo simples de massa concentrada, revela erosão nas franjas principais do diagrama fazendo com que apareçam sub-franjas ou franjas secundárias como é mostrado na Figura 26a. 
Figura 26 - Diagramas de estabilidade para o caso $\beta=0,750$

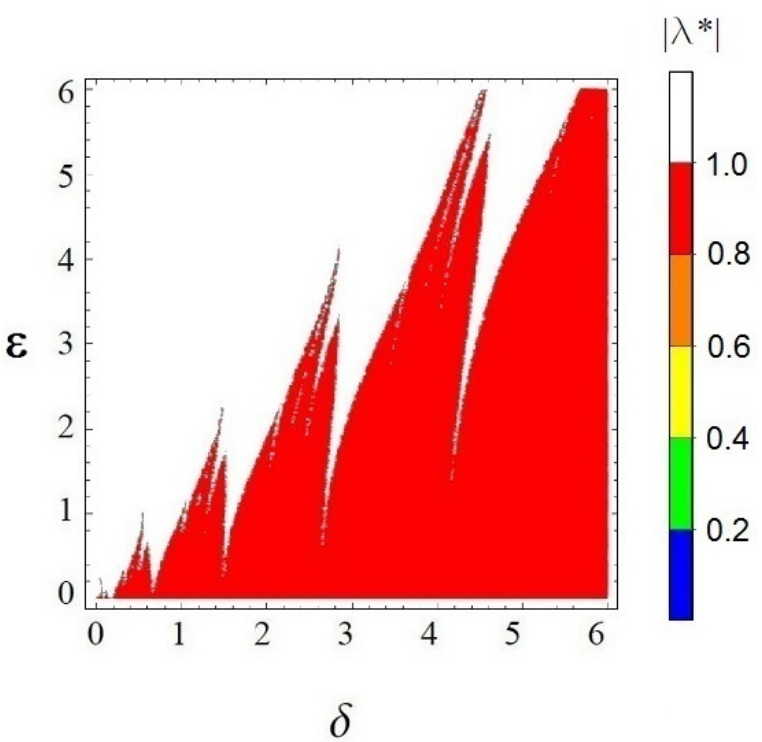

(a) $u=0,0 ; \zeta=0,0 \%$.

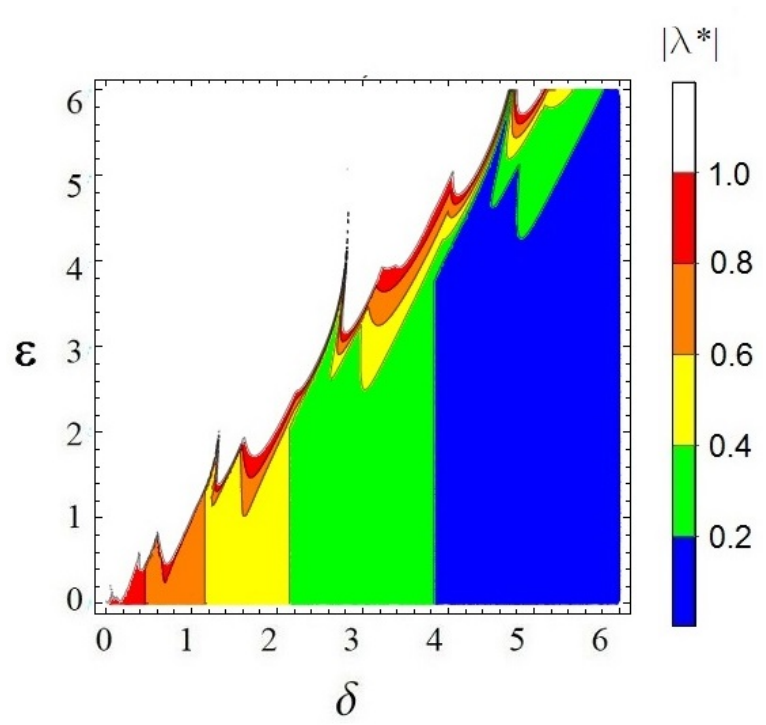

(c) $u=0,0 ; \zeta=5,0 \%$.

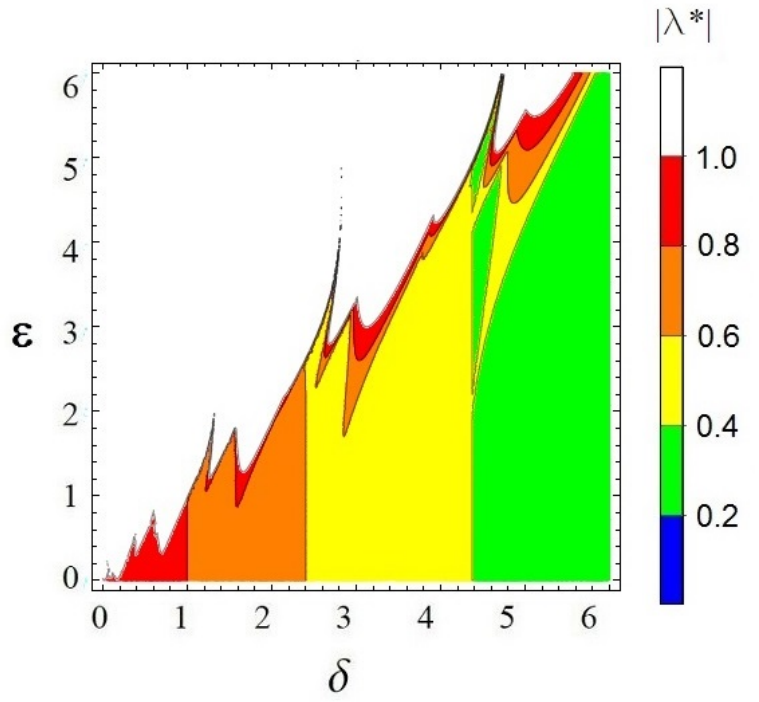

(b) $u=0,0 ; \zeta=2,5 \%$.

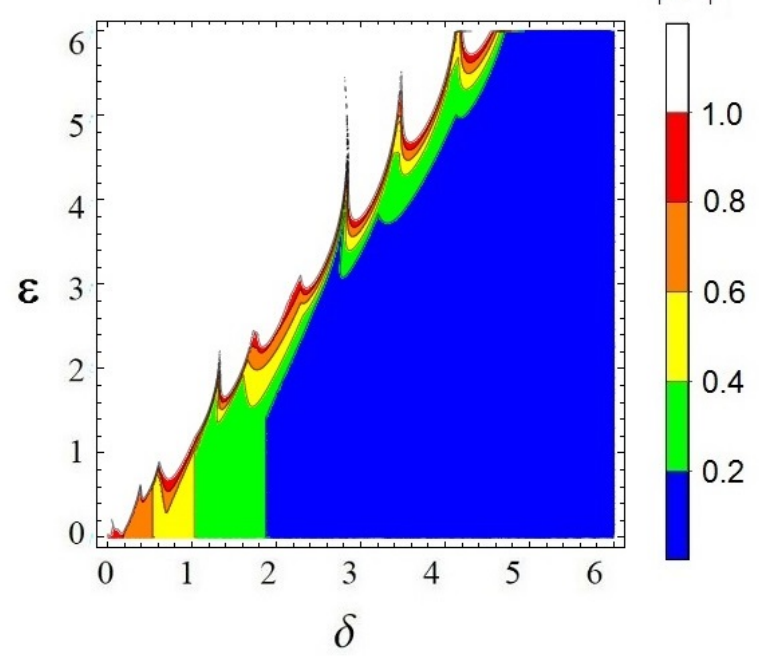

(d) $u=0,0 ; \zeta=10,0 \%$.

A Figura 27 foi retirada do conjunto de diagramas apresentados na Figura 25 para $\beta=0,750$. Quatro diagramas para o valor de velocidade de escoamento interno igual a $u=12,0$ e diferentes valores de amortecimento estrutural $\zeta$ são mostrados na Figura 27. A Figura 27a chama atenção por apresentar uma franja com formato incomum, apresentando inclinação em direção ao eixo das abscissas. Esta franja diminui de tamanho até desaparecer conforme o valor do amortecimento estrutural $\zeta$ aumenta. Isso reitera que o aumento do amortecimento no sistema tende a suavizar a região de fronteira entre as soluções limitadas e ilimitadas. 
Figura 27 - Diagramas de estabilidade para o caso $\beta=0,750$

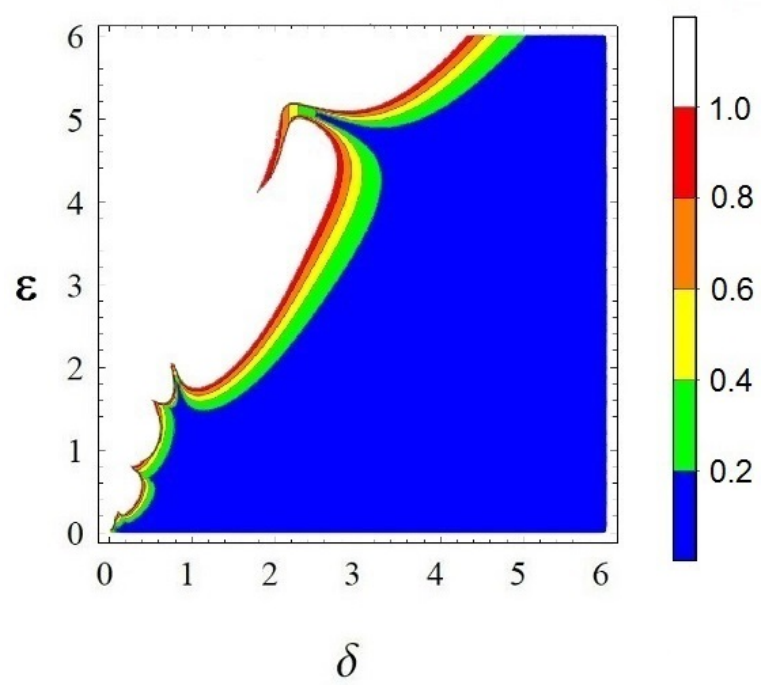

(a) $u=12,0 ; \zeta=0,0 \%$.

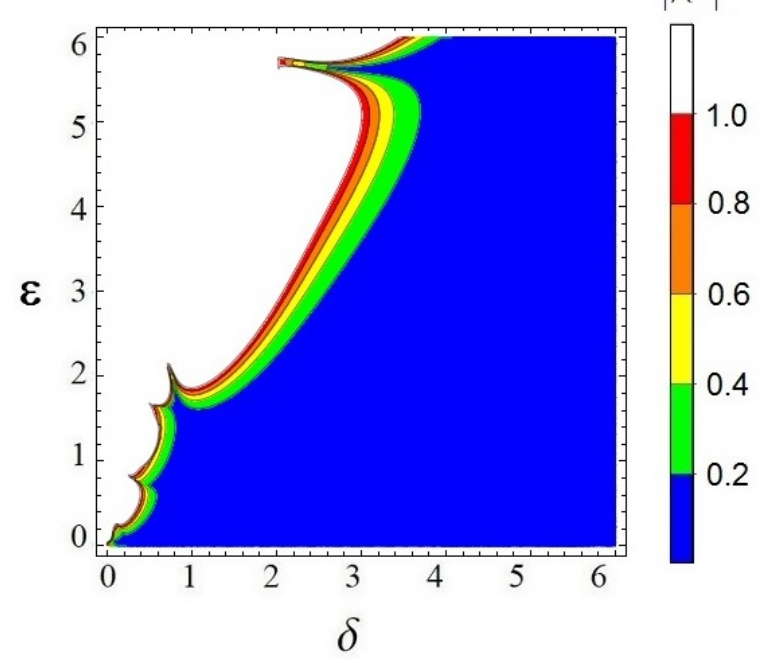

(c) $u=12,0 ; \zeta=5,0 \%$.

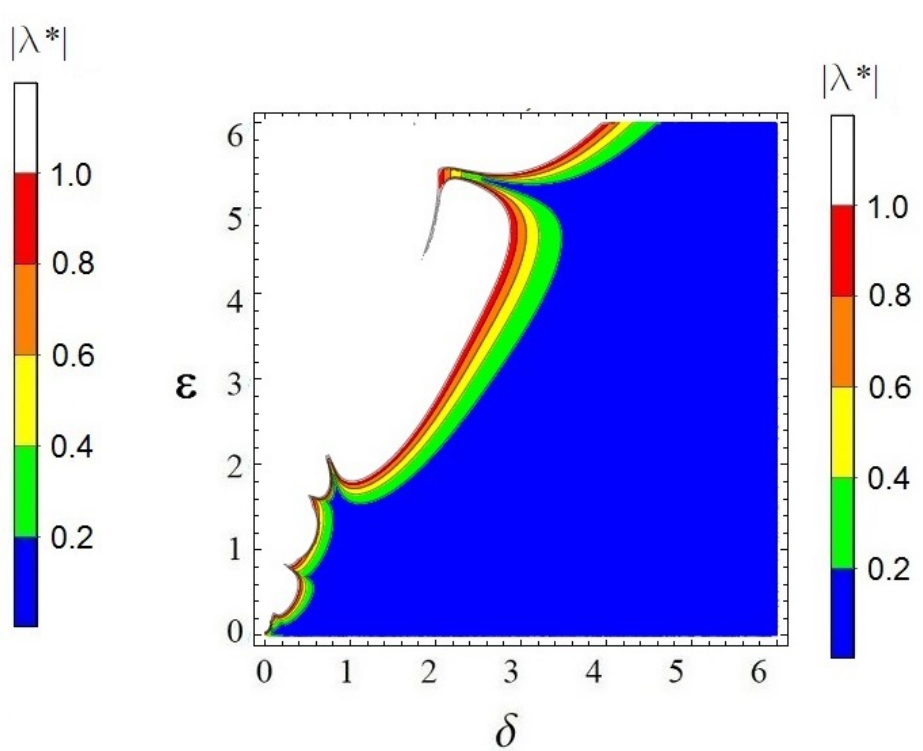

(b) $u=12,0 ; \zeta=2,5 \%$.

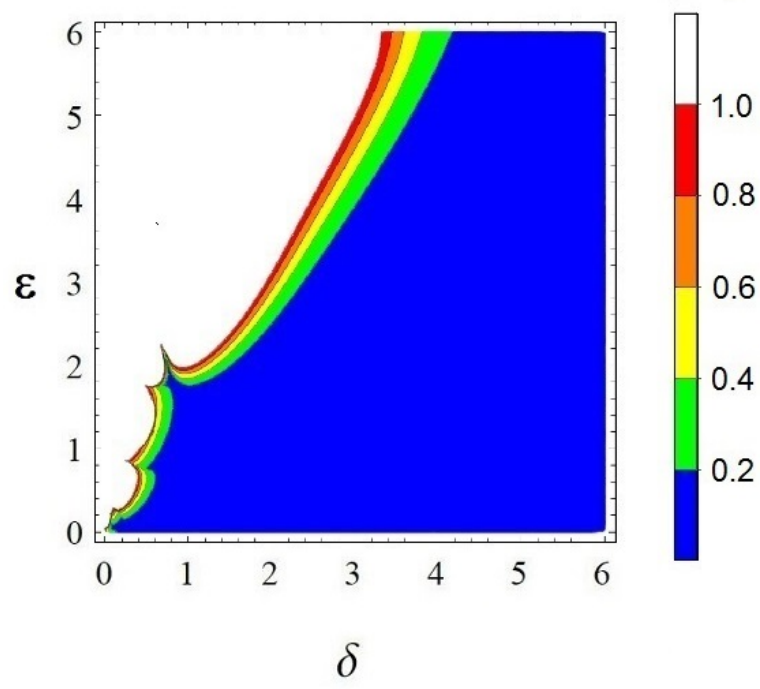

(d) $u=12,0 ; \zeta=10,0 \%$.

O leitor deve notar que um retorno ao caso com $\beta=0,500$ é agora feito. Observando os resultados apresentados na Figura 24 (verificar a diferença do padrão de respostas entre os diagramas para $u=9,0$ e $u=12,0)$, percebe-se que com o aumento da velocidade de escoamento interno $u=12,0$ duas franjas posicionadas no centro do diagrama, próximas a $(\delta=3,0 ; \epsilon=4,0)$, começam a se afastar da região que apresenta soluções limitadas. Desta forma, foram escolhidos alguns valores de velocidades $u$ superiores a 12,0. Logo, para $u=13,4$ foi observado o fenômeno apresentado na Figura 28. 
Figura 28 - Diagramas de estabilidade para o caso $\beta=0,500$

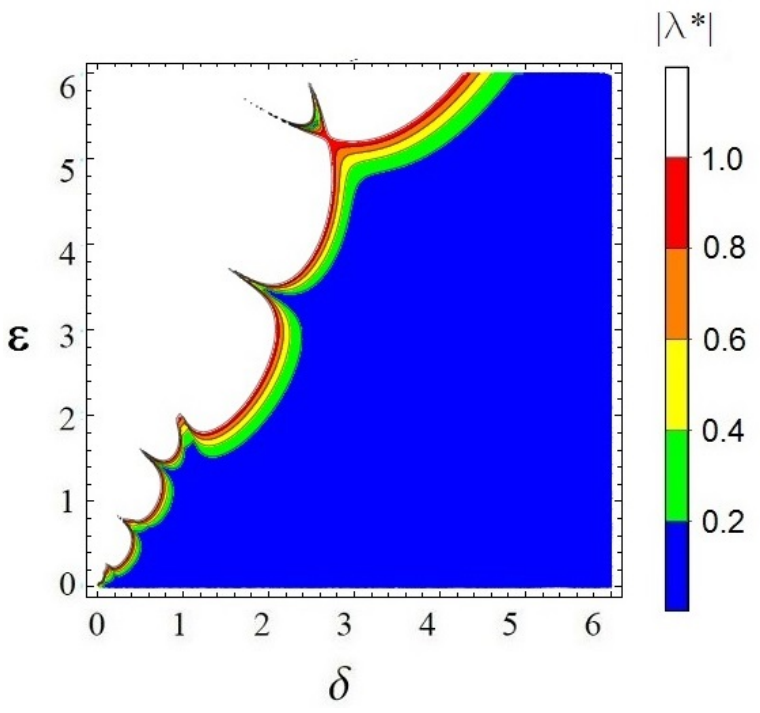

(a) $u=13,4 ; \zeta=0,0 \%$.

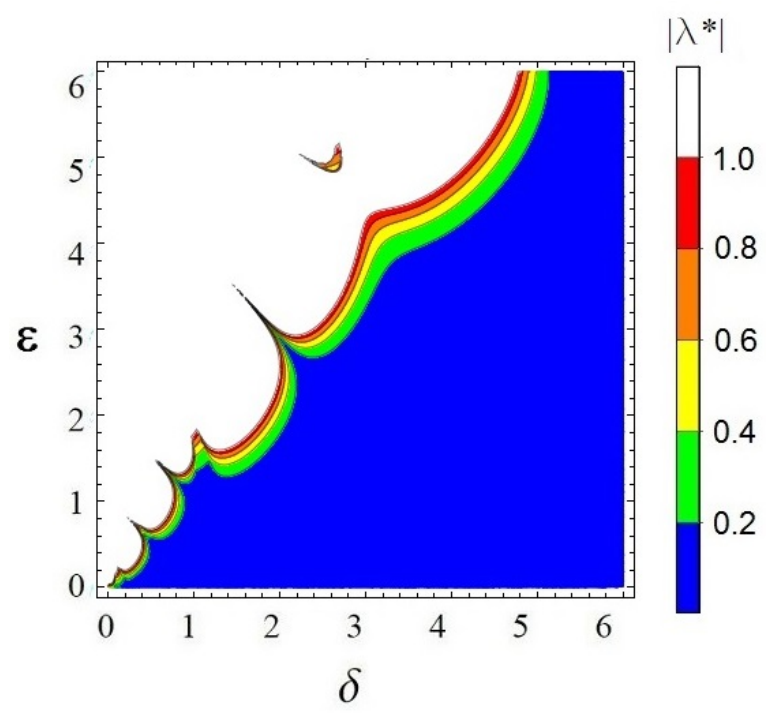

(c) $u=13,4 ; \zeta=5,0 \%$.

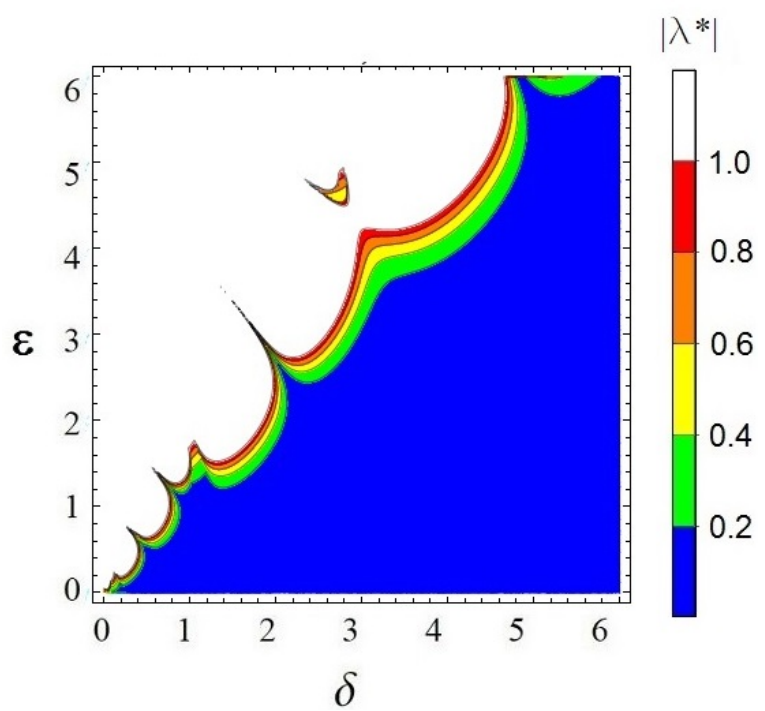

(b) $u=13,4 ; \zeta=2,5 \%$.

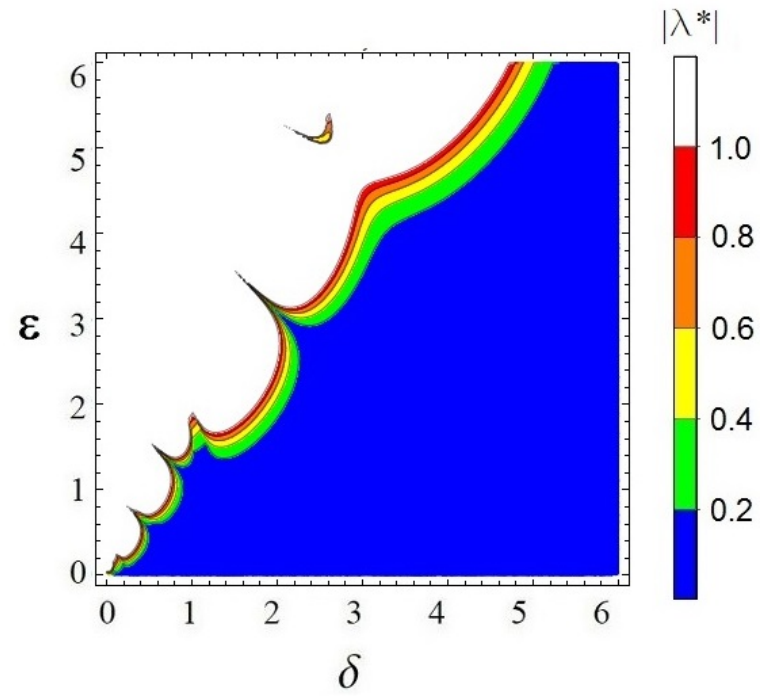

(d) $u=13,4 ; \zeta=10,0 \%$.

No conjunto de imagens apresentadas na Figura 28, é mostrada a situação na qual o par de franjas está na iminência de se desprender da região principal de estabilidade do sistema (ver Figura 28a). É então possível perceber que o aumento da taxa de amortecimento estrutural auxilia no processo de descolamento e posteriormente na redução até o desaparecimento da região secundária de estabilidade (ver Figuras 28b, 28c e 28d). Esta região secundária de estabilidade é definida aqui como sendo uma "ilha" de soluções limitadas cercada por soluções ilimitadas.

Esse fenômeno de aparecimento da "ilha" de estabilidade está associado ao grau de amortecimento do sistema, sendo possível afirmar que os parâmeros que controlam o amortecimento, $u$ e $\zeta$, estão presentes na matriz de amortecimento nas equações de movimento (ver, Equação 3.25). O parâmetro de velocidade $u$ também aparece na matriz 
de rigidez com sinal negativo, ou seja, influenciando na diminuição da rigidez do conjunto.

Foram escolhidos pares de parâmetros $(\delta ; \epsilon)$ de forma a verificar as séries temporais de pontos localizados dentro da "ilha" de estabilidade $(\delta=5,08 ; \epsilon=2,60)$ e fora, porém próximo a região da fronteira $(\delta=5,07 ; \epsilon=2,60)$. Por ser uma região de grande sensibilidade, uma pequena variação em um dos pares de parâmetros muda completamente o comportamento do sistema.

Observando a escala das amplitudes de variação dos ângulos apresentados é conclusivo que antes do sistema retornar a posição inicial ele oscila com grandes amplitudes tanto para $\theta_{1}$ quanto para $\theta_{2}$ (ver Figuras 29a e 29b). Nos casos que apresentaram respostas ilimitadas é visível que a amplitude de oscilação cresce ao longo do tempo, atingindo valores que não podem representar a viabilidade do sistema (ver Figuras 29c e 29d).

Figura 29 - Respostas das séries temporais dos ângulos $\theta_{1}$ e $\theta_{2}$ para $u=13,4 ; \zeta=5 \%$ e $\beta=0,500$

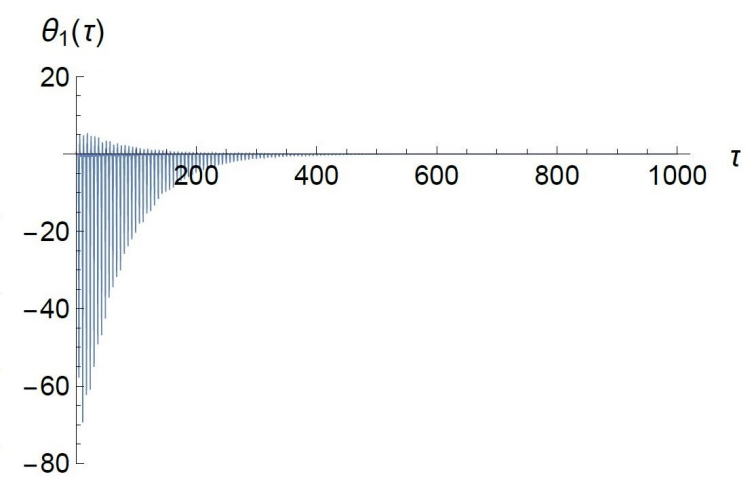

(a) $\delta=5,08 ; \epsilon=2,60$.

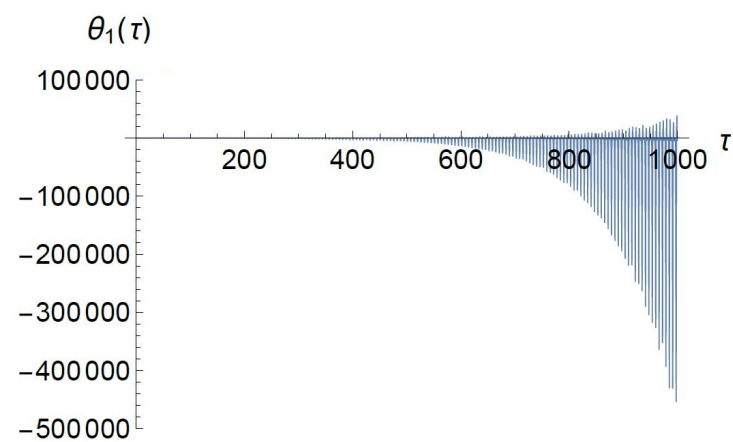

(c) $\delta=5,07 ; \epsilon=2,60$.

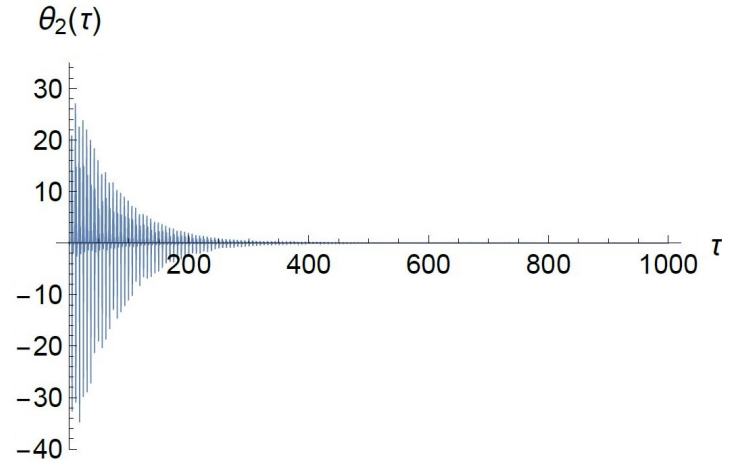

(b) $\delta=5,08 ; \epsilon=2,60$.

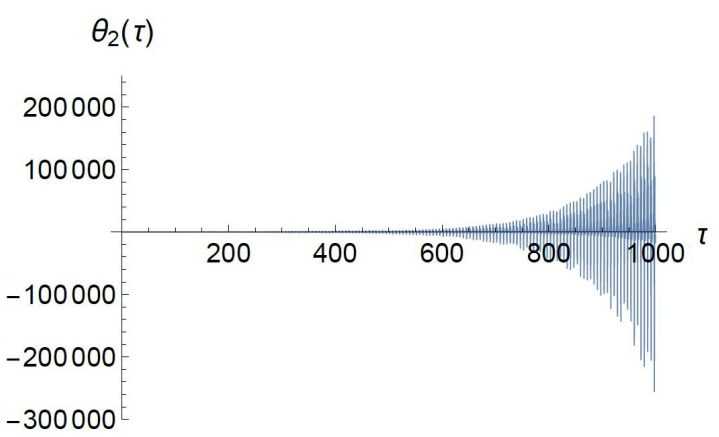

(d) $\delta=5,07 ; \epsilon=2,60$. 


\subsection{Análise Não Linear}

A análise não linear foi proposta para compreender melhor as descontinuidades apresentadas nas franjas principais quando $u=0,0$ e $\beta=0,75$ (ver Figura 26a), o surgimento da franja com inclinação em direção ao eixo das abscissas quando $u=12,0$ e $\beta=0,75$ (ver Figura 27a) e também o aparecimento da "ilha" de estabilidade quando $u=13,4$ e $\beta=0,50$ (ver Figura 28a). Além disso, a análise não linear permite ampliar o conhecimento sobre a dinâmica do sistema na região de respostas ilimitadas apresentadas nos diagramas obtidos a partir das análises lineares e também para velocidades supercríticas, que não podem ser estudadas com as equações linearizadas.

Para alguns casos, regiões específicas dos mapas de resposta pós-crítica serão colocadas em evidência para melhor visualização e explicação do comportamento. Os mapas irão apresentar a variação do movimento vertical da extremidade livre do conjunto de tubos $y_{t}(\tau)$ (ver Equação 4.1). Serão apresentados dois mapas, um contendo o valor da média de oscilação da série temporal $\bar{y}_{t}$, que representa o valor em torno do qual qual a resposta oscila. O outro irá mostrar os valores do desvio padrão $y_{t, s t d}$ para a série temporal de deslocamento da extremidade livre. Note que o desvio padrão permite quantificar a amplitude de oscilação da resposta.

Para a análise não linear foi utilizada a Equação 3.24, agora reapresentadas de forma a facilitar o entendimento por parte do leitor. Os mapas de resposta pós-crítica serão apresentados junto com os diagramas de estabilidade já apresentados na Seção 5.1. Isto será feito para facilitar a visualização e comparação dos resultados.

$$
\begin{aligned}
& \mathbf{M}^{*} \theta^{\prime \prime}+\mathbf{C}^{*} \theta^{\prime}+\mathbf{K}^{*} \theta=\mathbf{F}^{*} \\
& \mathbf{M}^{*}=\left[\begin{array}{cc}
\left(1-\lambda^{2}\right)\left(\frac{1+2 \lambda}{3}\right) & \frac{1}{2} \lambda^{2}(1-\lambda) \cos \left(\theta_{2}-\theta_{1}\right) \\
\frac{1}{2} \lambda^{2}(1-\lambda) \cos \left(\theta_{2}-\theta_{1}\right) & \frac{1}{3} \lambda^{3}
\end{array}\right] \\
& \mathbf{C}^{*}=\left[\begin{array}{cc}
u \psi(1-\lambda)^{2} \sqrt{\beta \delta} & u \psi \lambda(1-\lambda) \sqrt{\beta \delta} \cos \left(\theta_{2}-\theta_{1}\right) \\
u \psi \lambda(1-\lambda) \sqrt{\beta \delta} \cos \left(\theta_{2}-\theta_{1}\right) & u \psi \lambda^{2} \sqrt{\beta \delta}
\end{array}\right]+ \\
& +\left[\begin{array}{cc}
0 & u \psi \lambda(1-\lambda) \sqrt{\beta \delta} \cos \left(\theta_{2}-\theta_{1}\right) \\
-u \psi \lambda(1-\lambda) \sqrt{\beta \delta} \cos \left(\theta_{2}-\theta_{1}\right) & 0
\end{array}\right]+ \\
& +\left[\begin{array}{cc}
0 & -\frac{1}{2} \lambda^{2}(1-\lambda) \sin \left(\theta_{2}-\theta_{1}\right) \theta_{2}^{\prime} \\
\frac{1}{2} \lambda^{2}(1-\lambda) \sin \left(\theta_{2}-\theta_{1}\right) \theta_{1}^{\prime} & 0
\end{array}\right]+\left[\begin{array}{ll}
C_{1,1}^{s} & C_{1,2}^{s} \\
C_{2,1}^{s} & C_{2,2}^{s}
\end{array}\right] \\
& \mathbf{K}^{*}=\delta \psi^{2}\left[\begin{array}{cc}
K_{1}+K_{2} & -K_{2} \\
-K_{2} & K_{2}
\end{array}\right]
\end{aligned}
$$




$$
\mathbf{F}^{*}=\left\{\begin{array}{c}
(1-\lambda)\left(u^{2} \lambda^{2} \delta \sin \left(\theta_{2}-\theta_{1}\right)+\frac{(1+\lambda)}{2}(\delta+\epsilon \cos \tau) \sin \theta_{1}\right) \\
\frac{\lambda^{2}}{2}(\delta+\epsilon \cos \tau) \sin \theta_{2}
\end{array}\right\}
$$

Reitera-se que a análise não linear permite o estudo da amplitude e média das respostas no domínio $(\delta ; \epsilon)$ em regiões onde a análise linear não é capaz de obter respostas limitadas. Foram escolhidos quatro valores de velocidade de escoamento interno $u$ para realização das análises não lineares.

Dos quatro valores selecionados, três são menores que as velocidades críticas para os respectivos adimensionais de massa $\beta$ e uma é maior, porém muito próxima ao valor limite $\left(u=16,0\right.$, ligeiramente maior que $u_{c}=15,85$ para $\left.\beta=0,750\right)$. Os valores calculados das velocidades críticas para cada valor de $\beta$ podem ser vistos na Tabela 5 , já apresentada na Seção 5.1.

O primeiro caso analisado foi o problema do pêndulo duplo preenchido com fluido sem escoamento interno $u=0,0$, adimensional de massa $\beta=0,750$ e sem amortecimento estrutural $\zeta=0 \%$ (ver Figura 30). Nas Figuras 30b e 30c são apresentadas as variações de $y_{t, s t d}$ e $\bar{y}_{t}$, respectivamente. Os mapas $y_{t, s t d}$ e $\bar{y}_{t}$ revelam duas regiões que se destacam. A primeira delas preenchida pela cor roxa e que representa pequenas amplitudes de oscilação (associadas a baixos valores de $y_{t, s t d}$ ) em torno de valores médios $\bar{y}_{t}$ próximos a 0 .

A segunda região (a que não está preenchida pela cor roxa) apresenta uma mistura de respostas com diferentes escalas de cores impossibilitando identificar um padrão. Apesar da falta de padrão, a escala de cor possibilita identificar que o sistema chega a oscilar com amplitudes na ordem do comprimento de um dos segmentos de tubo. A mistura da escala de cor nos mapas de resposta pós-crítica de $y_{t, s t d}$ e $\bar{y}_{t}$ na região de regime pós-crítico é aqui identificada como sendo caracterizada por erosão no plano de parâmetros $(\delta ; \epsilon)$.

As respostas apresentadas na região caracterizada por pequenas amplitudes (preenchidas pela cor roxa) observadas nas Figuras 30b e 30c guardam certa aderência com a região de respostas limitadas para a análise linear observada na Figura 30a. Dessa forma, a análise não linear deve recuperar o formato das franjas da análise linear. As franjas e sub-franjas que aparecem na Figura 30a não apareceram com os mesmo nível de detalhe nas Figuras 30b e 30c. Apesar de não apareceram com mesmo nível de detalhe, é possível observar nas extremidades das franjas dos mapas $y_{t, s t d}$ e $\bar{y}_{t}$ o aparecimento de algumas descontinuidades que poderiam representar a formação dessas sub-franjas. 
Figura 30 - Mapa de amplitude e estatística da resposta não linear $y_{(\tau)} \cdot u=0,0, \zeta=0 \%$ e $\beta=0,750$.

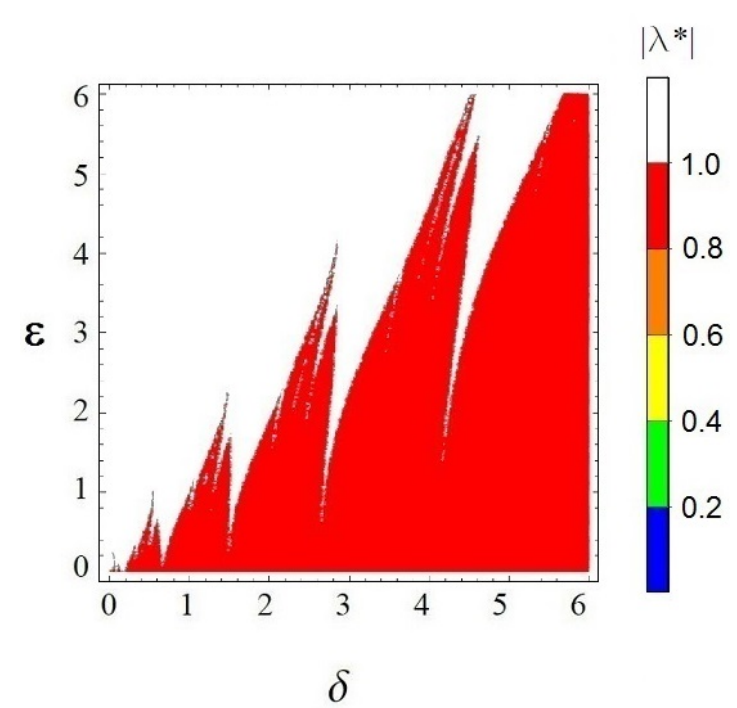

(a) Diagrama de estabilidade linear.

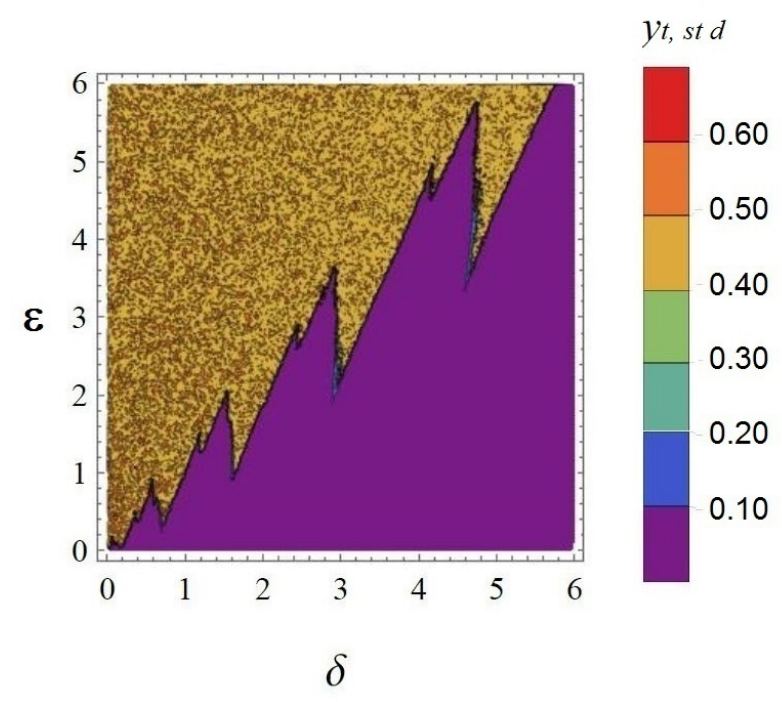

(b) $y_{t, s t d}(\delta ; \epsilon)$

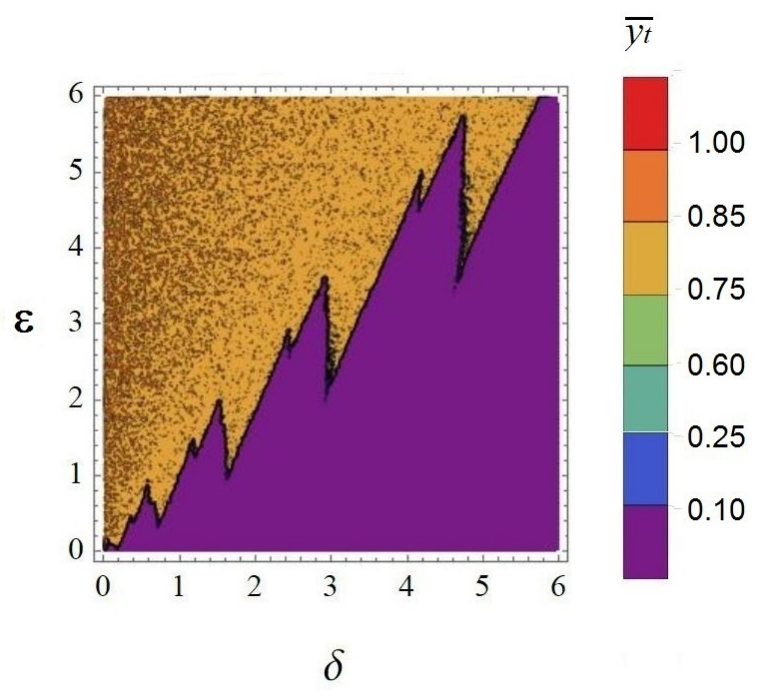

(c) $\bar{y}_{t}(\delta ; \epsilon)$

Nas Figuras 31 e 33 são estudados os casos com velocidade subcrítica $u=12,0$ e $\beta=0,750$ variando o valor da taxa de amortecimento estrutural adotado. Na Figura 31 é apresentado o caso onde foi considerado o sistema sem amortecimento estrutural. Na Figura 33 é apresentado o caso onde foi considerado o sistema com taxa de amortecimento estrutural $\zeta=10 \%$.

Na Figura 31a é reapresentado o diagrama de estabilidade para a análise linear e nas Figuras 31b e 31c são mostrados os mapas de resposta pós-crítica $\bar{y}_{t}$ e $y_{t, s t d}$. Os mapas de resposta pós-crítica também podem ser separados em duas regiões. A primeira região com pequenas amplitudes de movimento no qual o conjunto oscila em torna da posição de equilíbrio (região preenchida pelas cores roxa e azul escuro). Na segunda região 
(preenchida pelas demais cores) o conjunto oscila com grandes amplitudes e em torno de posições diferentes da configuração inicial.

Diferente do que foi observado nas Figuras 30b e 30c, a região com grandes amplitudes apresentada nas Figuras 31b e 31c mostra uma melhor organização, onde áreas de mesma escala de cor podem sem observadas. Mesmo com um padrão mais bem definido, ainda é possível identificar certa erosão no plano de parâmetros nas Figuras 31b e 31c, principalmente quando $0,0<\delta<2,0 \times 0,0<\epsilon<6,0$.

Figura 31 - Mapa de amplitude e estatística da resposta não linear $y_{(\tau)} \cdot u=12,0, \zeta=0 \%$ e $\beta=0,750$.

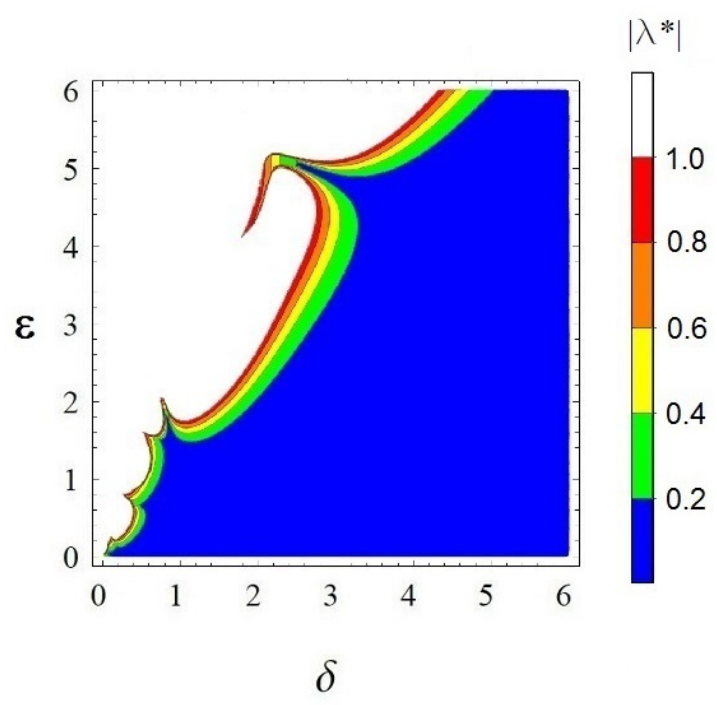

(a) Diagrama de estabilidade linear.

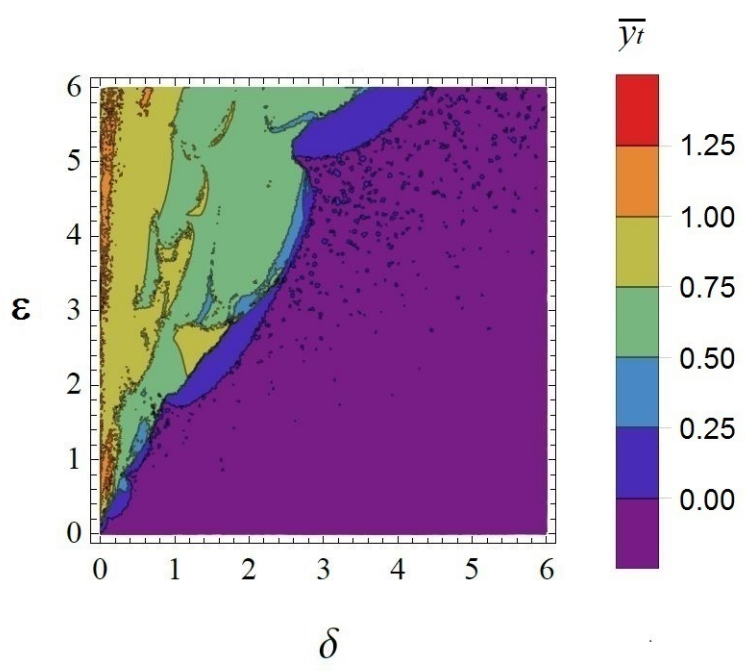

(b) $y_{t, s t d}(\delta ; \epsilon)$ $y t$, st d

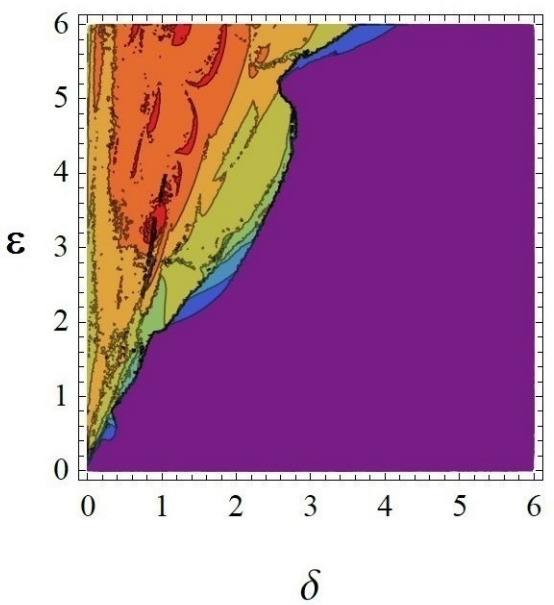

(c) $\bar{y}_{t}(\delta ; \epsilon)$

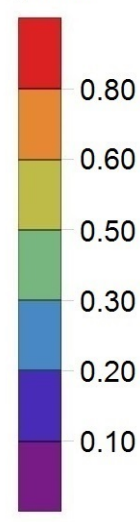

0.10

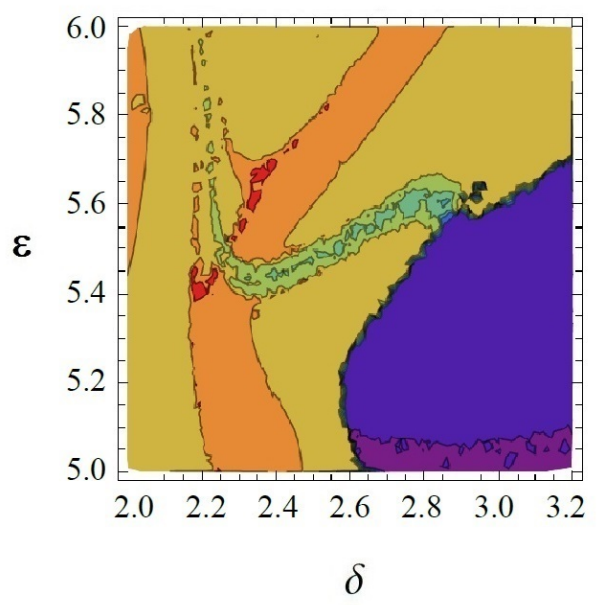

$y t$, st $d$

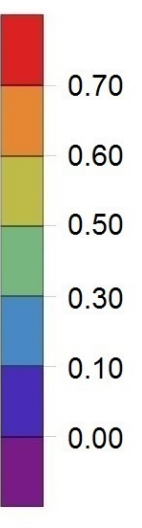

(d) $y_{t, s t d}(\delta ; \epsilon)$ - Região detalhada.

Na Figura 31a é mostrado que a franja localizada no intervalo $(2,0<\delta<4,0 \times$ $4,0<\epsilon<6,0)$ possui um formato diferente do usual. Observando o mesmo intervalo no 
plano de parâmetros para as respostas pós-críticas, é visto que um pequeno apêndice de formato semelhante aparece, mas com uma amplitude de escala ligeiramente maior que a representada pela cor roxa. A Figura 31d é uma ampliação da região do plano de parâmetros na área onde este apêndice foi observado para o caso de $\bar{y}_{t}$ apresentado na Figura 31c.

O apêndice apresentado na Figura 31d atravessa duas regiões com escalas de amplitude maiores (cores amarelo e laranja), além de apresentar os menores valores de escala em sua região central (azul claro). A fim de verificar a mudança de comportamento da série temporal em um ponto interno e outro externo da área do apêndice, foram geradas as séries temporais para $(\delta ; \epsilon)=(2,76 ; 5,60)$ apresentada na Figura 32a e $(\delta ; \epsilon)=(2,76 ; 5,75)$, exibida na Figura 32b.

Figura 32 - Respostas das séries temporais de $y_{t}(\tau)$ para $u=12,0, \zeta=0 \%, \beta=0,750 \mathrm{em}$ dois pontos no plano de parâmetros $(\delta ; \epsilon)$.

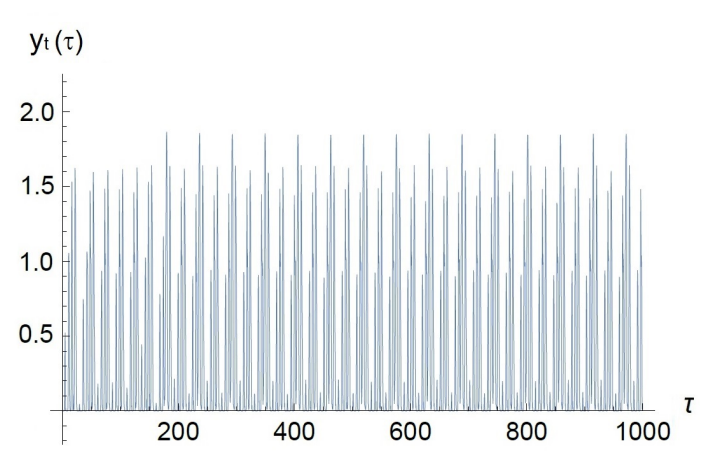

(a) $\delta=2,76 ; \epsilon=5,60$.

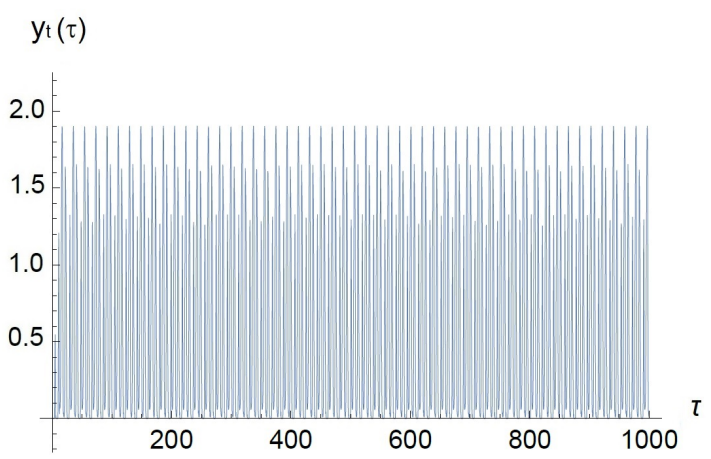

(b) $\delta=2,76 ; \epsilon=5,75$.

Nessas séries temporais são mostradas as diferenças de comportamento da variação da posição da extremidade livre do conjunto de tubos $y_{t}(\tau)$ para uma pequena mudança do parâmetro $\epsilon$. Comparando os valores numéricos das estatísticas aqui utilizadas, para o ponto localizados pelas coordenadas $(\delta ; \epsilon)=(2,76 ; 5,60)$ o valor da média é igual a 0,30 e o valor do desvio padrão é igual a 0,56 . Já para o ponto localizado das coordenadas $(\delta ; \epsilon)=(2,76 ; 5,75)$ o valor da média é igual a 0,50 e o valor do desvio padrão é igual a 0,60 .

Com o aumento do valor da taxa de amortecimento estrutural $\zeta$ de $0 \%$ para $10 \%$, a franja que podia ser observada na Figura 31a deixa de aparecer na Figura 33a. Esse comportamento indica que, apesar de ser intuitivo o fato do aumento do amortecimento aumentar a região de respostas com soluções limitadas, ocorre exatamente o contrário, revelando uma competição entre os parâmetros efeitos da velocidade de escoamento interno e da taxa de amortecimento estrutural.

Ainda relacionado ao incremento na taxa de amortecimento $\zeta$, os resultados apresentados nos mapas de resposta pós-crítica $\bar{y}_{t}$ e $y_{t, s t d}$ passam a apresentar uma variação brusca 
na escala de cores na região próxima à fronteira com a escala de cor roxa (ver Figuras 33b e 33c). Observando essa região de fronteira para a Figura 33b, percebe-se que ocorre a variação da escala de cor do azul escuro para o laranja no intervalo de $(2,5<\delta<3,5)$ e $(4,0<\epsilon<6,0)$. Para a Figura 33c, também acontece a mesma mudança brusca na escala de cores no mesmo intervalo do plano de parâmetros $(\delta ; \epsilon)$. Ainda observando as Figuras 33b e 33c longe da fronteira as respostas voltam a apresentar aspectos de erosão no plano de parâmetros quando $(0,0<\delta<1,7)$ e $(0,0<\epsilon<6,0)$.

Figura 33 - Mapa de amplitude e estatística da resposta não linear $y_{(\tau)} \cdot u=12,0, \zeta=10 \%$ e $\beta=0,750$.

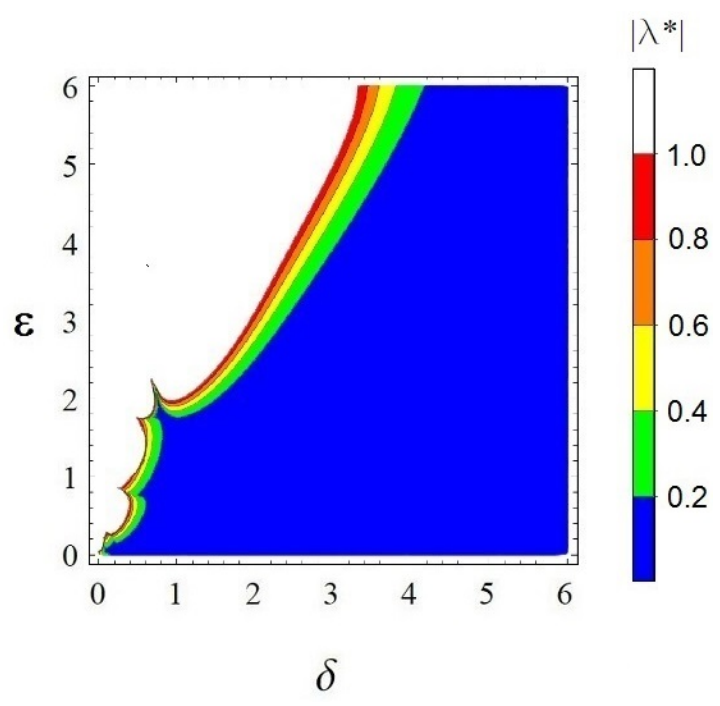

(a) Diagrama de estabilidade linear.

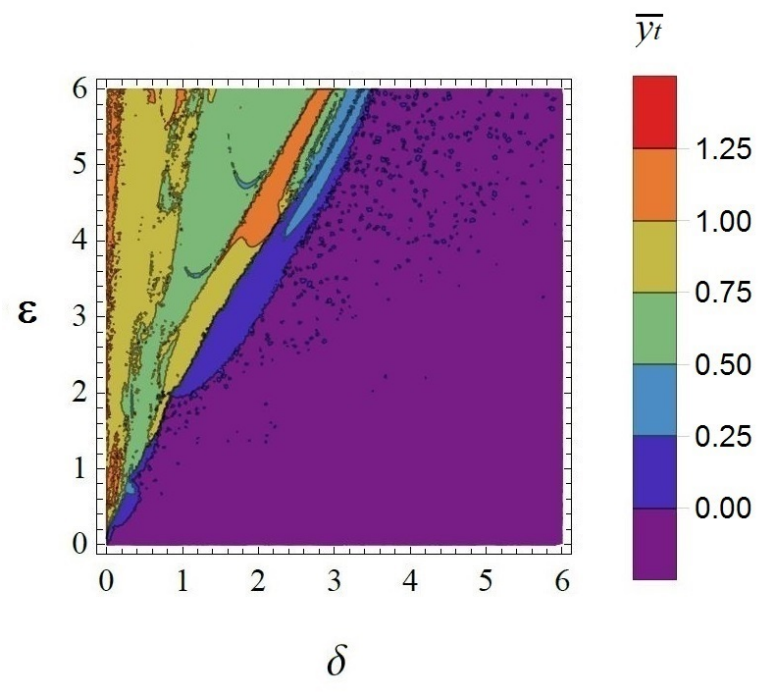

(b) $\bar{y}_{t}(\delta ; \epsilon)$

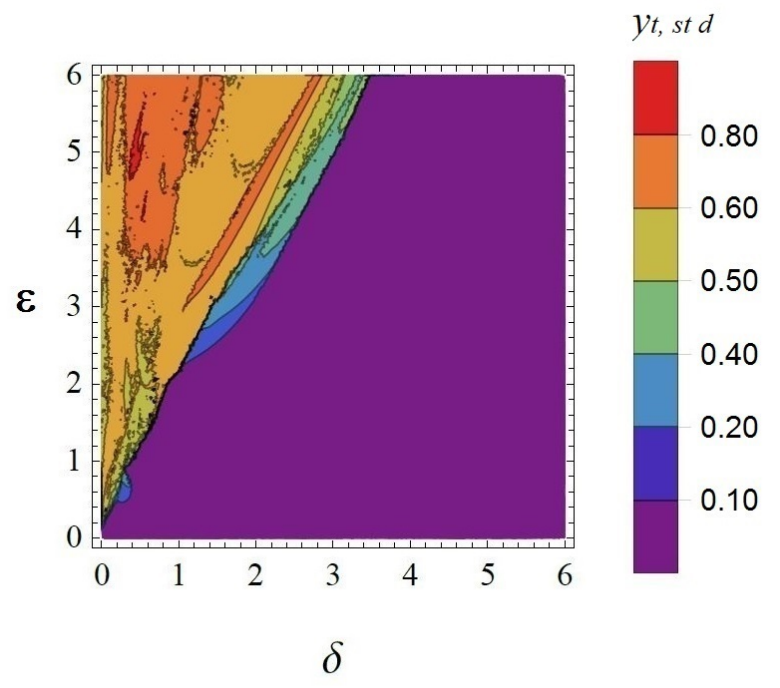

(c) $y_{t, s t d}(\delta ; \epsilon)$

Pode ser notado que ocorre uma pequena erosão no plano de parâmetros $(\delta ; \epsilon)$ na região caracterizada por oscilações com baixa amplitude (cor roxa) na Figura 33b quando 
$(3,0<\delta<6,0)$ e $(4,0<\epsilon<6,0)$, direcionando a atenção para esta região é notável que pequenas falhas de cor azul escuro começam a aparecer espalhadas dentro da região de cor roxa.

Na Figura 34 são apresentados os mapas de respostas em regime pós-crítico para o problema do pêndulo duplo com velocidade de escoamento interno $u=13,4$, taxa de amortecimento estrutural $\zeta=5 \%$ e adimensional de massa $\beta=0,500$. A Figura 34a está sendo apresentada novamente de forma a facilitar as comparações entre os mapas obtidos pelas equações não lineares e linearizadas. Nela foi observado o aparecimento de uma região de soluções limitadas separada da região principal.

A fim de verificar se esta "ilha" de soluções limitadas ainda apareceria na análise com o modelo matemático não linear e também como seria o comportamento do sistema próximos a fronteira foram criados os mapas apresentados nas Figuras 34b e 34c. De modo a facilitar a visualização do formato da "ilha" foi gerada uma imagem aproximando essa região, como pode ser observado na Figura 34d

Atentando-se para as respostas com baixas amplitudes de oscilação dos mapas de resposta pós-crítica nas Figuras 34b e 34c e as soluções limitadas do diagrama de estabilidade apresentado na Figura 34a, é visível a semelhança entre a região colorida da resposta linearizada e a região roxa das respostas do modelo não linear. Apenas as extremidades das franjas que não são tão acentuadas nas respostas não lineares comparadas com as franjas das respostas linearizadas; ver intervalo $(0,0<\delta<3,0)$ e $(0,0<\epsilon<3,0)$. Já a "ilha" apareceu na mesma região $((2,0<\delta<3,0 \times 4,9<\epsilon<5,5)$ e com formato muito semelhante nas duas análises.

Nas Figuras 34b e 34c existe uma forte erosão no plano de parâmetros $(\delta ; \epsilon)$ para as respostas pós-críticas, isso pode ser visto no intervalo $(0,0<\delta<1,0)$ e $(0,0<\epsilon<6,0)$. Quanto menores os valores do parâmetro $\delta$, maior o efeito de erosão no plano de parâmetros da região de regime pós-critico. Note que a região onde ocorre o aparecimento da "ilha" $(2,0<\delta<3,0)$ e $(4,9<\epsilon<5,5)$, mostrada na Figura 34d, apresenta soluções com pequenas amplitudes de oscilação para as equações não lineares (área preenchida pela cor roxa).

De forma a aprofundar o estudo das respostas obtidas com parâmetros $\epsilon$ e $\delta$, foram escolhidos dois pontos no plano de parâmetros, um deles situado no interior da "ilha" e outro próximo a fronteira, porém, na região que apresenta respostas com grandes amplitudes. Essas séries temporais podem ser observadas na Figura 35, onde a Figura 35a ilustra a resposta obtida com um par $(\delta ; \epsilon)=(2,55 ; 5,15)$ localizado no interior da "ilha".

Nos dois pontos do plano de parâmetros escolhidos foram aplicadas as mesmas condições iniciais de deslocamentos. Como a condição inicial de deslocamentos é da ordem de um grau, na série temporal do ponto localizado no interior da "ilha", $(\delta ; \epsilon)=(2,55 ; 5,15)$, 
Figura 34 - Mapa de amplitude e estatística da resposta não linear $y_{(\tau)} \cdot u=13,4, \zeta=5 \%$ e $\beta=0,500$.

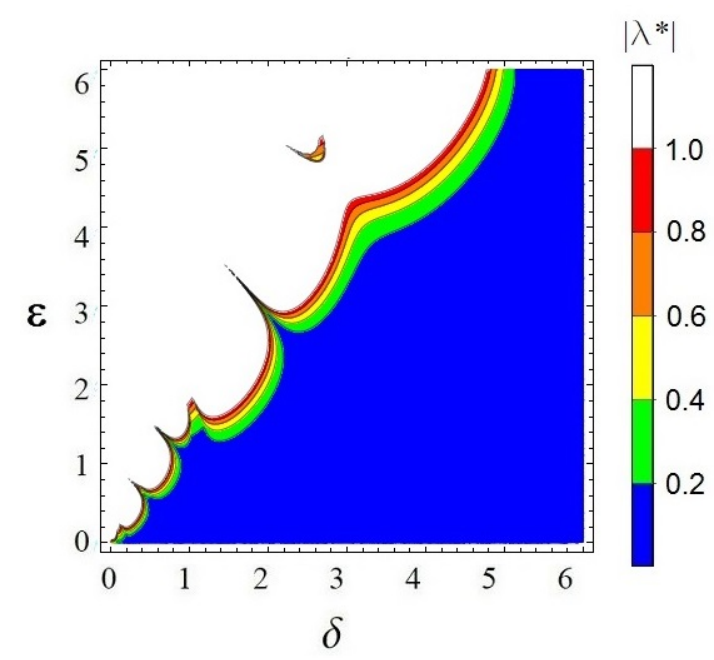

(a) Diagrama de estabilidade linear.

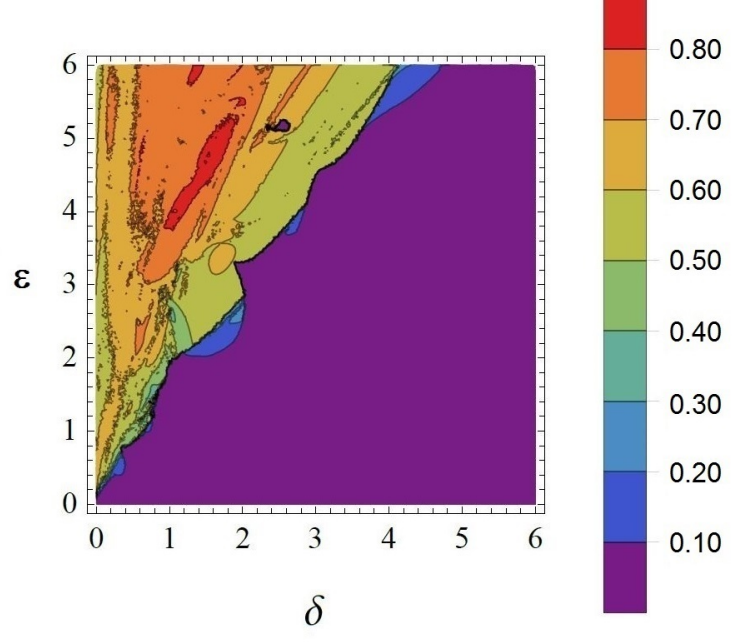

(b) $y_{t, s t d}(\delta ; \epsilon)$

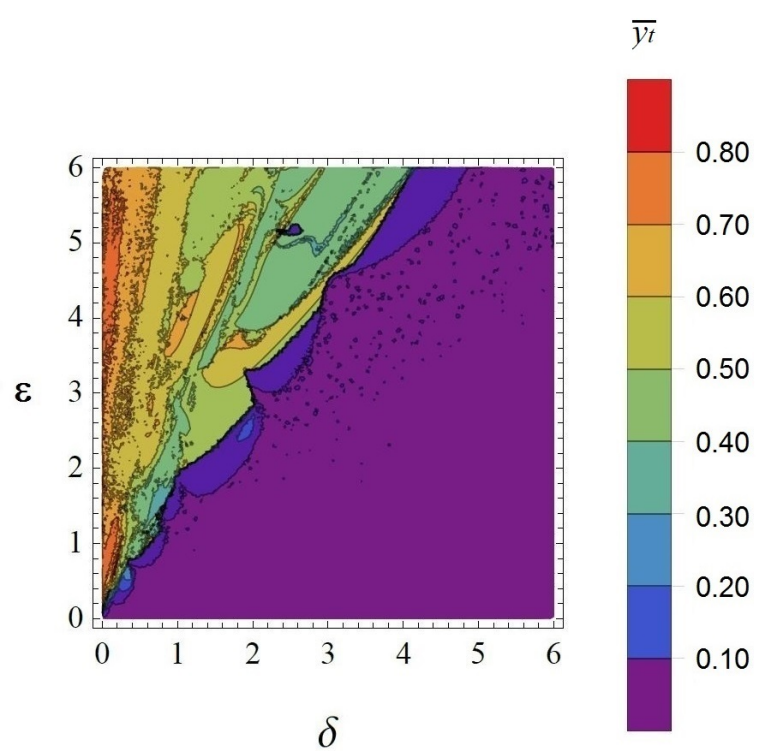

(c) $\bar{y}_{t}(\delta ; \epsilon)$

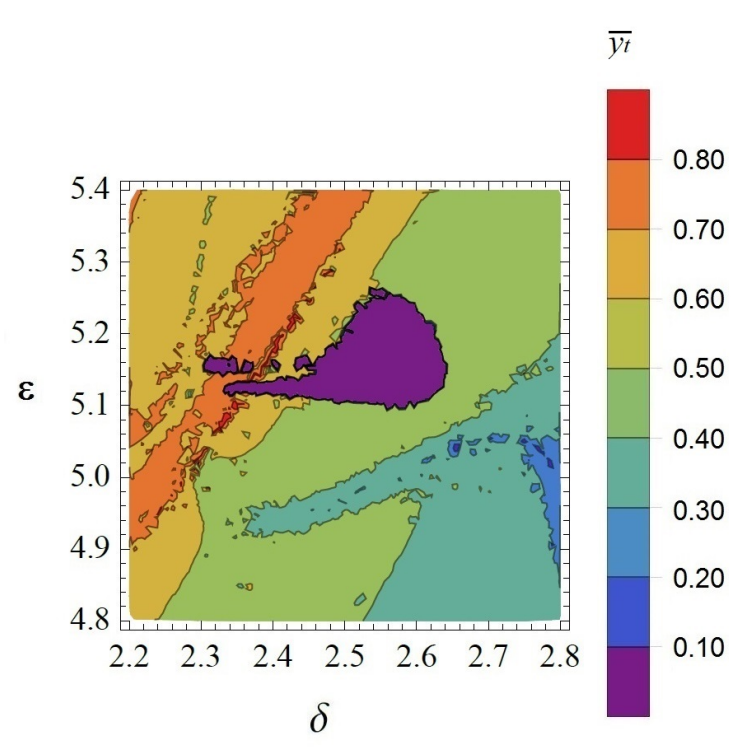

(d) $\bar{y}_{t}(\delta ; \epsilon)$ - Região detalhada.

ocorre um ligeiro aumento na amplitude de oscilação instantes antes de ser totalmente suprimida conforme é mostrado na Figura 35a. No ponto localizado fora da "ilha", $(\delta ; \epsilon)=$ $(2,50 ; 4,00)$, a amplitude das oscilações aumentam até atingir valores na ordem de uma vez e meia o comprimento total do conjunto de tubos (ver Figura 35b).

Em todas as séries temporais apresentadas neste trabalho em regiões de regime póscrítico, o sistema oscila com grandes amplitudes e sempre em torno de algum valor médio diferente da configuração trivial (ou seja, o conjunto de tubos na posição vertical). Isso pode ser observado nas séries temporais apresentadas e nos mapas de resposta pós-crítica 
Figura 35 - Respostas das séries temporais de $y_{t}(\tau)$ para $u=13,4, \zeta=5 \%, \beta=0,500 \mathrm{em}$ dois pontos no plano de parâmetros $(\delta ; \epsilon)$.

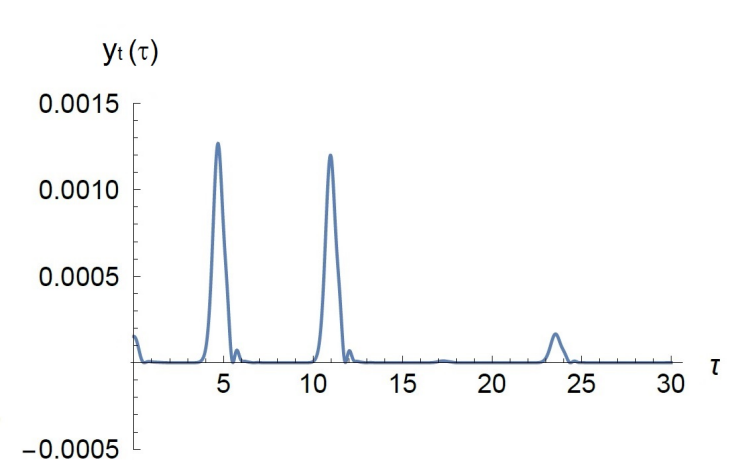

(a) $\delta=2,55 ; \epsilon=5,15$.

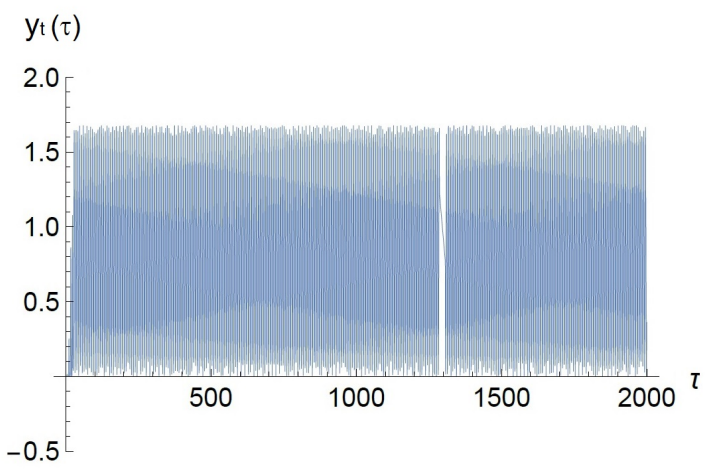

(b) $\delta=2,50 ; \epsilon=4,00$.

que representam o valor da média das séries temporais $y_{t}(\tau)$.

Até o momento foram discutidos apenas os resultados para os valores subcríticos da velocidade de escoamento interno $u$. Os resultados apresentados na Figura 36 são os mapas de amplitude para o conjunto de tubos com velocidade de escoamento interno $u=16,0, \zeta=0 \%$ e $\beta=0,750$. Esse caso é o único com velocidade de escoamento acima do super-crítico como pode ser visto na Tabela 5 .

Figura 36 - Mapa de amplitude e estatística da resposta não linear $y_{(\tau)} \cdot u=16,0, \zeta=0 \%$ e $\beta=0,750$.

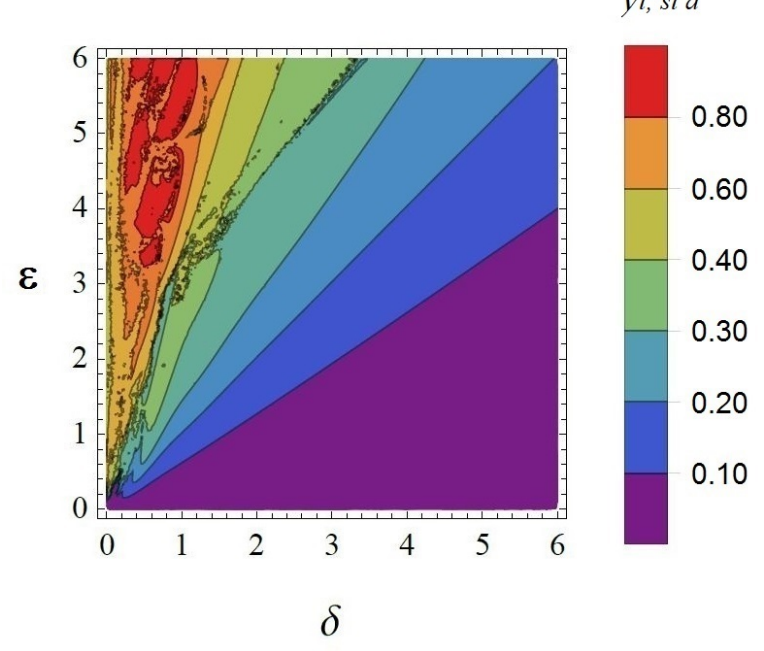

(a) $y_{t, s t d}(\delta ; \epsilon)$

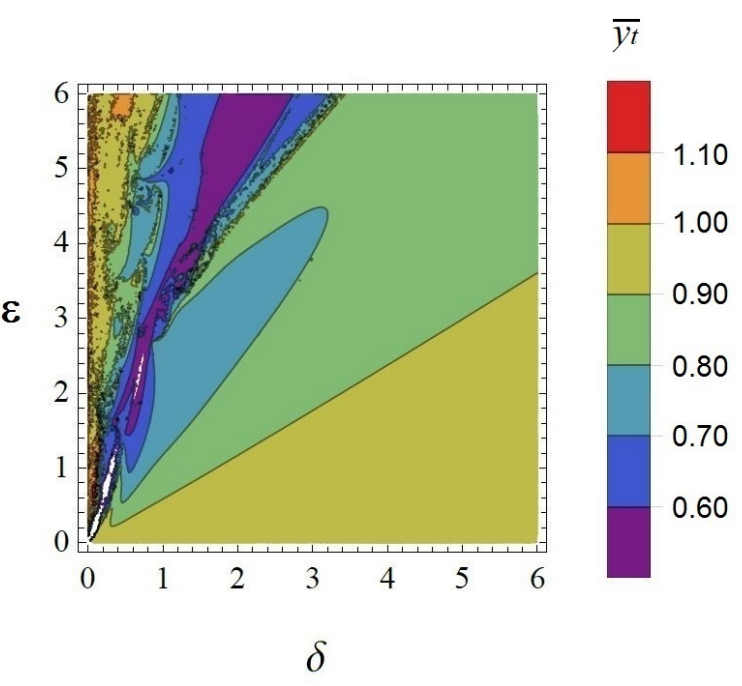

(b) $\bar{y}_{t}(\delta ; \epsilon)$

Como é mostrado na matriz de figuras para o caso com $\beta=0,750$ apresentado na Figura 25, conforme o valor da velocidade de escoamento interno aumenta no intervalo de $(0,0<u<1,0)$ as franjas dos diagramas diminuem de tamanho até que a região de fronteira entre as soluções limitadas e ilimitadas dos diagramas de estabilidade se tornem 
linhas regulares. Para o valor de velocidade de escoamento interno super crítica adotado $u=16,0$ as fronteiras entre as diferentes escalas de cor não apresentam mais franjas.

Anteriormente para valores de $(\delta ; \epsilon)$ nos quais o sistema costumava oscilar em torno da posição vertical (área preenchida pela cor roxa nas Figuras 30c, 31c, 33b e 34b) quando $u<u_{\text {crit }}$. Agora, para esses mesmos valores $(\delta ; \epsilon)$, o conjunto oscila em torno de uma nova posição média como poder ser visto pela região preenchida pela cor amarela na Figura 36b). Isso mostra que para velocidades de escoamento interno maiores que a velocidade crítica o sistema passa a ter um comportamento diferente, onde, para baixas amplitudes e frequências de movimento imposto no suporte o conjunto de tubo oscila em torno de uma posição diferente da solução trivial (conjunto de tubos posicionados na vertical).

Dessa forma, os valores de $u>u_{\text {crit }}$ são responsáveis por essa mudança do valor médio em torno do qual o sistema oscila em torno. Observando a escala para o valor médio das oscilações de $y_{t}(\tau)$ percebe-se que a extremidade livre do conjunto de tubos oscila próximo de 1,0 (cor amarela). Isso significa que $y_{t}(\tau)$ oscila próximo à posição horizontal.

É mostrado que, dependendo do par de amplitude e frequência do movimento imposto, o conjunto de tubos passa a se comportar de maneiras distintas. Na Figura 37 foram apresentadas as séries temporais para diversos pontos no plano de parâmetros $(\delta ; \epsilon)$. Foram escolhidos pontos considerando valores de $\delta$ próximos ao fim da escala do eixo das abscissas e valores de $\delta$ próximos ao início. 
Figura 37 - Respostas das séries temporais de $y_{t}(\tau)$ para $u=16,0, \zeta=0 \%, \beta=0,750 \mathrm{em}$ quatro pontos no plano de parâmetros parameters $(\delta ; \epsilon)$.

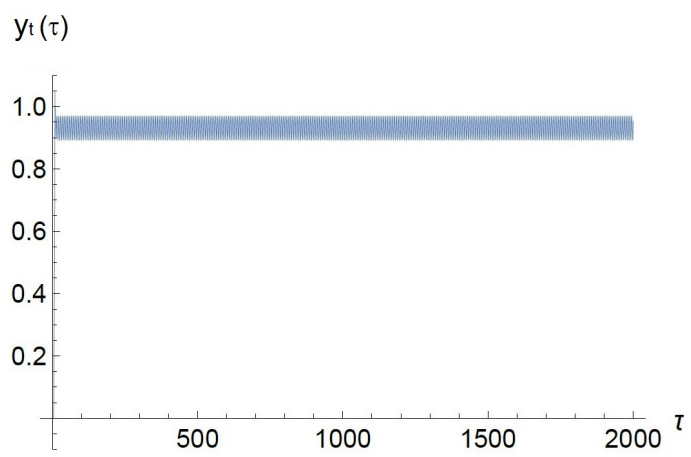

(a) $\delta=4,00 ; \epsilon=1,00$.

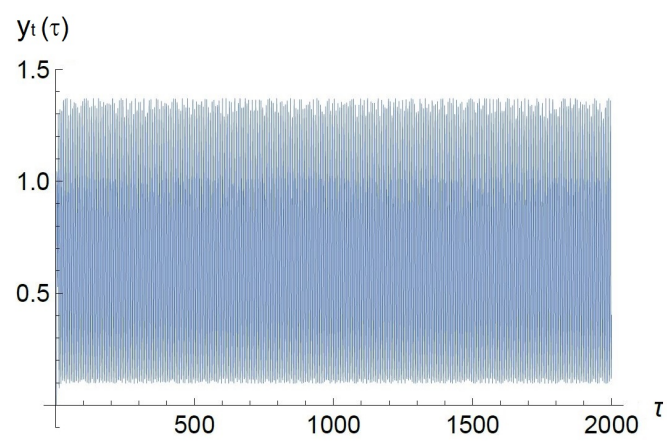

(c) $\delta=0,80 ; \epsilon=2,00$.

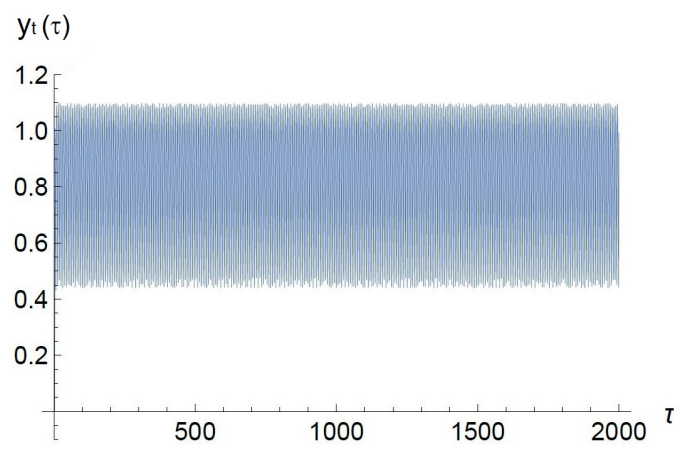

(b) $\delta=5,00 ; \epsilon=5,50$.

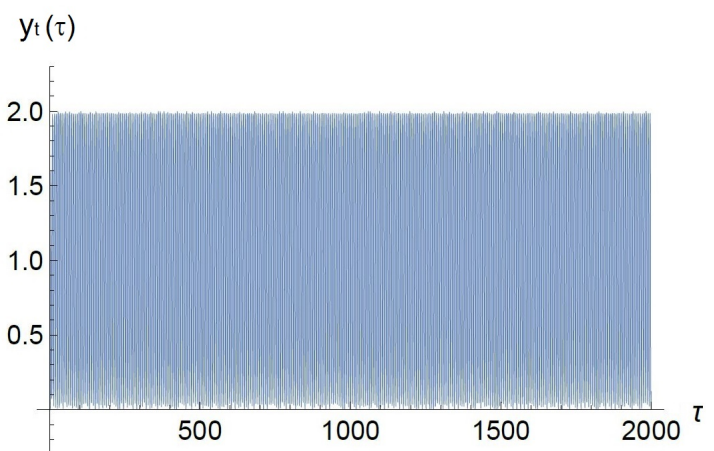

(d) $\delta=0,50 ; \epsilon=5,90$.

As Figuras 37a e 37b onde os valores de $\delta$ são maiores com relação aos outros pontos escolhidos ( $\delta=4,0$ e $\delta=5,0$, respectivamente), as amplitudes de oscilação da extremidade livre do tubo são na ordem de 0,2 e 0,6 , porém o valor médio em torno do qual o sistema oscila é na ordem de 0,9 e 0,78. Já para os casos apresentados nas Figuras 37c e $37 \mathrm{~d}$ onde os valores de $\delta$ são menores ( $\delta=0,8$ e $\delta=0,5$, respectivamente), as amplitudes de oscilação da extremidade livre do tubo são na ordem de 1,2 e 2,0 e o valor médio em torno do qual o sistema oscila é na ordem de 0,6 e 1,0. 


\section{Conclusão}

O objetivo deste trabalho foi realizar o estudo numérico da dinâmica de um conjunto de dois tubos rígidos e articulados entre si, submetidos a efeitos combinados de vibração induzida pelo escoamento interno e excitação paramétrica. Foi utilizada a Teoria de Floquet para a obtenção dos diagramas de estabilidade para o problema linearizado e foram criados mapas de respostas pós-críticas via integração numérica para o modelo não linear.

A variação dos valores do adimensional de massa $\beta$ simula a utilização de tubulação confeccionadas com diferentes materiais de variadas massas específicas. Esse efeito se mostra presente com a fragmentação de algumas regiões de estabilidade nos diagramas apresentados. Nas situações com maiores valores de $\beta$, essas fragmentações apresentam um aumento de tamanho. Esse efeito do aumento dos valores de $\beta$ ocasionam o surgimento de sub-franjas ou franjas secundárias no problema do pêndulo duplos sem escoamento interno.

A Teoria de Floquet se mostrou uma ferramenta bastante interessante, uma vez que ela permitiu percorrer uma vasta combinação de parâmetros em pouco tempo. Em poucos minutos foram verificadas as condições de estabilidade para milhares de pontos. Muito mais vantajoso do que verificar cada par de parâmetros individualmente por meio de simulações numéricas não lineares e que necessitam de um tempo de simulação muito longo.

A análise não linear mostrou a presença de erosão no plano de parâmetros que governa a excitação paramétrica. Essa erosão indica que o sistema é extremamente sensível à variação da frequência e amplitude da excitação paramétrica, especialmente nas regiões com grande amplitude do movimento imposto no topo do conjunto de tubos.

A análise não linear foi realizada para auxiliar no entendimento do comportamento nas regiões onde a análise linear não é capaz de apresentar qualquer tipo de resultado em regime sub-crítico (velocidades de escoamento interno abaixo daquela crítica) e para todo o regime super-crítico. Para os valores sub-críticos e na região de soluções ilimitadas, os mapas de amplitude mostraram que a media e o desvio padrão das oscilações da extremidade livre do conjunto de tubos $y_{t}(\tau)$ variam de acordo com o par de parâmetros escolhidos $(\delta ; \epsilon)$. Para algumas regiões, os mapas apresentaram marcas de erosão, um indicativo de mudanças bruscas nas respostas para pequenas variações dos valores dos parâmetros no modelo matemático.

Para velocidades de escoamento interno supercríticas, os mapas mostram que o conjunto de tubos oscila em torno de uma nova configuração média diferente da posição de equilíbrio para os casos subcríticos. Foi mostrado na análise linear e confirmado nos 
estudos com o modelo não linear que para valores específicos de velocidade de escoamento interno, taxa de amortecimento estrutural e adimensional de massa, uma área isolada de soluções limitadas surge dentro da região de soluções ilimitadas.

Fica a sugestão para trabalhos futuros incluir a investigação experimental do problema em tela. Os resultados experimentais poderão ser comparados com os resultados numéricos já apresentados. Também é interessante estudar os efeitos que as variações das condições iniciais do modelo matemático não linear apresentam na resposta. Finalmente, lembra-se que o problema aqui estudado é um modelo de ordem reduzida com diversas aplicações tecnológicas dentro do campo da engenharia. Desta forma, a metodologia aqui desenvolvida pode ser utilizada em modelos de maior ordem hierárquica. 


\section{Referências}

BENDER, C. M.; ORSZAG, S. A. Advanced Mathematical Methods for Scientists and Engineers. [S.1.]: McGraw-Hill, 1978.

BENJAMIN, T. B. Dynamics of a system of articulated pipes conveying fluid. i. theory. Proceedings of the Royal Society A: Mathematical, Physical and Engineering Sciences, The Royal Society, v. 261, n. 1307, p. 457-486, may 1961.

BENJAMIN, T. B. Dynamics of a system of articulated pipes conveying fluid. II. experiments. Proceedings of the Royal Society A: Mathematical, Physical and Engineering Sciences, v. 261, n. 1307, p. 487-499, may 1961.

BLEVINS, R. D. Formulas for natural frequency and mode shape. [S.l.]: Krieger, 1984.

BLEVINS, R. D. Flow-Induced Vibration. [S.1.]: Krieger, 2001.

BOHN, M. P.; HERRMANN, G. The dynamic behavior of articulated pipes conveying fluid with periodic flow rate. Journal of Applied Mechanics, ASME International, v. 41, n. 1, p. 55-62, mar 1974.

CASETTA, L.; PESCE, C. P. The generalized hamilton's principle for a non-material volume. Acta Mechanica, v. 224, n. 4, p. 919-924, jan 2013.

CHANDRASEKARAN, S.; CHANDAK, N.; ANUPAM, G. Stability analysis of TLP tethers. Ocean Engineering, Elsevier BV, v. 33, n. 3-4, p. 471-482, mar 2006.

CHATJIGEORGIOU, I.; MAVRAKOS, S. Bounded and unbounded coupled transverse response of parametrically excited vertical marine risers and tensioned cable legs for marine applications. Applied Ocean Research, Elsevier BV, v. 24, n. 6, p. 341-354, dec 2002 .

CHATJIGEORGIOU, I. K. Solution of the boundary layer problems for calculating the natural modes of riser-type slender structures. Journal of Offshore Mechanics and Arctic Engineering, ASME International, v. 130, n. 1, dec 2007.

FRANZINI, G. R. Tópicos de pesquisa em problemas de excitação paramétrica e de vibrações induzidas pelo escoamento. Livre docência, 2019.

FRANZINI, G. R.; MAZZILLI, C. E. N. Non-linear reduced-order model for parametric excitation of vertical and immersed slender rod. International Journal of Non-linear Mechanics, v. 80, p. 29-39, 2016.

FRANZINI, G. R.; PESCE, C. P.; SALlES, R.; GONÇALVES, R. T.; FUJARRA, A. L. C.; MENDES, P. Experimental analysis of a vertical and flexible cylinder in water: Response to top motion excitation and parametric resonance. Journal of Vibration and Acoustics, ASME International, v. 137, n. 3, jun 2015.

GREGORY, R. W.; PAÏDOUSSIS, M. P. Unstable oscillation of tubular cantilevers conveying fluid. i. theory. Proceedings of the Royal Society A: Mathematical, Physical and Engineering Sciences, v. 293, n. 1435, p. 512-527, aug 1966. 
GREGORY, R. W.; PAÏDOUSSIS, M. P. Unstable oscillation of tubular cantilevers conveying fluid. II. experiments. Proceedings of the Royal Society A: Mathematical, Physical and Engineering Sciences, v. 293, n. 1435, p. 528-542, aug 1966.

HOUSNER, G. W. Bending vibrations of a pipeline containing flowing fluid. Journal of Applied Mechanics., v. 19, n. 2, p. 205-208, 1952.

IBRAHIM, R. A. Overview of mechanics of pipes conveying fluids-part i: Fundamental studies. Journal of Pressure Vessel Technology, ASME International, v. 132, n. 3, may 2010 .

IBRAHIM, R. A. Mechanics of pipes conveying fluids-part II: Applications and fluidelastic problems. Journal of Pressure Vessel Technology, ASME International, v. 133, n. 2, feb 2011.

IRSCHIK, H.; HOLL, H. J. The equations of lagrange written for a non-material volume. Acta Mechanica, v. 153, n. 3-4, p. 231-248, sep 2002.

JENSEN, J. S. Articulated pipes conveying fluid pulsating with high frequency. Nonlinear Dynamics, Springer Nature, v. 19, n. 2, p. 173-193, 1999.

JIN, J.; SONG, Z. Parametric resonances of supported pipes conveying pulsating fluid. Journal of Fluids and Structures, v. 20, n. 6, p. 763-783, aug 2005.

KHEIRI, M.; PAÏDOUSSIS, M.; POZO, G. C. D.; AMABILI, M. Dynamics of a pipe conveying fluid flexibly restrained at the ends. Journal of Fluids and Structures, Elsevier BV, v. 49, p. 360-385, aug 2014.

KHEIRI, M.; PAÏDOUSSIS, M. P. On the use of generalized hamilton's principle for the derivation of the equation of motion of a pipe conveying fluid. Journal of Fluids and Structures, v. 50, p. 18-24, 2014.

KOO, B.; KIM, M.; RANDALL, R. Mathieu instability of a spar platform with mooring and risers. Ocean Engineering, Elsevier BV, v. 31, n. 17-18, p. 2175-2208, dec 2004.

LEE, S.; CHUNG, J. New non-linear modelling for vibration analysis of a straight pipe conveying fluid. Journal of Sound and Vibration, Elsevier BV, v. 254, n. 2, p. 313-325, jul 2002.

MAZZILLI, C. E. N.; ANDRE, J. C.; BUCALEM, M. L.; CIFU, S. Lições em Mecânica das Estruturas: Dinâmica. [S.l.]: Edgard Blucher LTDA, 2016.

MCIVER, D. B. Hamilton's principle for systems of changing mass. Journal of Engineering Mathematics, v. 7, n. 3, p. 249-261, jul 1973.

MEIROVITCH, L. Methods of Analytical Dynamics. [S.l.]: Dover Publications, 2003.

MENG, S.; SONG, S.; CHE, C.; ZHANG, W. Internal flow effect on the parametric instability of deepwater drilling risers. Ocean Engineering, v. 149, p. 305-312, feb 2018.

NAYFEH, A. H. Problems in Perturbation. [S.l.: s.n.], 1985.

NAYFEH, A. H.; BALACHANDRAN, B. Applied Nonlinear Dynamics. [S.l.]: Wiley-VCH Verlag GmbH, 1995. 
NAYFEH, A. H.; MOOK, D. T. Nonlinear oscillations. [S.l.]: John Wiley \& Sons, 1979.

ORSINO, R. M. M. A contribution on modeling methodologies for multibody systems. Tese (Doutorado), 2016.

ORSINO, R. M. M.; GIULIO, R. L. Lumped-parameter modular modeling of a pipe conveying fluid. In: DINAME. [S.l.: s.n.], 2019.

ORSINO, R. M. M.; PESCE, C. P. Reduced order modeling of a cantilevered pipe conveying fluid applying a modular methodology. International Journal of Non-Linear Mechanics, v. 103, p. 1-11, jul 2018.

PAÏDOUSSIS, M. P. Fluid-Structure Interactions - slender structures and axial flow Volume 1. [S.1.]: Academic Press, 1998.

PANDA, L.; KAR, R. Nonlinear dynamics of a pipe conveying pulsating fluid with combination, principal parametric and internal resonances. Journal of Sound and Vibration, v. 309, n. 3-5, p. 375-406, jan 2008.

PATEL, M. H.; PARK, H. I. Dynamics of tension leg platform tethers at low tension. Part I - Mathieu stability at large parameters. Marine Structures, v. 4, p. 257-273, 1991.

PESCE, C. P. Mecânica de cabos e tubos submersos lançados em catenária: Uma abordagem analítica experimental. Tese de livre docência, 1997.

RAINEY, R. C. T. The dynamics of tethered platforms. Transactions of the Royal Institution of Naval Architects, 1977.

RAND, R. H. Lecture Notes on Nonlinear Vibrations. [S.l.: s.n.], 2012.

ROUSSELET, J.; HERRMANN, G. Influence of nonlinearities on the behavior of parametrically excited articulated pipes conveying fluid. Transactions of the Canadian Society for Mechanical Engineering, Canadian Science Publishing, v. 5, n. 1, p. 31-38, mar 1978.

SADEGHI, Y. M.; PAÏDOUSSIS, M. Nonlinear dynamics of extensible fluid-conveying pipes, supported at both ends. Journal of Fluids and Structures, Elsevier BV, v. 25, n. 3, p. 535-543, apr 2009.

SIMOS, A. N.; PESCE, C. P. Mathieu stability in the dynamics of TLP's tethers considering variable tension along the length. In: Transactions on the Built Enviroment, vol. 29. Originally presented at Offshore Brazil 1997. [S.1.: s.n.], 1997.

TANG, D.; DOWELL, E. Chaotic oscillations of a cantilevered pipe conveying fluid. Journal of Fluids and Structures, Elsevier BV, v. 2, n. 3, p. 263-283, may 1988.

THOMPSON, J. Instabilities and Catastrophes in Science And Engineering. [S.1.]: John Wiley \& Sons Ltd., 1982.

VERNIZZI, G. J.; FRANZINI, G. R.; LENCI, S. Reduced-order models for the analysis of a vertical rod under parametric excitation. International Journal of Mechanical Sciences, Elsevier BV, v. 163, p. 105122, nov 2019. 
WEAVER, D.; PAIDOUSSIS, M. On collapse and flutter phenomena in thin tubes conveying fluid. Journal of Sound and Vibration, Elsevier BV, v. 50, n. 1, p. 117-132, jan 1977. 


\section{A Modelo 1 grau de liberdade}

O presente apêndice visa discutir o problema de um tubo rígido ejetando fluido e sob movimento vertical prescrito ao suporte ao qual ele é articulado.

\section{A.1 Determinação das equações de movimento}

Nesta seção será mostrado com detalhes como foi encontrada a equação de movimento não linear e linearizada para o modelo da Figura 38.

Figura 38 - Modelo 1 grau de liberdade.
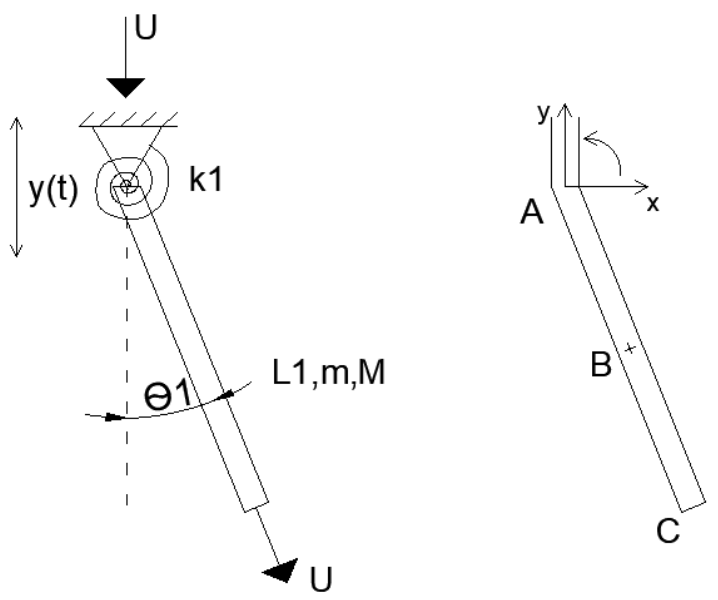

Na Figura 38 pode ser visto também os pontos auxiliares A,B e C. Esses pontos permitirão calcular as velocidades nos trechos desejados. A coordenada de cada ponto pode ser vista na Equação A.1. O vetor que representa a parcela da velocidade angular está mostrado na Equação A.2. Todos os vetores foram tomados a partir do eixo de coordenadas posicionado junto à posição inicial do ponto A, isto é, antes da imposição do movimento.

$$
\begin{array}{r}
A=(0 ;-y(t) ; 0) \\
B=\left(\frac{L_{1}}{2} \sin \theta_{1} ;-\frac{L_{1}}{2} \cos \theta_{1}-y(t) ; 0\right) \\
C=\left(\sin \theta_{1} ;-\cos \theta_{1}-y(t) ; 0\right) \\
\mathbf{w}_{1}=\left[0 ; 0 ; \dot{\theta}_{1}\right]
\end{array}
$$

A partir destas informações consegue-se chegar nos vetores de velocidade (ver Equação A.3) 


$$
\begin{array}{r}
\mathbf{v}_{A}=[0 ; \dot{y}(t) ; 0] \\
\mathbf{v}_{B}=\mathbf{v}_{A}+\mathbf{w}_{1} \times(B-A) \\
\mathbf{v}_{C}=\mathbf{v}_{A}+\mathbf{w}_{1} \times(C-A)
\end{array}
$$

Encontrando os valores das velocidades e utilizando a equação de energia cinética de um corpo rígido, pode-se encontrar as expressões para energia cinética do tubo $T_{1}$ e do fluido $T_{f l}$. Essas parcelas estão descritas pela Equação A.4).

$$
\begin{gathered}
T_{1}=\frac{1}{2} m L_{1} \mathbf{v}_{B} \cdot \mathbf{v}_{B}+\frac{1}{2} I_{B} \mathbf{w}_{1} \cdot \mathbf{w}_{1} \\
T_{f 1}=\frac{1}{2} M L_{1} \mathbf{v}_{B} \cdot \mathbf{v}_{B}+\frac{1}{2} I_{f B} \cdot \mathbf{w}_{1} \mathbf{w}_{1}
\end{gathered}
$$

sendo os termos referentes ao momento de inércia de massa são $I_{B}=\frac{1}{12} m L_{1}^{3}$ e $I_{f B}=\frac{1}{12} M L_{1}^{3}$. A energia cinética total $T_{\text {Total }}$ do sistema é a soma das parcelas da Equação A.4.

$$
\begin{gathered}
T_{\text {Total }}=T_{1}+T_{f 1} \\
T_{\text {Total }}=\frac{1}{8} L_{1}^{3}(m+M) \dot{\theta}_{1}^{2}+\frac{1}{24} L_{1}^{3}(m+M) \dot{\theta}_{1}^{2}+\frac{1}{2} L_{1}(m+M) \dot{y}^{2}+\frac{1}{2} L_{1} M U^{2} \\
-\frac{1}{2} L_{1}^{2}(m+M) \sin \theta_{1} \dot{\theta}_{1} \dot{y}+L_{1} M U \cos \theta_{1} \dot{y}
\end{gathered}
$$

As parcelas referentes à energia potencial podem ser obtidas da com o uso da Equação A.7).

$$
\begin{array}{r}
E_{1}=L_{1} m g\left(\frac{L_{1}}{2}-\frac{L_{1}}{2} \cos \theta_{1}-y\right) \\
E_{f 1}=L_{1} M g\left(\frac{L_{1}}{2}-\frac{L_{1}}{2} \cos \theta_{1}-y\right) \\
E_{k 1}=\frac{1}{2} k_{1} \theta_{1}^{2}
\end{array}
$$

A energia potencial total pode ser vista na Equação A.9.

$$
\begin{gathered}
E_{\text {Total }}=E_{1}+E_{f 1}+E_{k 1} \\
E_{\text {Total }}=L_{1}(m+M) g\left(\frac{L_{1}}{2}-\frac{L_{1}}{2} \cos \theta_{1}-y\right)+\frac{1}{2} k_{1} \theta_{1}^{2}
\end{gathered}
$$


Com as informações adquiridas até este ponto, é possível calcular as equações de movimento utilizando o principio estendido de Hamilton ou as equações de Euler-Lagrange. Neste trabalho foi optado pelas equações de Euler-Lagrange devido a maior simplicidade de implementação no software Wolfram Mathematica ${ }^{\circledR}$.

A equação de Euler-Lagrange é apresentada Equação A.10. Nessa equação já foi incluída a parcela do efeito de ponta mostrada anteriormente na Equação 3.9. O termo $L$ é conhecido como Lagrangeano e é definido como a diferença entre as energias cinética e potencial $\left(L=T_{\text {Total }}-E_{\text {Total }}\right)$.

$$
\frac{d}{d t}\left(\frac{\partial L}{\partial \dot{\theta}_{1}}\right)-\frac{\partial L}{\partial \theta_{1}}+M U\left[\dot{\mathbf{r}}_{L}+U \mathbf{n}_{L}\right] \cdot \frac{\partial \mathbf{r}_{L}}{\partial \theta_{1}}=0
$$

O vetor posição $\mathbf{r}_{L}$ é obtido em relação a um sistema de referência posicionado na extremidade livre do conjunto quando este está em repouso. Para melhor compreensão de como este vetor foi escrito basta observar a Figura 39.

Figura 39 - Obtenção do vetor posição $\mathbf{r}_{L}$.

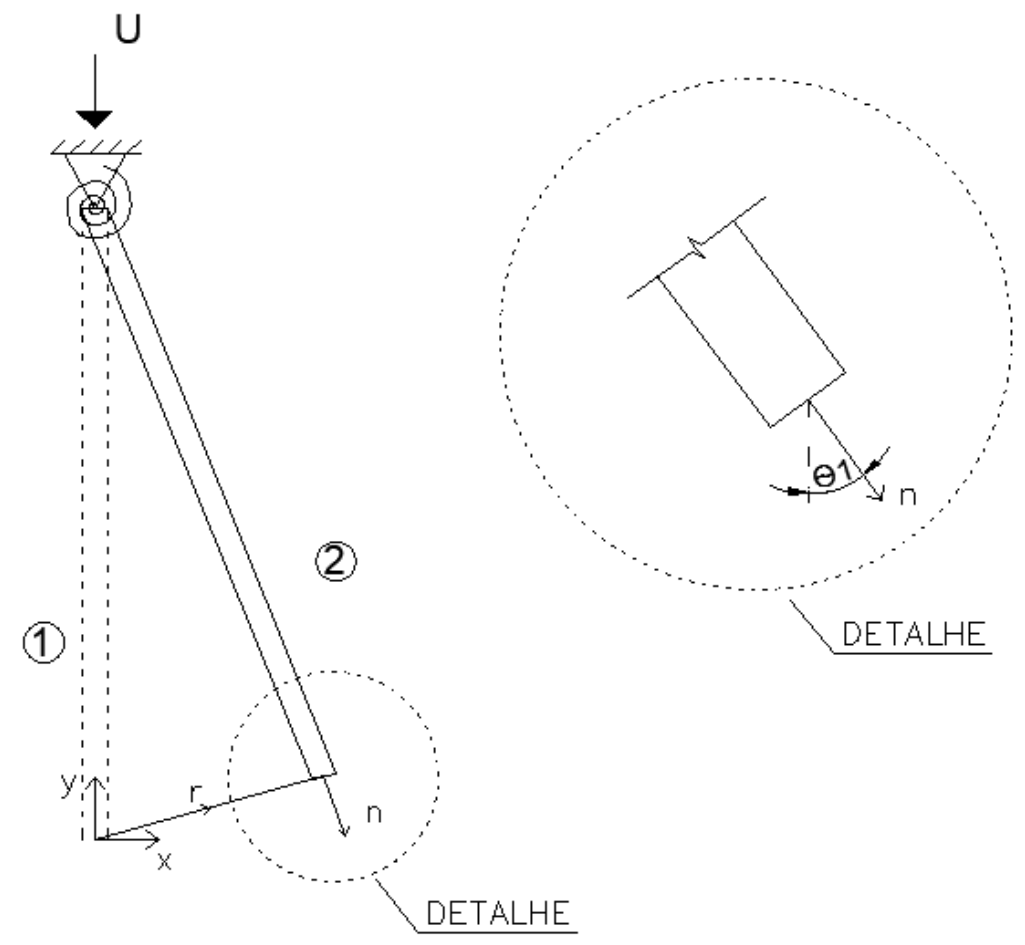

$\mathrm{O}$ vetor $\mathbf{r}_{L}$ pode ser escrito da seguinte forma,

$$
\mathbf{r}_{L}=\left[L_{1} \sin \theta_{1} ; L_{1}-L_{1} \cos \theta_{1}-y ; 0\right]
$$

O vetor velocidade $\dot{\mathbf{r}}_{L}$ é encontrado a partir da derivada do vetor posição $\mathbf{r}_{L} \mathrm{em}$ relação ao tempo e o versor direcional $\mathbf{n}$ é normal a posição da extremidade livre do tubo 
e pode ser visto na Equação A.12.

$$
\mathbf{n}=\left[\sin \theta_{1} ;-\cos \theta_{1} ; 0\right]
$$

Utilizando a Equação A.10 e todos os conceitos discutidos chega-se a equação não linear do movimento. Cabe lembrar que a excitação de suporte é suposta harmônica e monocromática, ou seja, da forma $y=A \cos (\omega t)$. Assim, é trivial notar que $\ddot{y}=$ $-A \omega^{2} \cos (\omega t)$.

$$
\frac{1}{3} L_{1}^{3}(M+m) \ddot{\theta}_{1}+\left(M U L_{1}^{2}+c_{1}\right) \dot{\theta}_{1}+k_{1} \theta_{1}+\frac{1}{2} L_{1}^{2}(M+m)(g-\ddot{y}) \sin \theta_{1}=0
$$

A Equação A.12 pode ser linearizada para análises em regime subcrítico. Neste caso utilizando expansões em séries de potência, para pequenos ângulos em torno da posição vertical $\theta_{1}=0$, considera-se $\sin \theta_{1}=\theta_{1}$. Desta forma a equação linearizada pode ser vista a seguir.

$$
\frac{1}{3} L_{1}^{3}(M+m) \ddot{\theta}_{1}+\left(M U L_{1}^{2}+c_{1}\right) \dot{\theta}_{1}+\left(k_{1}+\frac{1}{2} L_{1}^{2}(M+m)(g-\ddot{y})\right) \theta_{1}=0
$$

Uma forma de generalizar as análises é adimensionalizar a equação de movimento. Foram escolhidos os seguintes adimensionais (ver Equação A.15) respeitando a relação $\tau=\omega t$, essa escolha foi feita de modo a recuperar a Equação 2.19.

$$
\begin{gathered}
\beta=\frac{M}{(M+m)}, \epsilon=\frac{A}{L_{1}}, \delta=\frac{g}{\omega^{2} L_{1}}, K_{1}=\frac{k_{1} L_{1}}{E I}, \psi=\sqrt{\frac{E I}{g L_{1}^{3}(m+M)}} \\
u=U L_{1} \sqrt{\frac{M}{E I}}
\end{gathered}
$$

A equação de movimento linearizada escrita em forma adimensional é, então, dada por:

$$
\frac{1}{3} \theta_{1}^{\prime \prime}+\left(u \beta^{1 / 2} \delta^{1 / 2} \psi+C_{1}\right) \theta_{1}^{\prime}+\left(\delta \psi^{2} K_{1}+\frac{1}{2} \delta+\frac{1}{2} \epsilon \cos (\tau)\right) \theta_{1}=0
$$

$\operatorname{com} \theta^{\prime}=\partial \theta / \partial \tau$

Na Equação A.16, $C_{1}$ é a parcela adimensionalizada do amortecimento proporcional. Seu formato adimesional pode ser visto na Equação A.17.

$$
C_{1}=\left(\delta \psi^{2} K_{1}+\frac{1}{2} \delta\right) a_{1}+\frac{1}{3} a_{0}
$$




\section{A.2 Resultados}

Para a resolução de modelo de um grau de liberdade foi utilizada a Equação A.16 e os adimensionais definidos na Equação A.15. Para o problema de 1 grau de liberdade quando a velocidade do escoamento interno e o coeficiente de rigidez da mola são iguais a zero, a equação de movimento assume a forma da equação de Mathieu e o resultado recai no digrama de Strutt apresentado em Nayfeh e Mook (1979).

A Figura 40 foi gerada com base na ferramenta aqui preparada considerando ${ }^{1}$ $u=0.0, K_{1}=0.0$ e $C_{1}=0.0$, já a Figura 41 foi extraída de Nayfeh e Mook (1979). Como esperado, o resultado recai no problema clássico encontrado na literatura.

Figura 40 - Diagrama de instabilidade para o modelo de 1 grau de liberdade considerando $u=0$ e $K_{1}=0$.

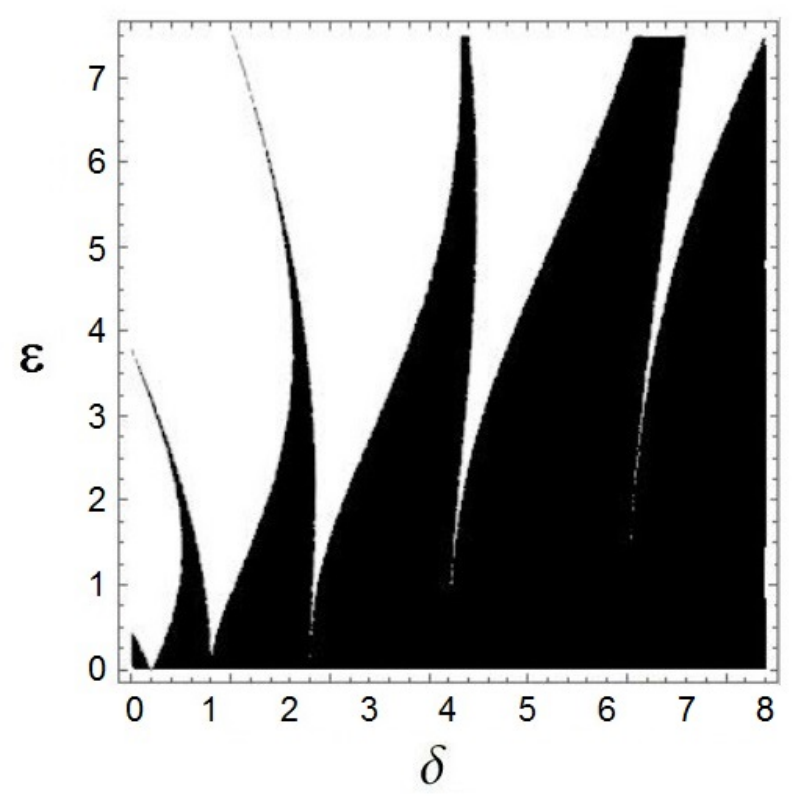

Comparando o resultado obtido com aquele disponível na literatura garante confiabilidade à rotina desenvolvida neste trabalho, de sorte que ela será aplicada às demais condições de velocidade de escoamento interno e de parâmetros estruturais.

1 Nos diagramas apresentados a região preenchida com a cor preta representa a zona de estabilidade, enquanto a região preenchida com a coloração branca a zona de instabilidade. 
Figura 41 - Diagrama de Strutt para equação de Mathieu

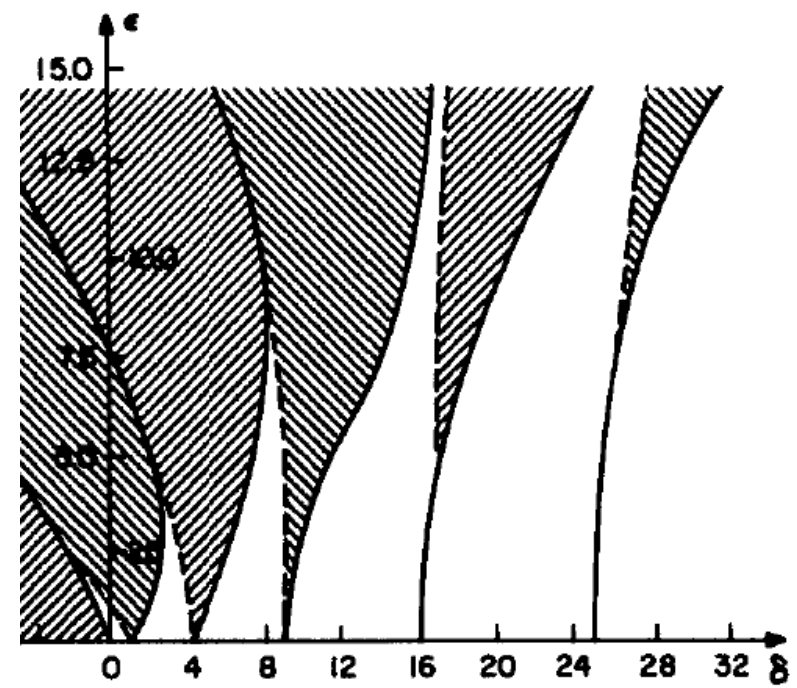

Fonte: Nayfeh e Mook (1979). 